Authors are encouraged to submit new papers to INFORMS journals by means of a style file template, which includes the journal title. However, use of a template does not certify that the paper has been accepted for publication in the named journal. INFORMS journal templates are for the exclusive purpose of submitting to an INFORMS journal and should not be used to distribute the papers in print or online or to submit the papers to another publication.

\title{
$\epsilon$-Strong Simulation of Fractional Brownian Motion and Related Stochastic Differential Equations
}

\author{
Yi Chen \\ Northwestern University, yichen2016@u.northwestern.edu, \\ Jing Dong \\ Columbia University, jing.dong@gsb.columbia.edu, \\ $\mathrm{Hao} \mathrm{Ni}$ \\ University College London, h.ni@ucl.ac.uk,
}

Consider a fractional Brownian Motion (fBM) $B^{H}=\left\{B^{H}(t): t \in[0,1]\right\}$ with Hurst index $H \in(0,1)$. We construct a probability space supporting both $B^{H}$ and a fully simulatable process $\hat{B}_{\epsilon}^{H}$ such that

$$
\sup _{t \in[0,1]}\left|B^{H}(t)-\hat{B}_{\epsilon}^{H}(t)\right| \leq \epsilon
$$

with probability one for any user specified error bound $\epsilon>0$. When $H>1 / 2$, we further enhance our error guarantee to the $\alpha$-Hölder norm for any $\alpha \in(1 / 2, H)$. This enables us to extend our algorithm to the simulation of fBM driven stochastic differential equations $Y=\{Y(t): t \in[0,1]\}$. Under mild regularity conditions on the drift and diffusion coefficients of $Y$, we construct a probability space supporting both $Y$ and a fully simulatable process $\hat{Y}_{\epsilon}$ such that

$$
\sup _{t \in[0,1]}\left|Y(t)-\hat{Y}_{\epsilon}(t)\right| \leq \epsilon
$$

with probability one. Our algorithms enjoy the tolerance-enforcement feature, under which the error bounds can be updated sequentially in an efficient way. Thus, the algorithms can be readily combined with other advanced simulation techniques to estimate the expectations of functionals of fBMs efficiently.

Key words: fractional Brownian motion, Stochastic differential equation, Monte Carlo Simulation

1. Introduction The fractional Brownian motion (fBM) of Hurst parameter $H \in(0,1)$, $\left\{B^{H}(t): t \geq 0\right\}$, is a centered real-valued Gaussian process with covariance function

$$
r(s, t):=\mathbb{E}\left[B^{H}(s) B^{H}(t)\right]=\frac{1}{2}\left(s^{2 H}+t^{2 H}-|s-t|^{2 H}\right) .
$$

When $H=1 / 2$, fBM is a Brownian motion (BM), which has independent increments. When $H<1 / 2$, the increments of fBM are negatively correlated. In contrast, when $H>1 / 2$, fBM has positively correlated increments and displays long-range dependence. Generally, fBM can be viewed as an extension of BM. As it allows the increments to be correlated, it has been applied in communications engineering (Norros, 1995), biology, and physics (Höfling and Franosch, 2013). See also Laskin (2000) and Nualart (2006) for applications in finance and turbulence. However, due to 
the correlated increments (lack of Markovian structure), very few closed-form expressions are known for performance measures related to functionals of fBMs. In this context, simulation-based numerical method has been a powerful tool to conduct performance analysis for fBM driven processes.

In this paper, we develop a new class of algorithms to construct paths of fBM and fBM driven stochastic differential equations (SDEs) with strong error guarantees. In particular, the algorithm allows us to construct a probability space supporting both a fBM and a fully simulatable path $\hat{B}_{\epsilon}^{H}$, such that

$$
\sup _{0 \leq t \leq 1}\left|B^{H}(t)-\hat{B}_{\epsilon}^{H}(t)\right| \leq \epsilon \text { w.p. } 1 .
$$

Moreover, when $H>1 / 2$, for any $\alpha \in(1 / 2, H)$, we can further construct a fully simulatable path $\hat{B}_{\epsilon}^{H}$, such that

$$
\sup _{0<s \leq t \leq 1} \frac{\left|B^{H}(t)-\hat{B}_{\epsilon}^{H}(t)-\left(B^{H}(s)-\hat{B}_{\epsilon}^{H}(s)\right)\right|}{|t-s|^{\alpha}} \leq \epsilon \text { w.p. } 1 .
$$

For $H>1 / 2$, the control of the $\alpha$-Hölder norm allows us to use the rough path theory to construct a probability space supporting both a fBM-driven SDE

$$
d Y(t)=\mu(Y(t)) d t+\sigma(Y(t)) d B^{H}(t),
$$

and a sequence of fully simulatable path $\hat{Y}_{\epsilon}$, such that

$$
\sup _{0 \leq t \leq 1}\left|Y(t)-\hat{Y}_{\epsilon}(t)\right| \leq \epsilon \text { w.p. } 1 .
$$

In terms of the computational complexity, our algorithm achieves the near optimal complexity. Specifically, for fBM, to achieve an $\epsilon$ error bound, the expected computational cost is $O\left(\epsilon^{-1 /(H-\delta)}\right)$ for any $\delta \in(0, H)$. When $H>1 / 2$, for fBM driven SDEs, under suitable regularity conditions on the drift and diffusion terms, to achieve an $\epsilon$ error bound, the expected computation cost is $O\left(\epsilon^{-1 /(2 \alpha-1)}\right)$ for any $\alpha \in(1 / 2, H)$.

In addition to the strong error guarantee, the framework we developed also enjoys the toleranceenforcement feature. Specifically, for any sequence $0<\epsilon_{n}<\epsilon_{n-1}<\cdots<\epsilon_{1}$, we can adaptively simulate $B_{\epsilon_{n}}^{H}$ conditional on $B_{\epsilon_{1}}^{H}, \ldots, B_{\epsilon_{n-1}}^{H}$. This feature allows us to easily combine our procedure with other advanced simulation/randomization techniques to remove estimation bias (see, for example, Rhee and Glynn (2012); Beskos et al. (2012); Blanchet and Chen (2015)).

The simulation framework we developed in this paper contribute to recent development in $\epsilon$-strong simulation. Beskos et al. (2012) is among the first to introduce the concept $\epsilon$-strong simulation. There, the authors develop an $\epsilon$-strong simulation algorithm for Brownian motion (BM). Later, Blanchet and Chen (2015) and Blanchet et al. (2017) extend the framework to reflected Brownian motion and multidimensional stochastic differential equations respectively. Our paper is the first work that develops $\epsilon$-strong simulation algorithm to $\mathrm{fBM}$ and fBM driven SDEs. The extension from processes related to $\mathrm{BM}$ to $\mathrm{fBM}$ is highly nontrivial, and involves the development of new theoretical results and simulation techniques. To see where the fundamental differences are from, note that fBMs with $H \neq 1 / 2$ no longer have independent increments.

In the process of developing the simulation algorithms, we establish several important properties of fBM and fBM driven SDEs. Most noticeably, We provide bounds for the convergence rate of midpoint displacement approximation of fBM. Although midpoint displacement and variants of it (e.g., random midpoint displacement) has been applied to approximate fBM (Lau et al., 1995; Norros et al., 1999), our paper is the first to rigorously study the convergence rate of this approximation scheme. Our analysis builds on the decay rate of the conditional mean and covariance 
of fBM, and can be applied to more general Gaussian processes beyond fBM. The convergence results may be of independent interests. For example, it enables us to combine the midpoint displacement approximation of fBM with Multilevel Monte Carlo (MLMC) for efficient estimation of expectations (Giles, 2008). In MLMC, the key challenges lie in constructing a suitable coupling for the differences in the telescoping sum and bounding the variance of the coupled differences. The midpoint displacement construction provides a natural coupling and our convergence rate analysis provides closed-form bounds for the corresponding variances.

In terms of the algorithmic development, our method builds on the idea of record-breakers. This idea was first introduced in Blanchet and Sigman (2011) for exact sampling of stochastic perpetuities. Later, similar ideas have been applied to exact simulation of queueing models in steady-state (Blanchet and Dong, 2015; Blanchet and Chen, 2015), max-stable processes and related random fields (Liu et al., 2019), etc. There are two key challenges in applying this idea. The first is to define a proper sequence of record-breaking events, which relies on a good understanding of the convergence rate for various approximations of the underlying processes. We overcome this challenge by establishing strong convergence results for the midpoint displacement construction of fBM. The second challenge is to find the last time the 'record' is broken. We overcome this challenge by applying and extending ideas from rare-event simulation.

The rest of the paper is organized as follows. We conclude this section with a brief review of the literature. We introduce the basic idea of our algorithmic development in Section 2. We conduct convergence rate analysis of the midpoint displacement decomposition of fBM in Section 3. This provides the theoretical basis for the construction of our algorithm. The results and proof techniques can be of interests to the analysis of other Gaussian processes. The details of the actual simulation algorithm for fBM are provided in Section 4. In Section 5, we extend the algorithm to SDEs driven by $\mathrm{fBM}$ with Hurst index $H>1 / 2$. Lastly, in Section 6 we conduct some numerical experiments as a sanity check and provide some comments about actual implementations of our algorithm. Several detailed proofs are deferred until the Appendix. We also provide more details about applications of our results to MLMC and unbiased estimation in Appendix D.

Throughout the paper, for $\boldsymbol{v}$, which can be a vector, a matrix, or a tensor, and we use $\|\boldsymbol{v}\|$ to denote the maximum of the absolute value of its entries. When $\boldsymbol{v}$ is a vector, we use $(\boldsymbol{v})_{i}$ to denote its $i$-th component. By default, all the vectors are column vectors. We denote

$$
\|\boldsymbol{u}\|_{\infty}:=\sup _{0 \leq t \leq 1}\|\boldsymbol{u}(t)\| \text { and }\|\boldsymbol{u}\|_{\alpha}:=\sup _{0 \leq s<t \leq 1} \frac{\|\boldsymbol{u}(s)-\boldsymbol{u}(t)\|}{|s-t|^{\alpha}}
$$

as the supremum norm and $\alpha$-Hölder norm of a generic function $\boldsymbol{u}$ on $[0,1]$ respectively. Let $\mathcal{C}([0,1])$ be the space of continuous functions over $[0,1]$ equipped with uniform norm $\|\cdot\|_{\infty}$. We also write $\mathbb{Z}^{+}$as the set of positive integers and $\mathbb{R}$ as the set of real numbers. For two nonnegative functions $f$ and $g$ defined on $\mathbb{Z}^{+}$, we define $f(n)=O(g(n))$ if there exists $M>0$ such that $|f(n)| \leq M g(n)$. We also define $f(n)=\Theta(g(n))$ if there exists $0<L<M<\infty$ such that $L g(n) \leq f(n) \leq M g(n)$.

1.1. Literature review Our work is closely related to the line of research on simulation of fBM. Existing methods can be divided into two categories, exact method and approximation method (see Dieker (2004) for a detailed survey).

The exact methods aim to generate the fBM at a fixed finite set of time points from the exact distribution. To carry out this task efficiently is highly nontrivial, due to the correlation structure of fBM. Naive implementation using the Cholesky decomposition has a complexity of $O\left(n^{3}\right)$ for $n$ points in general. More efficient methods have been developed in the literature, mostly of them utilizing the stationarity of the process (see, for example, Levinson (1946); Durbin (1960); Davies and Harte (1987)). Among them, the circulant embedding method is able the reduce the complexity to $O(n \log n)$ (Dietrich and Newsam, 1997; Wood and Chan, 1994). Our method aims to recover 
the whole fBM path, but it builds on being able to generate the fBM at finite set of points exactly. However, as our algorithm relies on sequentially updating the set of points (at finer and finer dyadic levels), we can no longer utilize the stationarity structure.

The approximation methods aim to generate approximations of the fBM sample path. Some approximation methods build on special representations of $\mathrm{fBM}$, infinite series representations in particular (see, for example, Abry and Sellan (1996); Dieker and Mandjes (2003); Ayache and Taqqu (2003)). Our method also builds a suitable infinite series decomposition of the fBM based on midpoint displacement. However, instead of applying a deterministic truncation level, our truncation level is adapted to the sample path, which is random. Our work extends this line of literature by achieving a stronger error guarantee. We also note that like a lot of the approximation methods developed in the literature, our algorithm is for pre-specified fixed time horizons.

2. Basic idea We start by introducing the basic idea of our algorithmic development. Recall that fBM $B^{H}$ is a centered real-valued Gaussian process with the covariance function given in (1). By Kolmogorov continuity theorem, fBM has a continuous modification. Moreover, for any $\alpha \in(0, H)$ and $T>0$, this modification is $\alpha$-Hölder continuous on $[0, T]$. In this paper, we refer to such a modification as the fBM, and focus on a finite time interval $[0,1]$ with $B^{H}(0)=0$.

The algorithmic development consists of two steps. First, we identify an infinite series expansion of fBM, i.e.,

$$
B^{H}(t)=\sum_{k=0}^{\infty} \Lambda_{k}(t) W_{k},
$$

where $\Lambda_{k}$ 's are a sequence of basis functions, and $W_{k}$ 's are the random coefficients. We then develop an algorithm to truncate the infinite sum up to a finite but random level, $K$, so that the error induced by the truncated terms is suitably controlled, e.g.

$$
\sup _{0 \leq t \leq 1}\left|\sum_{k=0}^{K} \Lambda_{k}(t) W_{k}-B^{H}(t)\right| \leq \epsilon \text { w.p. } 1 .
$$

Many infinite series expansions of fBM are developed in the literature: Some are based on wavelet decomposition (multi-resolution framework) (see, for example, (Meyer et al., 1999)); Others are based on Karhunen-Loéve type of expansion (spectral theory) (see, for example, Dzhaparidze and Zanten (2004)). Our consideration here is twofold. First, the infinite series expansion needs to converge fast in an almost sure sense under uniform norm or even the $\alpha$-Hölder norm. Second, the corresponding simulation algorithm can be implemented efficiently. To fulfill these two requirements, we choose Lévy's midpoint displacement technique, which corresponds to the wavelet decomposition using the Haar wavelets. The challenge here is that when $H \neq 1 / 2$, the coefficients are correlated. We shall provide more analysis about the random coefficient terms in Section 3.

The actual midpoint displacement construction goes as follows. Let $D_{n}$ be the dyadic discretization of order $n$ and $\Delta_{n}$ be the mesh of the discretization. Specifically,

$$
D_{n}=\left\{t_{0}^{n}, t_{1}^{n}, \cdots, t_{2^{n}}^{n}\right\} \text {, where } t_{i}^{n}=i / 2^{n}, i=0,1, \cdots, 2^{n} \text {, and } \Delta_{n}=1 / 2^{n} \text {. }
$$

We use $\boldsymbol{B}_{n}^{H}=\left(B^{H}\left(t_{0}^{n}\right), \ldots, B^{H}\left(t_{2^{n}}^{n}\right)\right)^{\top}$ to denote the value of fBM at discretization level $n$. Given a realization of $\boldsymbol{B}_{n}^{H}$, we can construct a continuous path $B_{n}^{H}$ over time interval $[0,1]$ via linear interpolation and we call $B_{n}^{H}$ a dyadic discretization of fBM at level $n$. We notice that $B_{n}^{H}(t)=$ $B_{n-1}^{H}(t)$ for $t \in D_{n-1}$. At the augmented points $D_{n} \backslash D_{n-1}=\left\{t_{2 i+1}^{n}\right\}_{i=0, \cdots, 2^{n-1}-1}$, we have

$$
B_{n}^{H}\left(t_{2 i+1}^{n}\right)-B_{n-1}^{H}\left(t_{2 i+1}^{n}\right)=B^{H}\left(t_{2 i+1}^{n}\right)-\frac{1}{2}\left(B^{H}\left(t_{i}^{n-1}\right)+B^{H}\left(t_{i+1}^{n-1}\right)\right) .
$$


In Section 3, we show that the following infinite series representation is valid

$$
B^{H}(t)=\sum_{k=0}^{\infty}\left[B_{k}^{H}(t)-B_{k-1}^{H}(t)\right]
$$

i.e., $B_{n}^{H}$ converges to $B^{H}$ almost surely under the uniform norm (Theorem 2 ). Here, $B_{-1}^{H}(t)$ is a constant function with value zero. Note that when we truncate the infinite series at level $n$, we obtain $B_{n}^{H}$. We also establish the rate of convergence for $B_{n}^{H}$ (Theorem 3 and Theorem 4). In particular,

$$
\left\|B_{n}^{H}-B^{H}\right\|_{\infty}=O\left(2^{-(H-\delta) n}\right) \text { a.s. for any } \delta \in(0, H) .
$$

Note that when the $W_{k}$ 's in the series representation (2) are i.i.d. standard Gaussian random variables, Kühn and Linde (2002) show that

$$
\inf _{\left\{\Lambda_{k}\right\}_{k \geq 1}}\left\{\mathbb{E}\left[\sup _{t \in[0,1]}\left|\sum_{k=1}^{n} \Lambda_{k}(t) W_{k}-B^{H}(t)\right|^{2}\right]^{1 / 2}: B^{H}(t)=\sum_{k=1}^{\infty} \Lambda_{k}(t) W_{k} \text { a.s. }\right\}=\Theta\left(n^{-H}(\log n)^{1 / 2}\right) .
$$

i.e., the optimal rate of convergence, under the $L_{2}$ norm, is $\Theta\left(n^{-H}(\log n)^{1 / 2}\right)$. The midpoint displacement representation achieves almost the same rate of convergence as $\delta$ can be sent arbitrarily small. (Note that the $n$-th dyadic level involves $2^{n}$ time points.)

We next introduce the algorithmic development to truncate the infinite sum. Our goal here is to control the error of the infinite truncated terms. To achieve this, we adopt the strategy of "record-breakers". The general idea is to define a sequence of events called record-breakers, which satisfy the following two conditions

C1) The following event happens with probability one: beyond some random but finite level, there will be no more record-breakers;

C2) By knowing that there are no more record-breakers, the contribution of the infinite remaining terms are well under control.

Based on the convergence rate of $B_{n}^{H}$, we say that a record is broken at level $n$ if

$$
\left\|B_{n}^{H}-B_{n-1}^{H}\right\|_{\infty} \geq \rho \cdot 2^{-(H-\delta) n} .
$$

Here $\delta \in(0, H)$ and $\rho>0$ are hyper-parameters, which will be specified in Theorem 1 (Section 3). We denote $N$ as the level of the last record-breaker, i.e.,

$$
N=\sup \left\{n \geq 1:\left\|B_{n}^{H}-B_{n-1}^{H}\right\|_{\infty} \geq \rho \cdot 2^{-(H-\delta) n}\right\} .
$$

For C1), we show in Theorem 3 that $N$ has a finite moment generating function. For C2), we notice that for $n \geq N$, we have

$$
\left\|\sum_{k=n+1}^{\infty}\left[B_{k}^{H}-B_{k-1}^{H}\right]\right\|_{\infty} \leq \rho \cdot \sum_{k=n+1}^{\infty} 2^{-(H-\delta) k} .
$$

Thus, once we know the time of the last record-breaker $N$, to achieve a certain accuracy $\epsilon$, we just need to find

$$
N(\epsilon)=\min \left\{n: \rho \cdot \sum_{k=n+1}^{\infty} 2^{-(H-\delta) k}<\epsilon\right\}
$$


Then $\left\|B_{N(\epsilon) \vee N}^{H}-B^{H}\right\|_{\infty}<\epsilon$, where $N(\epsilon) \vee N$ denotes the maximum of $N(\epsilon)$ and $N$. The error bound is achieved in a path-by-path sense. In addition, we show in Theorem 4 that conditional on $N$, we also have an explicit upper bound for the $\alpha$-Hölder norm of $B^{H}$ in a path-by-path sense. This is important to develop the $\epsilon$-strong simulation algorithm for fBM driven SDEs as outlined in Section 5 .

The remaining task is to find the level of the last record-breaker $N$. This is challenging as $N$ is not a stopping time under the filtration generated by the value of the fBM at different dyadic levels. Our strategy is to find the record-breakers sequentially until we find the last one. Let $\tau_{k}$ to denote the level of the $k$-th record-breaker, i.e.

$$
\begin{aligned}
& \tau_{0}=0 \\
& \tau_{k}=\inf \left\{n \geq \tau_{k-1}+1:\left\|B_{n}^{H}-B_{n-1}^{H}\right\|_{\infty}>\rho \cdot 2^{-(H-\delta) n}\right\}, k \geq 1 .
\end{aligned}
$$

Then we have

$$
N=\sup \left\{\tau_{k}: \tau_{k}<\infty\right\} .
$$

Conditional on $B_{\tau_{k}}^{H}, \tau_{k}<\infty$, we first use a change-of-measure to generate a candidate $B_{\tau_{k+1}}^{H}$. We then apply an acceptance-rejection step with a properly defined likelihood ratio. If the path is accepted, we find $\tau_{k+1}$ and $B_{\tau_{k+1}}^{H}$; Otherwise, we claim that $\tau_{k}$ is the level of the last record-breaker, i.e. $N=\tau_{k}$. The details of the algorithmic developments are provided in Section 4.

3. Midpoint displacement of fBM In this section, we analyze the midpoint displacement construction of fBM. This provides the theoretical foundation for our algorithmic development. Specifically, we establish the validity of the infinite series expansion (3), and analyze its rate of convergence under both the uniform norm and the $\alpha$-Hölder norm.

Recall that at the augmented points $D_{k} \backslash D_{k-1}=\left\{t_{2 j+1}^{k}\right\}_{j=0,1, \cdots, 2^{k-1}-1}$, we have

$$
B_{k}^{H}\left(t_{2 j+1}^{k}\right)-B_{k-1}^{H}\left(t_{2 j+1}^{k}\right)=B^{H}\left(t_{2 j+1}^{k}\right)-\frac{1}{2}\left(B^{H}\left(t_{j}^{k-1}\right)+B^{H}\left(t_{j+1}^{k-1}\right)\right) .
$$

For notational convenience, we denote

$$
a_{j}^{k}:=B^{H}\left(t_{2 j+1}^{k}\right), \quad b_{j}^{k}:=\frac{1}{2}\left(B^{H}\left(t_{j}^{k-1}\right)+B^{H}\left(t_{j+1}^{k-1}\right)\right) .
$$

Then since $B_{k}^{H}(t)-B_{k-1}^{H}(t)$ is linear over intervals $\left[t_{2 j}^{k}, t_{2 j+1}^{k}\right]$ and $\left[t_{2 j+1}^{k}, t_{2 j+2}^{k}\right]$, we have

$$
\left\|B_{k}^{H}-B_{k-1}^{H}\right\|_{\infty}=\max _{0 \leq j \leq 2^{k-1}-1}\left|a_{j}^{k}-b_{j}^{k}\right| .
$$

We first establish the convergence rate for $\left\{\left\|B_{k}^{H}-B_{k-1}^{H}\right\|_{\infty}\right\}_{k \in \mathbb{Z}^{+}}$, which lays the foundation of subsequent results. We define

$$
\ell_{k}:=2^{-(H-\delta) k}, \text { for any fixed } \delta \in(0, H) .
$$

For any constant $\nu>0$, we denote by

$$
K(\nu)=\sup \left\{n \geq 1: 4 \sqrt{n}>\nu \cdot 2^{\delta n}\right\} .
$$

Then we have the following theorem establishing bounds for $\left\|B_{k}^{H}-B_{k-1}^{H}\right\|_{\infty}$.

TheOREm 1. For any constant $\nu>0$ and $\nu^{*}>0$, we have, for all $k>K(\nu)$,

$$
\mathbb{P}\left(\left\|B_{k}^{H}-B_{k-1}^{H}\right\|_{\infty} \geq \rho \ell_{k}\right)=\mathbb{P}\left(\max _{0 \leq j \leq 2^{k-1}-1}\left|a_{j}^{k}-b_{j}^{k}\right| \geq \rho \ell_{k}\right) \leq 2 \exp \left\{-\left(\nu^{*}\right)^{2} \cdot 2^{2 k \delta-2}\right\},
$$

where $\rho=2\left(\nu+\nu^{*}\right)$. 
Before we introduce the detailed proof of Theorem 1, we comment that $\delta$ and $\rho$ are parameters characterizing the record-breakers. We have some freedom in choosing these parameters, and there is a tradeoff involved. For larger $\rho$, the record-breakers are less likely to happen and it is relatively faster to find the last record-breaker. However, as a cost, we need to truncate at a higher level to achieve the desired accuracy. We provide more discussion about the choice of these parameters in practice in Section 6 .

Proof of Theorem 1. Note that for fBM with Hurst index $H \neq 1 / 2, a_{j}^{k}-b_{j}^{k}$ is not a centered Gaussian random variable conditioning on $\boldsymbol{B}_{k-1}^{H}$. In the following, we use

$$
c_{j}^{k}=\mathbb{E}\left[B^{H}\left(t_{2 j+1}^{k}\right) \mid \boldsymbol{B}_{k-1}^{H}\right], j=0,1, \cdots, 2^{k-1}-1,
$$

to denote the conditional expectation of fBM at the augmented points $D_{k} \backslash D_{k-1}$ given $\boldsymbol{B}_{k-1}^{H}$. Then we have

$$
\max _{0 \leq j \leq 2^{k-1}-1}\left|a_{j}^{k}-b_{j}^{k}\right| \leq \max _{0 \leq j \leq 2^{k-1}-1}\left|a_{j}^{k}-c_{j}^{k}\right|+\max _{0 \leq j \leq 2^{k-1}-1}\left|c_{j}^{k}-b_{j}^{k}\right| .
$$

The two terms in the right-hand side of inequality (7) correspond to the variance and bias. In what follows, we will establish bounds for each of them.

It is easy to see that

$$
\mathbb{P}\left(\max _{0 \leq j \leq 2^{k-1}-1}\left|a_{j}^{k}-b_{j}^{k}\right| \geq \rho \ell_{k}\right) \leq \underbrace{\mathbb{P}\left(\max _{0 \leq j \leq 2^{k-1}-1}\left|a_{j}^{k}-c_{j}^{k}\right|>\rho \ell_{k} / 2\right)}_{(V)}+\underbrace{\mathbb{P}\left(\max _{0 \leq j \leq 2^{k-1}-1}\left|c_{j}^{k}-b_{j}^{k}\right|>\rho \ell_{k} / 2\right)}_{(B)} .
$$

The rest of the proof is divided into two parts. We first establish a bound for (V), which corresponds to the variance. We then establish a bound for (B), which corresponds to the bias. In subsequent analysis, we need several auxiliary results which are summarized in Lemmas 1-3.

For $(\mathrm{V})$, we have

$$
\mathbb{P}\left(\max _{0 \leq j \leq 2^{k-1}-1}\left|a_{j}^{k}-c_{j}^{k}\right|>\rho \ell_{k} / 2\right)=\mathbb{E}\left[\mathbb{P}\left(\max _{0 \leq j \leq 2^{k-1}-1}\left|a_{j}^{k}-c_{j}^{k}\right|>\rho \ell_{k} / 2 \mid \boldsymbol{B}_{k-1}^{H}\right)\right] .
$$

In the following, we use $\mathbb{P}_{k-1}(\cdot)$ to denote $\mathbb{P}\left(\cdot \mid \boldsymbol{B}_{k-1}^{H}\right)$, which is the conditional probability given the values of $\boldsymbol{B}_{k-1}^{H}$. We also use $\mathbb{E}_{k-1}$ and $\mathbb{V}_{k-1}$ to denote corresponding conditional expectation and variance. Then under the probability measure $\mathbb{P}_{k-1}(\cdot), a_{j}^{k}$ is a Gaussian random variable with mean $c_{j}^{k}$ and variance $\sigma_{k j}^{2}:=\mathbb{V}_{k-1}\left(a_{j}^{k}\right)$. The following lemma upper bounds $\sigma_{k j}^{2}$ uniformly for all $j$ 's.

Lemma 1. For all $k \geq 1$ and $j=0,1, \cdots, 2^{k-1}-1$, we have

$$
\mathbb{V}_{k-1}\left(a_{j}^{k}\right)=\sigma_{k j}^{2} \leq 2 \cdot 2^{-2 k H} .
$$

Using Lemma 1 and Borell-TIS inequality, for any $u>0$, we obtain

$$
\mathbb{P}_{k-1}\left(\max _{0 \leq j \leq 2^{k-1}-1}\left|a_{j}^{k}-c_{j}^{k}\right|-\mathbb{E}_{k-1}\left[\max _{0 \leq j \leq 2^{k-1}-1}\left|a_{j}^{k}-c_{j}^{k}\right|\right]>u\right) \leq \exp \left\{-u^{2} \cdot 2^{2 k H-2}\right\} .
$$

In order to get rid of the expectation in inequality (10), we need the following lemma to upper bound the expectation.

Lemma 2. Let $X_{1}, X_{2}, \cdots, X_{n}$ be a sequence of (not necessarily independent) centered Gaussian random variables whose variances are uniformly bounded by $\sigma^{2}$. Then we have

$$
\mathbb{E}\left[\max _{1 \leq i \leq n}\left|X_{i}\right|\right] \leq 2 \sqrt{2 \log (2 n)} \cdot \sigma .
$$


By applying Lemma 1 and 2, we obtain that for any $\nu>0$, there exists a $K(\nu)$ such that for all $k \geq K(\nu)$,

$$
\mathbb{E}_{k-1}\left[\max _{0 \leq j \leq 2^{k-1}-1}\left|a_{j}^{k}-c_{j}^{k}\right|\right] \leq 4 \sqrt{k} \cdot 2^{-k H} \leq \nu \ell_{k} .
$$

For any constant $\nu^{*}>0$, by setting $u=\nu^{*} \ell_{k}$ in inequality (10) and using inequality (11), we obtain

$$
\mathbb{P}_{k-1}\left(\max _{0 \leq j \leq 2^{k-1}-1}\left|a_{j}^{k}-c_{j}^{k}\right|>\left(\nu+\nu^{*}\right) \ell_{k}\right) \leq \exp \left\{-\left(\nu^{*}\right)^{2} \cdot 2^{2 k \delta-2}\right\} .
$$

Then based on (9), for the unconditional probability, we have

$$
\mathbb{P}\left(\max _{0 \leq j \leq 2^{k-1}-1}\left|a_{j}^{k}-c_{j}^{k}\right|>\left(\nu+\nu^{*}\right) \ell_{k}\right) \leq \exp \left\{-\left(\nu^{*}\right)^{2} \cdot 2^{2 k \delta-2}\right\} .
$$

Now, we turn to (B). In contrast to the previous proof where we deal with the conditional probability $\mathbb{P}_{k-1}(\cdot)$ first, in this part, we consider the unconditional probability $\mathbb{P}(\cdot)$ directly. In the following, to simplify notations, let $\boldsymbol{b}_{k}=\left(b_{0}^{k}, \cdots, b_{2^{k-1}-1}^{k}\right)^{\top}$ and $\boldsymbol{c}_{k}=\left(c_{0}^{k}, \cdots, c_{2^{k-1}-1}^{k}\right)^{\top}$. Then by definition, we have

$$
\boldsymbol{b}_{k}=\boldsymbol{M}_{k-1} \boldsymbol{B}_{k-1}^{H}, \text { where } \boldsymbol{M}_{k-1}=\left[\begin{array}{ccccc}
1 / 2 & 1 / 2 & 0 & \cdots & 0 \\
0 & 1 / 2 & 1 / 2 & \cdots & 0 \\
\cdots & \cdots & \cdots & \cdots & \cdots \\
0 & 0 & 0 & \cdots & 1 / 2
\end{array}\right]_{2^{k-1} \times\left(2^{k-1}+1\right)}
$$

Based on the conditional distribution of multivariate Gaussian random vector and the covariance function of fBM, we have $\boldsymbol{c}_{k}=\boldsymbol{N}_{k-1} \boldsymbol{B}_{k-1}^{H}$, where

$$
\boldsymbol{N}_{k-1}=\boldsymbol{\Sigma}_{12}^{(k-1)} \cdot\left[\boldsymbol{\Sigma}_{22}^{(k-1)}\right]^{-1}
$$

and $[\cdot]^{-1}$ is the generalized inverse. Here, $\boldsymbol{\Sigma}_{22}^{(k-1)}$ and $\boldsymbol{\Sigma}_{12}^{(k-1)}$ take the forms

$$
\boldsymbol{\Sigma}_{22}^{(k-1)}=\left[\begin{array}{cccc}
0 & 0 & 0 & \cdots \cdots \\
0 & 2^{2 H} & \left(2^{2 H}+4^{2 H}-2^{2 H}\right) / 2 & \cdots \cdots \\
0 & \left(2^{2 H}+4^{2 H}-2^{2 H}\right) / 2 & 4^{2 H} & \cdots \cdots \\
\cdots & \cdots & \cdots & \cdots \cdots \\
\cdots & \cdots & \cdots & \cdots \cdots
\end{array}\right]_{\left(2^{k-1}+1\right) \times\left(2^{k-1}+1\right)}
$$

which is a $\left(2^{k-1}+1\right)$ by $\left(2^{k-1}+1\right)$ matrix with $(i, j)$-th entry $\left(|2 i-2|^{2 H}+|2 j-2|^{2 H}-|2 i-2 j|^{2 H}\right) / 2$;

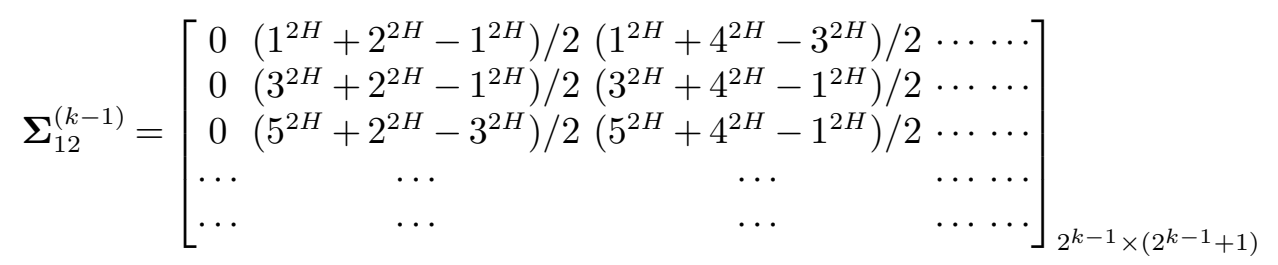

which is a $2^{k-1}$ by $\left(2^{k-1}+1\right)$ matrix with $(i, j)$-th entry $\left(|2 i-1|^{2 H}+|2 j-2|^{2 H}-|2 i-2 j+1|^{2 H}\right) / 2$. We remark that we need to use the generalized inverse in (13), because $\boldsymbol{B}_{k-1}^{H}$ is degenerate, i.e., its covariance matrix $\boldsymbol{\Sigma}_{22}^{(k-1)}$ is not full rank as $B^{H}(0)=0$. However, utilizing the generalized inverse, the conditional mean here is still well-defined (see Page 522 of Rao (1973)).

Then we have $\boldsymbol{c}_{k}-\boldsymbol{b}_{k}=\left(\boldsymbol{N}_{k-1}-\boldsymbol{M}_{k-1}\right) \boldsymbol{B}_{k-1}^{H}$ and its covariance matrix is given by

$$
\boldsymbol{\Sigma}^{(k)}=\left(\boldsymbol{N}_{k-1}-\boldsymbol{M}_{k-1}\right) \boldsymbol{\Sigma}_{22}^{(k-1)}\left(\boldsymbol{N}_{k-1}-\boldsymbol{M}_{k-1}\right)^{\top} \cdot \Delta_{k}^{2 H} .
$$

The following lemma bounds the diagonal entries of $\boldsymbol{\Sigma}^{(k)}$, which correspond to the variances of random variables $c_{j}^{k}-b_{j}^{k}, j=0,1, \cdots, 2^{k-1}-1$. 
Lemma 3. The diagonal entries of $\boldsymbol{\Sigma}^{(k)}$ are uniformly upper bounded by $2 \cdot 2^{-2 k H}$.

Then by Borell-TIS inequality, we have

$$
\mathbb{P}\left(\max _{0 \leq j \leq 2^{k-1}-1}\left|c_{j}^{k}-b_{j}^{k}\right|-\mathbb{E}\left[\max _{0 \leq j \leq 2^{k-1}-1}\left|c_{j}^{k}-b_{j}^{k}\right|\right]>\nu^{*} \ell_{k}\right) \leq \exp \left\{-\left(\nu^{*}\right)^{2} \cdot 2^{2 k \delta-2}\right\} .
$$

Similar to the proof for $(\mathrm{V})$, we can get rid of the expectation and obtain that for all $k>K(\nu)$,

$$
\mathbb{P}\left(\max _{0 \leq j \leq 2^{k-1}-1}\left|c_{j}^{k}-b_{j}^{k}\right|>\left(\nu+\nu^{*}\right) \ell_{k}\right) \leq \exp \left\{-\left(\nu^{*}\right)^{2} \cdot 2^{2 k \delta-2}\right\} .
$$

Finally, combining (12) (for (V)) and (14) (for (B)), we obtain

$$
\mathbb{P}\left(\max _{0 \leq j \leq 2^{k-1}-1}\left|a_{j}^{k}-b_{j}^{k}\right| \geq 2\left(\nu+\nu^{*}\right) \ell_{k}\right) \leq 2 \exp \left\{-\left(\nu^{*}\right)^{2} \cdot 2^{2 k \delta-2}\right\}
$$

which concludes the proof of Theorem 1.

3.1. Validity of the expansion Based on Theorem 1, we can show that the midpoint displacement approximation converges to the fBM almost surely. In particular, the next theorem establishes the validity of $(3)$.

THEOREM 2. The sample paths of $B_{n}^{H}(t)$ converge to a $f B M B^{H}(t)$ in $\mathcal{C}([0,1])$ almost surely. In other words,

$$
\mathbb{P}\left(\lim _{n \rightarrow \infty}\left\|B_{n}^{H}-B^{H}\right\|_{\infty}=0\right)=1
$$

Theorem 2 indicates that the representation

$$
B^{H}(t)-B_{n}^{H}(t)=\sum_{k=n+1}^{\infty}\left[B_{k}^{H}(t)-B_{k-1}^{H}(t)\right]
$$

is well defined. We defer the proof of Theorem 2 and proofs of other results in this section to Appendix A.

3.2. Convergence analysis in uniform norm and $\alpha$-Hölder norm In this section, we study the convergence rate of (3). We first investigate the rate of convergence in uniform norm, which provides the basis for the $\epsilon$-strong simulation of fBM. Then, for fBM with Hurst index $H>1 / 2$, we strengthen the convergence results to $\alpha$-Hölder norm, which facilitates the development of $\epsilon$-strong simulation for fBM driven SDEs.

Based on our analysis in Section 3.1, we say that a record is broken at level $k$ if

$$
\max _{0 \leq j \leq 2^{k-1}-1}\left|a_{j}^{k}-b_{j}^{k}\right| \geq \rho \ell_{k}=\rho 2^{-(H-\delta) k},
$$

and the last record breaking time $N=\sup \left\{k \geq 1: \max _{0 \leq j \leq 2^{k-1}-1}\left|a_{j}^{k}-b_{j}^{k}\right| \geq \rho \ell_{k}\right\}$. The following theorem shows that conditions (C1) and C2) are satisfied for our definition of the record-breakers.

Theorem 3. For any fixed $\delta \in(0, H)$ and $\eta>0, \mathbb{E}[\exp \{\eta N\}]<\infty$. When $n>N$,

$$
\left\|B^{H}-B_{n}^{H}\right\|_{\infty} \leq \frac{\rho \cdot 2^{-(H-\delta)(n+1)}}{\left(1-2^{-(H-\delta)}\right)} .
$$


Note that Theorem 3 implies that the level of the last record-breaker $N$ is finite almost surely.

For the $\alpha$-Hölder norm, we only consider fBM with Hurst index $H>1 / 2$. In this case, for any $\alpha \in(1 / 2, H)$, the sample paths of $B^{H}$ are $\alpha$-Hölder continuous almost surely. By the representation (15), we have the following upper bound for the $\alpha$-Hölder norm of the discretization error

$$
\left\|B_{n}^{H}-B^{H}\right\|_{\alpha} \leq \sum_{k=n+1}^{\infty}\left\|B_{k}^{H}-B_{k-1}^{H}\right\|_{\alpha} .
$$

For each discretization level $k$, the following lemma provides a computable bound for $\left\|B_{k}^{H}-B_{k-1}^{H}\right\|_{\alpha}$.

Lemma 4. For all $k \geq 1$, we have

$$
\left\|B_{k}^{H}-B_{k-1}^{H}\right\|_{\alpha} \leq 2^{\alpha(k-1)+2} \cdot \max _{0 \leq j \leq 2^{k-1}-1}\left|a_{j}^{k}-b_{j}^{k}\right| .
$$

The following theorem establishes convergence rate of (3) in the $\alpha$-Hölder norm.

Theorem 4. Consider $H>1 / 2$. For any fixed $\alpha \in(1 / 2, H)$ and $\delta \in(0, H-\alpha)$, when $n>N$

$$
\left\|B^{H}-B_{n}^{H}\right\|_{\alpha} \leq \frac{\rho 2^{2-\alpha} \cdot 2^{-(H-\alpha-\delta)(n+1)}}{1-2^{-(H-\alpha-\delta)}} .
$$

As a result of Theorem 4, once we find $N$, we also have an upper bound for the $\alpha$-Hölder norm of the fBM sample path. Specifically,

$$
\left\|B^{H}\right\|_{\alpha} \leq\left\|B_{N}^{H}\right\|_{\alpha}+\frac{\rho 2^{2-\alpha} \cdot 2^{-(H-\alpha-\delta)(N+1)}}{1-2^{-(H-\alpha-\delta)}} .
$$

4. Simulation Algorithm In this section, we introduce our $\epsilon$-strong simulation algorithm in details. As discussed in Section 2, our simulation algorithm includes two main steps. First, we simulate the fBM up to level $N$, where $N$ is the level of the last record-breaker. Notice that once we find $N$, the truncation error at level $n>N$, is controlled by

$$
\rho \cdot \sum_{k=n+1}^{\infty} 2^{-(H-\delta) k}=\frac{\rho \cdot 2^{-(H-\delta)(n+1)}}{1-2^{-(H-\delta)}} .
$$

Second, we find the truncation level $N(\epsilon)$ such that $\left(1-2^{-(H-\delta)}\right)^{-1} \cdot \rho \cdot 2^{-(H-\delta)(N(\epsilon)+1)} \leq \epsilon$. In this step, if $N(\epsilon) \leq N$, we have already obtained an $\epsilon$-strong approximated sample path of fBM by simulating the path up to level $N$. Otherwise, we need to refine the path from level $N$ to level $N(\epsilon)$. We summarize our main simulation algorithm in Algorithm 1.

The details of the first step (finding the last record-breaker) is further outlined in Algorithm 2. The second step (refining the dyadic approximation up to the desired truncation level) involves simple acceptance-rejection method, and is already detailed in Algorithm 1. We note that sampling the fBM at $D_{N(\epsilon)}$ given its values at $D_{N}$ can be implemented straightforwardly by Cholesky decomposition given the conditional mean and covariance matrix. However, this implementation has a high computational cost. We will provide a more efficient recursive construction in Section 4.3.

In Algorithm 2, we find the record-breakers sequentially until the last one. Finding the next record-breaker is a challenging task, as the next record-breaker may never happen. We overcome the difficulty here by using techniques from rare-event simulation. Intuitively, breaking the record at level $n$ is a rare event for large values of $n$. Thus, we need to find a proper change-of-measure to induce such an event. Then we can use acceptance-rejection method to decide whether to accept the record-breaking path or to reject the path and claim that the record-breaker will never happen again. More details of how to find the next record-breaker are summarized in Algorithm 3. 


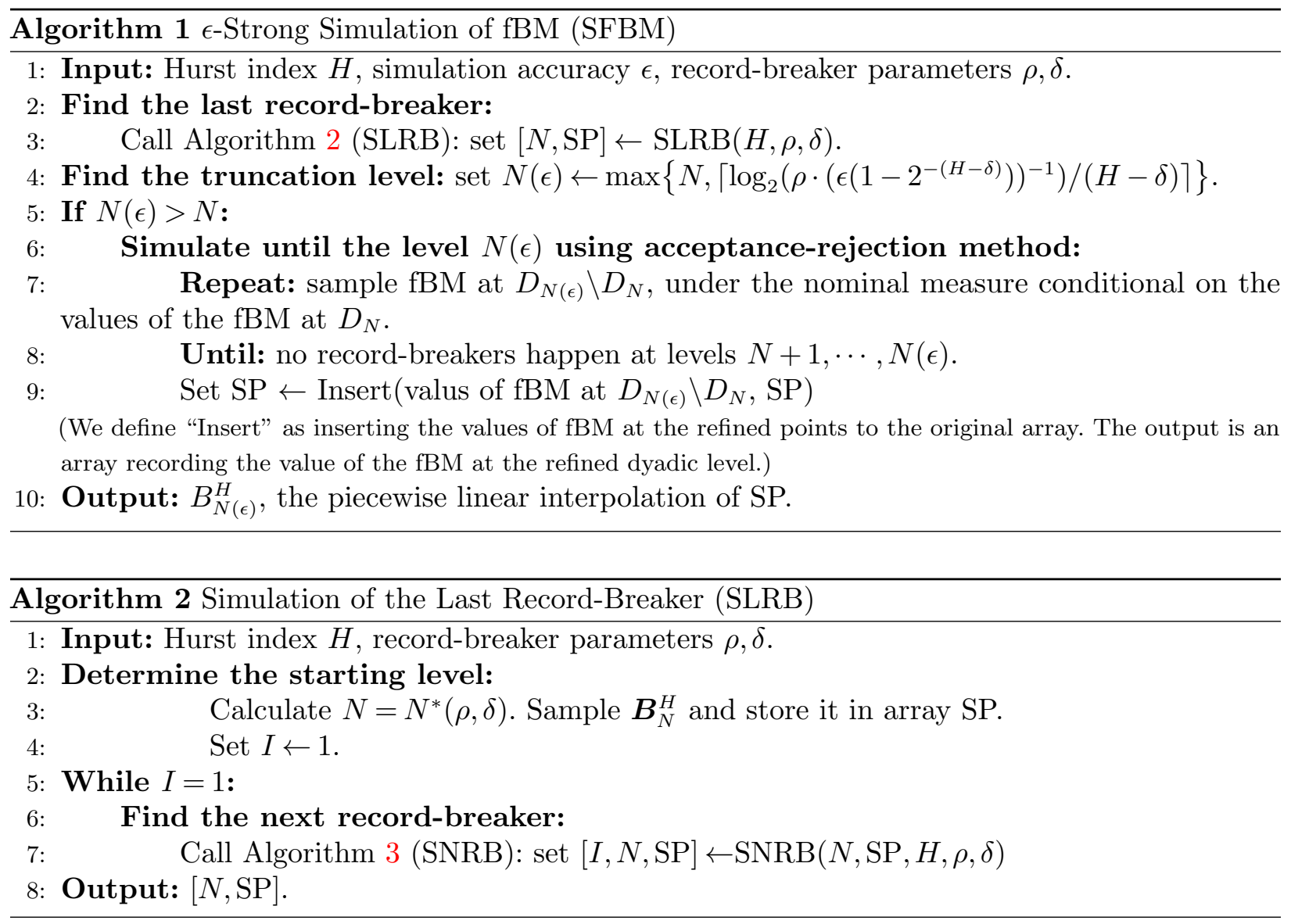

REMARK 1. In Algorithm 2, we need to start the dyadic approximation from a nontrivial starting level $N^{*}(\rho, \delta)$, which is defined later in equation (25) for technical reasons. Specifically, $N^{*}(\rho, \delta)$ is introduced to ensure that the likelihood ratio induced by the change-of-measure in Algorithm 3 can be properly bounded. Note that $N^{*}(\rho, \delta)$ depends on our choice of $(\rho, \delta)$. We provide more discussions about it in Section 6.

In Algorithm 3, we apply a change-of-measure technique to find the next record-breaker or claim that the record will never be broken again. The basic idea is as follows. Assuming that we have already simulated the fBM up to level $n$. We denote by $\tau>n$ the level of the next record-breaker. Our goal is to find the next record-breaker or claim that $\tau=\infty$, which means that the record will never be broken again. In order to determine whether $\tau<\infty$, we essentially want to generate a Bernoulli random variable with success probability $\mathbb{P}_{n}(\tau<\infty):=\mathbb{P}\left(\tau<\infty \mid \boldsymbol{B}_{n}^{H}\right)$. However, the exact value of $\mathbb{P}_{n}(\tau<\infty)$ is intractable. To overcome this difficulty, note that we can rewrite the probability as

$$
\mathbb{P}_{n}(\tau<\infty)=\sum_{m=1}^{\infty} \mathbb{P}_{n}(\tau=n+m)=\sum_{m=1}^{\infty} \frac{\mathbb{P}_{n}(\tau=n+m)}{g_{n}(m)} \cdot g_{n}(m),
$$

where $\left\{g_{n}(m)\right\}_{m \geq 1}$ is a carefully designed distribution taking values in $\mathbb{Z}^{+}$such that

$$
\mathbb{P}_{n}(\tau=n+m) / g_{n}(m) \leq 1,
$$

for all $m \geq 1$. $\left\{g_{n}(m)\right\}_{m \geq 1}$ can be interpreted as a potential realization of $\tau-n$.

We now introduce our specific choice of $\left\{g_{n}(m)\right\}_{m \geq 1}$.

$$
g_{n}(m)=Z_{n}^{-1} \cdot 2^{n+m} \cdot \exp \left\{-\rho^{2} / 8 \cdot 2^{2(n+m) \delta}\right\},
$$


where $Z_{n}$ is the normalizing constant. If we first generate $M$ from $\left\{g_{n}(m)\right\}_{m \geq 1}$ and then, given $M=m$, generate a Bernoulli random variable with success probability $\mathbb{P}_{n}(\tau=n+m) / g_{n}(m)$, then, it is easy to see from equation (17) that we obtain a Bernoulli random variable with success probability $\mathbb{P}_{n}(\tau<\infty)$. Moreover, if the Bernoulli trial is a success, we also know that the next record-breaker will happen at level $m$.

We next explain the strategy as how to generate a Bernoulli random variable with success probability $\mathbb{P}_{n}(\tau=n+m) / g_{n}(m)$. We first note that as $\mathbb{P}_{n}(\tau=n+m)$ is unknown, we can not evaluate the success probability explicitly. However, by generating the fBM up to level $n+m$, we can check whether $\tau=n+m$. Thus, we can draw Bernoulli random variables with probability of success $\mathbb{P}_{n}(\tau=n+m)$. As we know $g_{n}(m)$ explicitly, if $g_{n}(m)$ is properly chosen, we can potentially apply the Bernoulli factory to sample Bernoulli random variables with success probability $\mathbb{P}_{n}(\tau=n+m) / g_{n}(m)$ (Huber, 2016). In our algorithmic development, we take a simpler route than the Bernoulli factory by applying the change-of-measure technique. The key idea is to construct a new measure $\mathbb{Q}_{n}^{(m)}$ such that

$$
\frac{\mathrm{d} \mathbb{P}_{n}}{\mathrm{~d} \mathbb{Q}_{n}^{(m)}} 1\{\tau=n+m\} \leq g_{n}(m) .
$$

In particular, we first sample the fBM up to level $m$, from $\mathbb{Q}_{n}^{(m)}$. Then, as

$$
\mathbb{E}_{\mathbb{Q}_{n}^{(m)}}\left[\frac{\mathrm{d} \mathbb{P}_{n}}{\mathrm{~d} \mathbb{Q}_{n}^{(m)}} \cdot \frac{1\{\tau=n+m\}}{g_{n}(m)}\right]=\frac{\mathbb{P}_{n}(\tau=n+m)}{g_{n}(m)}
$$

we can generate $U$, a uniform random variable over $[0,1]$ independent of everything else, and check whether

$$
U<\frac{\mathrm{d} \mathbb{P}_{n}}{\mathrm{~d} \mathbb{Q}_{n}^{(m)}} \cdot \frac{1\{\tau=n+m\}}{g_{n}(m)} .
$$

In particular, it is easy to check that when the inequality (20) is satisfied, (21) holds with probability $\mathbb{P}_{n}(\tau=n+m) / g_{n}(m)$. Moreover, if the inequality (21) holds, we accept the proposed trajectory as one that leads to the next record-breaker, i.e., we get $\boldsymbol{B}_{\tau}^{H}$; Otherwise, we claim that $\tau=\infty$. Lastly, we comment that for the bound (20) to hold, we need to make sure that when $\tau=n+m$, $\mathrm{dP}_{n}$ is much smaller than $\mathrm{d} \mathbb{Q}_{n}^{(m)}$, since by $(19), g_{n}(m)$ is very small for large values of $n$ and $m$. This implies that the new measure $\mathbb{Q}_{n}^{(m)}$ needs to be properly constructed such that the record-breaking event is more likely to happen under $\mathbb{Q}_{n}^{(m)}$. As record-breaking is rare under $\mathbb{P}_{n}$, we apply exponential tiling (a rare event simulation technique) to construct $\mathbb{Q}_{n}^{(m)}$.

The actual construction of the change-of-measure involves more subtleties. For example, we not only need to consider the level of the next record-breaker, $n+m$, but also the actual time index, and whether we break the record due to a large positive deviation or negative deviation. The details of these subtleties are deferred to Section 4.1. It is also in general not easy to bound the probability ratio (18) and likelihood ratio $\mathrm{dP}_{n} / \mathrm{d} \mathbb{Q}_{n}^{(m)}$, even with carefully chosen $g_{n}(m)$ and $\mathbb{Q}_{n}^{(m)}$. We thus introduce another technical step in the algorithm - the "bounded conditional expectation" condition (BCE). Let

$$
\boldsymbol{\beta}=(1 / 2,-1,1 / 2)^{\top}, \boldsymbol{\alpha}_{n}(m, k)=\left(B^{H}\left(t_{2 k-2}^{n+m}\right), B^{H}\left(t_{2 k-1}^{n+m}\right), B^{H}\left(t_{2 k}^{n+m}\right)\right)^{\top} .
$$

and

$$
\bar{\mu}_{n}(m, k)=\boldsymbol{\beta}^{\top} \mathbb{E}\left[\boldsymbol{\alpha}_{n}(m, k) \mid \boldsymbol{B}_{n}^{H}\right] .
$$


Definition 1. (BCE condition) We say that $\boldsymbol{B}_{n}^{H}$ satisfies the bounded conditional expectation condition, if for all $m \geq 1$ and $1 \leq k \leq 2^{n+m-1}$,

$$
\left|\bar{\mu}_{n}(m, k)\right|=\left|\boldsymbol{\beta}^{\top} \mathbb{E}\left[\boldsymbol{\alpha}_{n}(m, k) \mid \boldsymbol{B}_{n}^{H}\right]\right| \leq \rho / 2 \cdot \ell_{n+m},
$$

We will show in Section 4.1 that if the BCE condition is satisfied at level $n$, then the probability ratio (18) is upper bounded by one, given that $n$ is large enough such that $Z_{n} \leq 1$. Moreover, the likelihood ratio in (20) is also properly bounded if we apply the change-of-measure from level $n+1$ onwards. In addition, this condition only gets violated a finite number of times almost surely. When simulating the next record-breaker, we will first check if $\boldsymbol{B}_{n}^{H}$ satisfies the BCE condition. If not, we will keep generating more refined levels under the nominal measure until the BCE condition is satisfied. Only then, we apply the change-of-measure to find the next record-breaker. The details of how to check whether the BCE condition is met are laid out in Algorithm 5, which we defer to Section 4.1 after we introduce a few more technical results.

REMARK 2. We comment that the idea of introducing an intermediate condition, e.g., the $\mathrm{BCE}$ condition in our case, can be of interests to the development of other rare-event simulation algorithms. Note that the key in successful applications of the change-of-measure technique is that the likelihood ratio function is suitably bounded. The intermediate condition we introduce satisfies two important properties: 1) if this condition is satisfied, then the likelihood ratio is properly bounded; 2) this condition is only violated a finite number of times. Similar intermediate conditions that satisfy the two important properties can be used elsewhere.

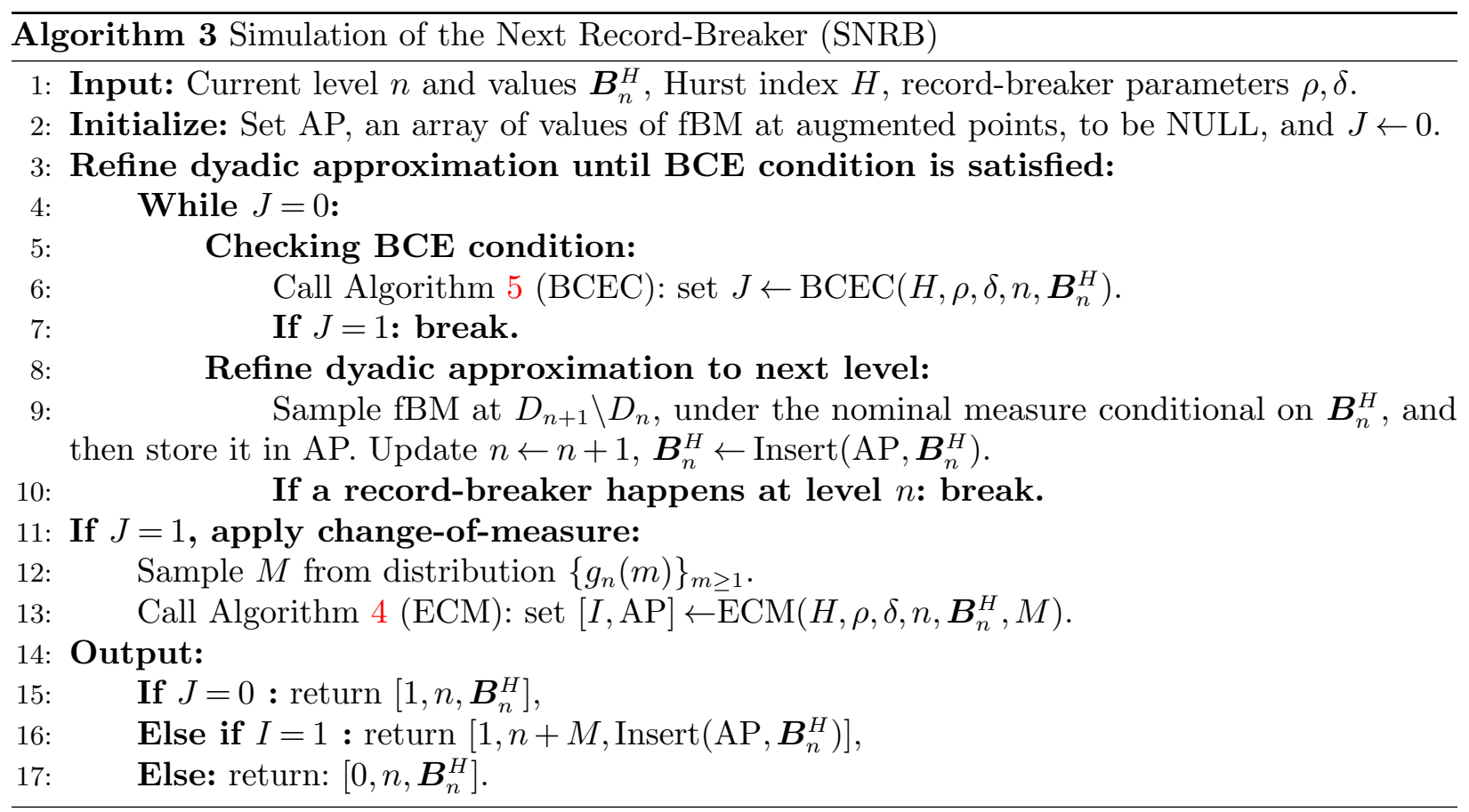

4.1. Change of measure In this section, we provide details of our construction of the new measure under which the record-breaker is more likely to happen. Recall that the setting is that we have already generated $\boldsymbol{B}_{n}^{H}$ and a proposed next record breaking level $n+m$, where $m$ is sampled from the distribution $\left\{g_{n}(m)\right\}_{m \in \mathbb{Z}^{+}}$. Our goal is to find a way to generate a path such that the next record-breaker is more likely to happen at level $n+m$. This will ensure that the likelihood ratio, $\mathrm{d} \mathbb{P}_{n} / \mathbb{Q}_{n}^{(m)}$, can be properly bounded. 
Based on the definition of $\boldsymbol{\alpha}_{n}(m, k)$ and $\boldsymbol{\beta}$ in equation (22), we say that a record is broken at level $n+m$, position $k$, if

$$
\left|\boldsymbol{\beta}^{\top} \boldsymbol{\alpha}_{n}(m, k)\right|>\rho \ell_{n+m}=\rho 2^{-(H-\delta)(n+m)} .
$$

Furthermore, we say that the record-breaker is up-crossing if

$$
\boldsymbol{\beta}^{\top} \boldsymbol{\alpha}_{n}(m, k)>\rho \ell_{n+m},
$$

and downward-crossing if

$$
\boldsymbol{\beta}^{\top} \boldsymbol{\alpha}_{n}(m, k)<-\rho \ell_{n+m}
$$

Let

$$
\Xi_{n}^{(m, k)}(\theta)=\mathbb{E}_{n}\left[\exp \left\{\theta \cdot \boldsymbol{\beta}^{\top} \boldsymbol{\alpha}_{n}(m, k)\right\}\right]=\mathbb{E}\left[\exp \left\{\theta \cdot \boldsymbol{\beta}^{\top} \boldsymbol{\alpha}_{n}(m, k)\right\} \mid \boldsymbol{B}_{n}^{H}\right]
$$

which is the moment generating function of $\boldsymbol{\beta}^{\top} \boldsymbol{\alpha}_{n}(m, k)$ conditional on the value of $\boldsymbol{B}_{n}^{H}$. We denote the conditional probability density of $\boldsymbol{\alpha}_{n}(m, k)$ under measure $\mathbb{P}_{n}(\cdot)$ by $\psi_{n}^{(m, k)}$. We also denote

$$
\boldsymbol{\mu}_{n}(m, k)=\mathbb{E}_{n}\left[\boldsymbol{\alpha}_{n}(m, k)\right] \text { and } \boldsymbol{\Sigma}_{\boldsymbol{\alpha}_{n}(m, k)}=\mathbb{E}_{n}\left[\left(\boldsymbol{\alpha}_{n}(m, k)-\boldsymbol{\mu}_{n}(m, k)\right)\left(\boldsymbol{\alpha}_{n}(m, k)-\boldsymbol{\mu}_{n}(m, k)\right)^{\top}\right]
$$

i.e., the conditional expectation and covariance of $\boldsymbol{\alpha}_{n}(m, k)$ respectively. Note that $\bar{\mu}_{n}(m, k)=$ $\beta^{\top} \boldsymbol{\mu}_{n}(m, k)$.

In what follows, we start by introducing the change-of-measure under the BCE condition. We then show that the BCE condition can only be violated a finite number of times almost surely. Under the BCE condition, we first sample $K$ from the set $\left\{1,2, \cdots, 2^{n+m-1}\right\}$ uniformly. The random variable $K$ proposes the position of the next record-breaker. We also sample $\Pi$ from the set $\{+1,-1\}$ uniformly. The random variable $\Pi$ proposes whether the record-breaker is up-crossing $(+1)$ or downward-crossing (-1). Second, given $M=m, K=k$, and $\Pi=\pi$, we apply exponential tilting to $\psi_{n}^{(m, k)}$ with tilting parameter

$$
\theta_{n}^{\pi}(m)=\pi \rho / 2 \cdot 2^{(m+n)(H+\delta)} .
$$

We remark that the tilting parameter $\theta_{n}^{\pi}(m)$ is chosen to make sure that the record-breaking event is more likely to happen under the tilted measure and the likelihood ratio is properly bounded, i.e., the bound (20) holds (see Lemma 5 for more details).

Under the exponential tilting, we sample $\boldsymbol{\alpha}_{n}(m, k)$ from the density

$$
\tilde{\psi}_{n}^{(m, k, \pi)}\left(x_{1}, x_{2}, x_{3}\right)=\psi_{n}^{(m, k)}\left(x_{1}, x_{2}, x_{3}\right) \cdot \exp \left\{\theta_{n}^{\pi}(m) \cdot\left(\frac{1}{2}\left(x_{1}+x_{3}\right)-x_{2}\right)-\log \left(\Xi_{n}^{(m, k)}\left(\theta_{n}^{\pi}(m)\right)\right)\right\} .
$$

Note that the tilted distribution $\tilde{\psi}_{n}^{(m, k, \pi)}$ is still Gaussian. In particular, $\tilde{\psi}_{n}^{(m, k, \pi)}$ is the density of the multivariate Gaussian with mean $\boldsymbol{\mu}_{n}(m, k)+\theta_{n}^{\pi}(m) \cdot \boldsymbol{\Sigma}_{\boldsymbol{\alpha}_{n}(m, k)} \boldsymbol{\beta}$ and variance $\boldsymbol{\Sigma}_{\boldsymbol{\alpha}_{n}(m, k)}$.

After we have sampled $\boldsymbol{\alpha}_{n}(m, k)$ under the tilted measure, given the values of $\boldsymbol{\alpha}_{n}(m, k)$ and $\boldsymbol{B}_{n}^{H}$, we sample $\mathrm{fBM}$ at the remained dyadic points

$$
D_{n+m} \backslash\left(D_{n} \cup\left\{t_{2 k-2}^{n+m}, t_{2 k-1}^{n+m}, t_{2 k}^{n+m}\right\}\right)
$$

under the nominal measure. This step is achieved by calculating the conditional expectation and covariance matrix, and then sampling from the corresponding multivariate Gaussian distribution.

We use $\mathbb{Q}_{n}$ to denote the new measure induced by the above sampling scheme. Specifically, $\mathbb{Q}_{n}$ involves sampling $M, K$, and $\Pi$, using exponential tilting to sample $\boldsymbol{\alpha}_{n}(m, k)$, and sampling the fBM at $D_{n+m} \backslash\left(D_{n} \cup\left\{t_{2 k-2}^{n+m}, t_{2 k-1}^{n+m}, t_{2 k}^{n+m}\right\}\right)$ conditional on $\boldsymbol{B}_{n}^{H}$ and $\boldsymbol{\alpha}_{n}(m, k)$. We also denote $\mathbb{Q}_{n}^{(m, k, \pi)}$ as the conditional probability measure $\mathbb{Q}_{n}(\cdot \mid M=m, K=k, \Pi=\pi)$. Recall that we sample $M$ from 
the distribution $\left\{g_{n}(m)\right\}_{m \geq 1}$ and $K, \Pi$ uniformly at random. Thus $\mathrm{d} \mathbb{Q}_{n}^{(m, k, \pi)}=\mathrm{d} \mathbb{Q}_{n} \cdot 1\{M=m, K=$ $k, \Pi=\pi\} /\left(g_{n}(m) \cdot 2^{-(n+m)}\right)$. Then the likelihood ratio between the measures $\mathbb{P}_{n}$ and $\mathbb{Q}_{n}$ on the event $M=m, K=k$ and $\Pi=\pi$ is

$$
\begin{aligned}
\Theta_{n}^{\pi}(m, k) & :=\mathrm{d} \mathbb{P}_{n} / \mathrm{d} \mathbb{Q}_{n} \cdot 1\{M=m, K=k, \Pi=\pi\} \\
& =\mathrm{d} \mathbb{P}_{n} / \mathrm{d} \mathbb{Q}_{n}^{(m, k, \pi)} \cdot g_{n}(m)^{-1} \cdot 2^{n+m} \\
& =g_{n}(m)^{-1} \cdot 2^{n+m} \cdot \exp \left\{-\theta_{n}^{\pi}(m) \cdot \boldsymbol{\beta}^{\top} \boldsymbol{\alpha}_{n}(m, k)+\log \left(\Xi_{n}^{(m, k)}\left(\theta_{n}^{\pi}(m)\right)\right)\right\} .
\end{aligned}
$$

From the definition of $g_{n}(m)$ in (19), the normalizing constant $Z_{n}$ is given by

$$
Z_{n}=\sum_{m=1}^{\infty} 2^{n+m} \cdot \exp \left\{-\rho^{2} / 8 \cdot 2^{2(n+m) \delta}\right\} .
$$

We define

$$
N^{*}(\rho, \delta)=1+\sup \left\{n \geq 1: Z_{n}>1\right\} .
$$

Note that for all $n \geq N^{*}(\rho, \delta)$, we have $Z_{n} \leq 1$. The next lemma shows that under the BCE condition, $\Theta_{n}^{\pi}(m, k)$ is suitably bounded. This result is important in constructing our change-of-measure procedure.

Lemma 5. For $n \geq N^{*}(\rho, \delta)$, under the $B C E$ condition, when $M=m, K=k$, and $\Pi=\pi$,

$$
\Theta_{n}^{\pi}(m, k) \cdot 1\left\{\pi \cdot \beta^{\top} \boldsymbol{\alpha}_{n}(m, k)>\rho \ell_{n+m}\right\} \leq 1 .
$$

The proof of Lemma 5 and subsequent lemmas in this section can be found in Appendix B.

We are now ready to present our actual algorithm. Let $\mathcal{C}_{n}(j)$ denotes the event that there are record-breakers at level $n+j$. We also write

$$
R_{n+m}=\sum_{k=1}^{2^{n+m-1}} 1\left\{\left|\boldsymbol{\beta}^{\top} \boldsymbol{\alpha}_{n}(m, k)\right|>\rho \ell_{n+m}\right\},
$$

as the total number of record-breakers at level $n+m$. We generate a uniformly distributed random variable $U$ over interval $[0,1]$, independent of everything else. Then, we return 1 if

$$
U<\Theta_{n}^{\pi}(m, k) \cdot R_{n+m}^{-1} \cdot 1\left\{\left\{\pi \cdot \boldsymbol{\beta}^{\top} \boldsymbol{\alpha}_{n}(m, k)>\rho \ell_{n+m}\right\} \cap \cap_{j=1}^{m-1}\left[\mathcal{C}_{n}(j)\right]^{c}\right\},
$$

and return 0 otherwise. We define $0 / 0=0$ by convention. The details of this simulation procedure are summarized in Algorithm 4.

It remains to show that the $\mathrm{BCE}$ condition is only violated a finite number of times and construct an efficient way to check whether the BCE condition is satisfied at a specific level $n$. Let

$$
\mathcal{E}_{n}=\left\{\left|\bar{\mu}_{n}(m, k)\right|>\rho / 2 \cdot \ell_{n+m}, \text { for some } m \geq 1 \text { and } 1 \leq k \leq 2^{n+m-1}\right\},
$$

where $\bar{\mu}_{n}(m, k)$ is the conditional expectation defined in (23). By Definition $1, \mathcal{E}_{n}$ denotes the event that the $\mathrm{BCE}$ condition is violated at level $n$. We also define

$$
\mathcal{N}_{\mathcal{E}}=\sup \left\{n \geq 1: \mathcal{E}_{n} \text { happens }\right\},
$$

i.e., it is the last level at which the $\mathrm{BCE}$ condition is violated.

Lemma 6. For any fixed $\delta \in(0, H)$ and $\eta>0, \mathbb{E}\left[\exp \left\{\eta \mathcal{N}_{\mathcal{E}}\right\}\right]<\infty$. 


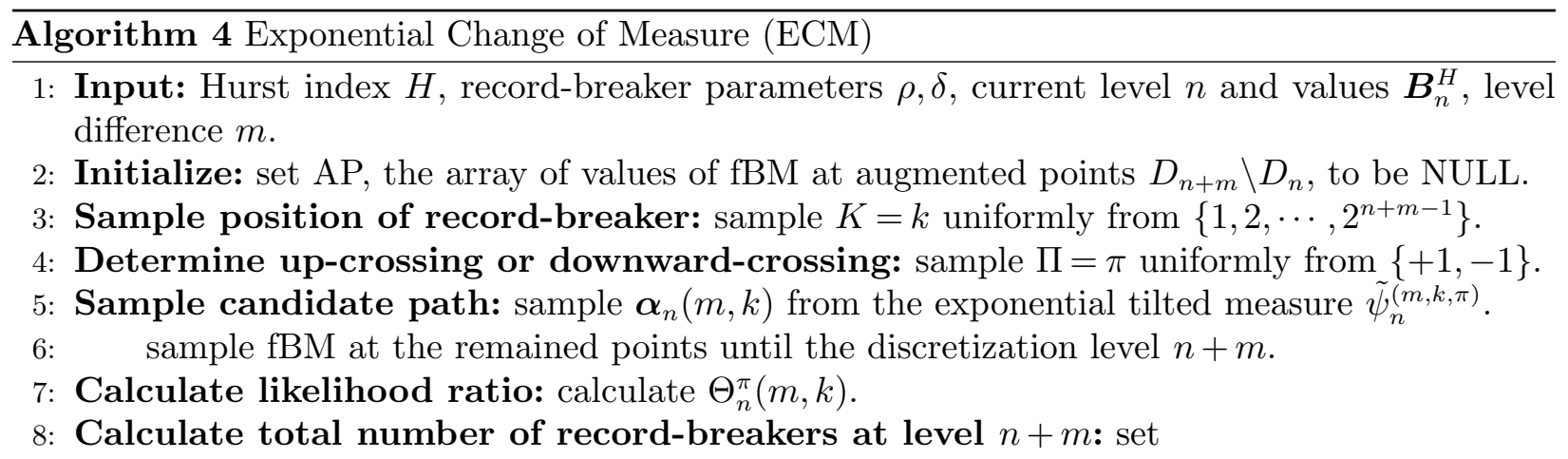

$$
R_{n+m} \leftarrow \sum_{k=1}^{2^{n+m-1}} 1\left\{\left|\boldsymbol{\beta}^{\top} \boldsymbol{\alpha}_{n}(m, k)\right|>\rho \ell_{n+m}\right\} .
$$

9: Sample from uniform distribution: generate $U \sim \mathcal{U}[0,1]$.

10: Determine the next record-breaker via acceptance-rejection: set

$$
I \leftarrow 1\left\{U<\Theta_{n}^{\pi}(m, k) \cdot R_{n+m}^{-1} \cdot 1\left\{\left\{\pi \cdot \boldsymbol{\beta}^{\top} \boldsymbol{\alpha}_{n}(m, k)>\rho \ell_{n+m}\right\} \cap\left\{\cap_{j=1}^{m-1}\left[\mathcal{C}_{n}(j)\right]^{c}\right\}\right\}\right\} .
$$

11: If $I=1: \mathrm{AP}=$ values of $\mathrm{fBM}$ at augmented points $D_{n+m} \backslash D_{n}$.

12: Output: $I$ and AP.

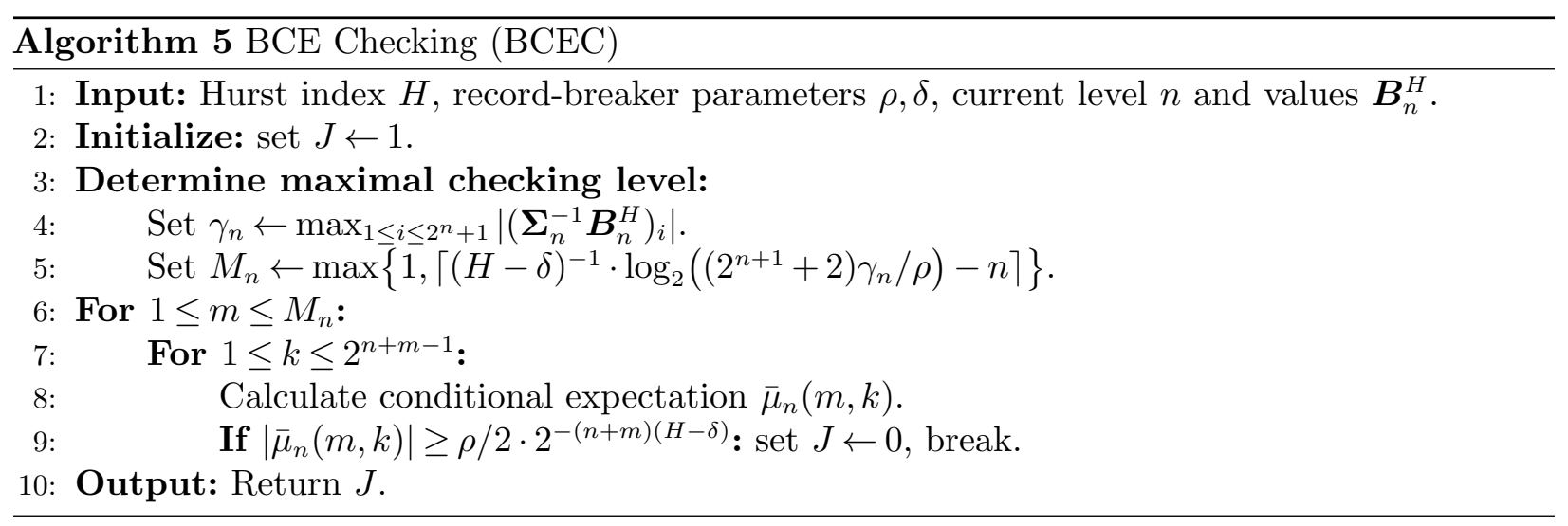

Lemma 6 implies that $\mathbb{P}\left(\mathcal{N}_{\mathcal{E}}<\infty\right)=1$, i.e., The BCE condition is violated a finite number times almost surely. We also remark that like $N$, the time of the last record-breaker, $\mathcal{N}_{\mathcal{E}}$ is not a stopping time. However, in our algorithm, we do not need to find $\mathcal{N}_{\mathcal{E}}$. We only need to check whether the BCE condition is violated at a particular level $n$.

We next introduce the algorithm to check whether the BCE condition is satisfied at level $n$ given the value of $\boldsymbol{B}_{n}^{H}$. The development of the algorithm is highly nontrivial. In particular, directly checking by definition is impractical, as we needs to evaluate $\bar{\mu}_{n}(m, k)$ for $m=1,2, \ldots$, $k=1,2, \ldots, 2^{n+m-1}$, i.e., infinitely many terms. To overcome this difficulty, we need to further explore the structure of $\bar{\mu}_{n}(m, k)$. We use $\gamma_{n}$ to denote the maximal absolute value of the entries in vector $\boldsymbol{\Sigma}_{n}^{-1} \boldsymbol{B}_{n}^{H}$, where $\boldsymbol{\Sigma}_{n}$ is the covariance matrix of $\boldsymbol{B}_{n}^{H}$. We have the following lemma which establishes a bound for $\bar{\mu}_{n}(m, k)$. 
Lemma 7. For all $m \geq 1$ and $1 \leq k \leq 2^{n+m-1}$, we have

$$
\left|\bar{\mu}_{n}(m, k)\right| \leq \gamma_{n} \cdot\left(2^{n}+1\right) \cdot 2^{-2(n+m) H} .
$$

According to Lemma 7, for a fixed level $n$ and given value of $\boldsymbol{B}_{n}^{H}$, the decay rate of $\left|\bar{\mu}_{n}(m, k)\right|$ (with respect to $m$ ) is $O\left(2^{-2 m H}\right)$. However, to check the BCE condition, we only need to compare $\left|\bar{\mu}_{n}(m, k)\right|$ with $\rho / 2 \cdot 2^{-(n+m)(H-\delta)}$, whose decay rate (with respect to $m$ ) is $O\left(2^{-m(H-\delta)}\right)$. Hence, when

$$
m \geq M_{n}=\max \left\{1,\left\lceil(H-\delta)^{-1} \cdot \log _{2}\left(\left(2^{n+1}+2\right) \gamma_{n} / \rho\right)-n\right\rceil\right\}
$$

the following inequality

$$
\left|\bar{\mu}_{n}(m, k)\right| \leq \rho / 2 \cdot 2^{-(n+m)(H-\delta)}
$$

always holds. This implies that to check whether BCE condition holds at level $n$, we only need to calculate a finite collection of $\bar{\mu}_{n}(m, k)$, i.e. $m=1, \ldots, M_{n}, k=1,2, \ldots, 2^{n+m-1}$. The details is summarized in Algorithm 5, which outputs 1 when the BCE condition is satisfied and 0, otherwise.

4.2. Correctness of the algorithm In this section, we provide detailed proof to show that our algorithm actually works. The proof is divided into two main steps (two theorems). We first show that under the BCE condition, for the output of Algorithm 3, $\mathbb{Q}_{n}(M=m, I=1)=\mathbb{P}_{n}(\tau=n+m)$ and $\mathbb{Q}_{n}(I=0)=\mathbb{P}_{n}(\tau=\infty)$ (Theorem 5). We then show that when $M=m$ and $I=1$, the output path of Algorithm 4, i.e., the fBM at the augmented points $D_{n+m} \backslash D_{n}$, follows from the distribution $\mathbb{P}_{n}(\cdot \mid \tau=n+m)$ (Theorem 6$)$.

THEOREM 5. For the output of Algorithm 3, when $n \geq N^{*}(\rho, \delta)$ and the BCE condition holds, $I$ is a Bernoulli random variable with success probability $\mathbb{P}_{n}(\tau<\infty)$. Moreover,

$$
\mathbb{Q}_{n}(M=m, I=1)=\mathbb{P}_{n}(\tau=n+m) .
$$

Proof. By definition, we have

$$
\mathbb{Q}_{n}(I=1)=\sum_{m=1}^{\infty} \mathbb{Q}_{n}(I=1, M=m)=\sum_{m=1}^{\infty} \mathbb{Q}_{n}(I=1 \mid M=m) \cdot g_{n}(m) .
$$

We next show that $\mathbb{Q}_{n}(I=1 \mid M=m)=\mathbb{P}_{n}(\tau=n+m) / g_{n}(m)$. Note that if $X$ is a random variable taking value in $[0,1]$ and $U$ is a uniformly distributed random variable on $[0,1]$ independent of $X$, then $1\{U<X\}$ is a Bernoulli random variable of success probability $\mathbb{E}[X]$. Recall that in our algorithm, both $\Theta_{n}^{\pi}(m, k)$ is bounded by one. Then by the definition of $I$ and Lemma 5 , we have the following decomposition

$$
\begin{aligned}
& \mathbb{Q}_{n}(I=1 \mid M=m) \\
& =\sum_{\pi=+,-} \mathbb{E}_{\mathbb{Q}_{n}^{(m)}}\left[1\left\{\left\{\pi \cdot \boldsymbol{\beta}^{\top} \boldsymbol{\alpha}_{n}(m, K)>\rho \ell_{n+m}\right\} \cap\left\{\cap_{j=1}^{m-1}\left[\mathcal{C}_{n}(j)\right]^{c}\right\}\right\} \cdot \Theta_{n}^{\pi}(m, K) \cdot R_{n+m}^{-1} \mid \pi\right] \cdot \frac{1}{2} \\
& =\sum_{\pi=+,-} \sum_{k=1}^{2^{n+m-1}} \mathbb{E}_{\mathbb{Q}_{n}^{(m, k, \pi)}}\left[1\left\{\left\{\pi \cdot \boldsymbol{\beta}^{\top} \boldsymbol{\alpha}_{n}(m, k)>\rho \ell_{n+m}\right\} \cap\left\{\cap_{j=1}^{m-1}\left[\mathcal{C}_{n}(j)\right]^{c}\right\}\right\} \cdot \Theta_{n}^{\pi}(m, k) \cdot R_{n+m}^{-1}\right] \cdot \frac{1}{2^{n+m}}
\end{aligned}
$$

By the definition of weighted likelihood ratio $\Theta_{n}^{\pi}(m, k)$, we further have

$$
\begin{aligned}
& \mathbb{Q}_{n}(I=1 \mid M=m) \\
& =\sum_{\pi=+,-} \sum_{k=1}^{2^{n+m-1}} \mathbb{E}_{\mathbb{Q}_{n}^{(m, k, \pi)}}\left[1\left\{\left\{\pi \cdot \boldsymbol{\beta}^{\top} \boldsymbol{\alpha}_{n}(m, k)>\rho \ell_{n+m}\right\} \cap\left\{\cap_{j=1}^{m-1}\left[\mathcal{C}_{n}(j)\right]^{c}\right\}\right\} \cdot R_{n+m}^{-1} \cdot \frac{\mathrm{d} \mathbb{P}_{n}}{\mathrm{~d} \mathbb{Q}_{n}^{(m, k, \pi)}} \cdot g_{n}(m)^{-1}\right] .
\end{aligned}
$$


Note that as

$$
1\left\{\left|\boldsymbol{\beta}^{\top} \boldsymbol{\alpha}_{n}(m, k)\right|>\rho \ell_{n+m}\right\}=1\left\{\boldsymbol{\beta}^{\top} \boldsymbol{\alpha}_{n}(m, k)>\rho \ell_{n+m}\right\}+1\left\{\boldsymbol{\beta}^{\top} \boldsymbol{\alpha}_{n}(m, k)<-\rho \ell_{n+m}\right\},
$$

we finally have

$$
\begin{aligned}
\mathbb{Q}_{n}(I=1 \mid M=m) & =\sum_{k=1}^{2^{n+m-1}} \mathbb{E}_{n}\left[1\left\{\left\{\left|\boldsymbol{\beta}^{\top} \boldsymbol{\alpha}_{n}(m, k)\right|>\rho \ell_{n+m}\right\} \cap \cap_{j=1}^{m-1}\left[\mathcal{C}_{n}(j)\right]^{c}\right\} \cdot R_{n+m}^{-1}\right] \cdot g_{n}(m)^{-1} \\
& =\mathbb{E}_{n}\left[1\left\{\mathcal{C}_{n}(m) \cap\left\{\cap_{j=1}^{m-1}\left[\mathcal{C}_{n}(j)\right]^{c}\right\}\right\}\right]=\mathbb{P}_{n}(\tau=n+m) / g_{n}(m) .
\end{aligned}
$$

Above all, for any $m \geq 1$, we have $\mathbb{Q}_{n}(I=1, M=m)=\mathbb{P}_{n}(\tau=n+m)$.

Based on Theorem 5, if we obtain an output $I=0$ from Algorithm 3, we claim that the recordbreaker will not happen again after level $n$. Otherwise, i.e. if we obtain $I=1$ and $M=m$ from Algorithm 3, we claim that the first next record-breaker happens at level $n+m$. In the later case, Algorithm 4 also outputs a path leading to the next record-breaker. We next show that the output of Algorithm 4 when $I=1$ is a realization of the fBM conditional on the event that the next record-breaker happens at level $n+m$.

TheOREM 6. For the output of Algorithm 4, given $M=m, I=1$, the distribution of the values of $f B M$ at the augmented points $D_{n+m} \backslash D_{n}$, follows $\mathbb{P}_{n}(\cdot \mid \tau=n+m)$.

Proof. For notational convenience, we denote by $\delta_{n}^{m} D=D_{n+m} \backslash D_{n}$ the augmented points. We also write $\delta_{n}^{m} B^{H}=\left(B^{H}(t): t \in \delta_{n}^{m} D\right)$, i.e., the fBM at the augmented points $\delta_{n}^{m} D$. Let $A$ be an arbitrary measurable set, we next show that

$$
\mathbb{P}_{n}\left(\delta_{n}^{m} B^{H} \in A \mid \tau=n+m\right)=\mathbb{Q}_{n}\left(\delta_{n}^{m} B^{H} \in A \mid I=1, M=m\right) .
$$

For $\mathbb{P}_{n}\left(\delta_{n}^{m} B^{H} \in A \mid \tau=n+m\right)$, by the definition of conditional probability, we have

$$
\mathbb{P}_{n}\left(\delta_{n}^{m} B^{H} \in A \mid \tau=n+m\right)=\frac{\mathbb{P}_{n}\left(\delta_{n}^{m} B^{H} \in A, \tau=n+m\right)}{\mathbb{P}_{n}(\tau=n+m)}=\frac{\mathbb{E}_{n}\left[1\left\{\delta_{n}^{m} B^{H} \in A\right\} \cdot 1\{\tau=n+m\}\right]}{\mathbb{P}_{n}(\tau=n+m)} .
$$

For $\mathbb{Q}_{n}\left(\delta_{n}^{m} B^{H} \in A \mid I=1, M=m\right)$, since $M$ is sampled from the distribution $\left\{g_{n}(m)\right\}_{m \geq 1}$, we have

$$
\mathbb{Q}_{n}\left(\delta_{n}^{m} B^{H} \in A \mid I=1, M=m\right)=\frac{\mathbb{Q}_{n}\left(\delta_{n}^{m} B^{H} \in A, I=1 \mid M=m\right) g_{n}(m)}{\mathbb{Q}_{n}(I=1, M=m)}
$$

We first note that by Theorem $5, \mathbb{P}_{n}(\tau=n+m)=\mathbb{Q}_{n}(U=1, M=m)$, the denominator in (27) is equal to the denominator in (28). We next show that the numerators are also equal.

For the numerator in $(27)$, recall that $\mathcal{C}_{n}(j)$ denotes the event that there is a record-breaker at level $n+j$. Then, we have $1\{\tau=n+m\}=\mathcal{C}_{n}(m) \cap\left(\cap_{j=1}^{m-1}\left[\mathcal{C}_{n}(j)\right]^{c}\right)$. We also have

$$
\mathcal{C}_{n}(m)=\left(\cup_{k=1}^{2^{n+m-1}} 1\left\{\left|\boldsymbol{\beta}^{\top} \boldsymbol{\alpha}_{n}(m, k)\right|>\rho \ell_{n+m}\right\}\right) .
$$

Then

$$
\begin{aligned}
& \mathbb{E}_{n}\left[1\left\{\delta_{n}^{m} B^{H} \in A\right\} \cdot 1\{\tau=n+m\}\right] \\
= & \sum_{\pi=+,-} \sum_{k=1}^{2^{n+m-1}} \mathbb{E}_{n}\left[1\left\{\delta_{n}^{m} B^{H} \in A\right\} \cdot R_{n+m}^{-1} \cdot 1\left\{\left\{\pi \cdot \boldsymbol{\beta}^{\top} \boldsymbol{\alpha}_{n}(m, k)>\rho \ell_{n+m}\right\} \cap\left\{\cap_{j=1}^{m-1}\left[\mathcal{C}_{n}(j)\right]^{c}\right\}\right\}\right]
\end{aligned}
$$


where $R_{n+m}$ is defined in (26). For each term in the summation in (29),

$$
\begin{aligned}
& \mathbb{E}_{n}\left[1\left\{\delta_{n}^{m} B^{H} \in A\right\} \cdot R_{n+m}^{-1} \cdot 1\left\{\left\{\pi \cdot \boldsymbol{\beta}^{\top} \boldsymbol{\alpha}_{n}(m, k)>\rho \ell_{n+m}\right\} \cap\left\{\cap_{j=1}^{m-1}\left[\mathcal{C}_{n}(j)\right]^{c}\right\}\right\}\right] \\
= & \mathbb{E}_{\mathbb{Q}_{n}^{(m, k, \pi)}}\left[1\left\{\delta_{n}^{m} B^{H} \in A\right\} \cdot 1\left\{\left\{\pi \cdot \boldsymbol{\beta}^{\top} \boldsymbol{\alpha}_{n}(m, k)>\rho \ell_{n+m}\right\} \cap\left\{\cap_{j=1}^{m-1}\left[\mathcal{C}_{n}(j)\right]^{c}\right\}\right\} \cdot R_{n+m}^{-1} \cdot \mathrm{d} \mathbb{P}_{n} / \mathrm{d} \mathbb{Q}_{n}^{(m, k, \pi)}\right] \\
= & \mathbb{E}_{\mathbb{Q}_{n}^{(m, k, \pi)}}\left[1\left\{\delta_{n}^{m} B^{H} \in A\right\} \cdot 1\left\{\left\{\pi \cdot \boldsymbol{\beta}^{\top} \boldsymbol{\alpha}_{n}(m, k)>\rho \ell_{n+m}\right\} \cap\left\{\cap_{j=1}^{m-1}\left[\mathcal{C}_{n}(j)\right]^{c}\right\}\right\} \cdot R_{n+m}^{-1} \cdot \Theta_{n}^{\pi}(m, k)\right] \frac{g_{n}(m)}{2^{n+m}},
\end{aligned}
$$

where the second equality follows from the definition of $\Theta_{n}^{\pi}(m, k)$.

For the numerator in (28), we have

$$
\begin{aligned}
\mathbb{Q}_{n}\left(\delta_{n}^{m} B^{H} \in A, I=1 \mid M=m\right) g_{n}(m) & =\mathbb{E}_{\mathbb{Q}_{n}}\left[1\{I=1\} \cdot 1\left\{\delta_{n}^{m} B^{H} \in A\right\} \mid M=m\right] g_{n}(m) \\
& =\sum_{\pi=+,-} \sum_{k=1}^{2^{n+m-1}} \mathbb{E}_{\mathbb{Q}_{n}^{(m, k, \pi)}}\left[1\{I=1\} \cdot 1\left\{\delta_{n}^{m} B^{H} \in A\right\}\right] \cdot \frac{g_{n}(m)}{2^{n+m}} .
\end{aligned}
$$

For each term in the summation in (31), as

$$
\begin{aligned}
& \mathbb{E}_{\mathbb{Q}_{n}^{(m, k, \pi)}}\left[1\{I=1\} \mid \boldsymbol{B}_{n+m}^{H}\right] \\
& =\mathbb{E}_{\mathbb{Q}_{n}^{(m, k, \pi)}}\left[1\left\{\left\{\pi \cdot \boldsymbol{\beta}^{\top} \boldsymbol{\alpha}_{n}(m, k)>\rho \ell_{n+m}\right\} \cap\left\{\cap_{j=1}^{m-1}\left[\mathcal{C}_{n}(j)\right]^{c}\right\}\right\} \cdot R_{n+m}^{-1} \cdot \Theta_{n}^{\pi}(m, k) \mid \boldsymbol{B}_{n+m}^{H}\right],
\end{aligned}
$$

then we have

$$
\begin{aligned}
& \mathbb{E}_{\mathbb{Q}_{n}^{(m, k, \pi)}}\left[1\{I=1\} \cdot 1\left\{\delta_{n}^{m} B^{H} \in A\right\}\right] \frac{g_{n}(m)}{2^{n+m}} \\
= & \mathbb{E}_{\mathbb{Q}_{n}^{(m, k, \pi)}}\left[1\left\{\delta_{n}^{m} B^{H} \in A\right\} \cdot 1\left\{\left\{\pi \cdot \boldsymbol{\beta}^{\top} \boldsymbol{\alpha}_{n}(m, k)>\rho \ell_{n+m}\right\} \cap\left\{\cap_{j=1}^{m-1}\left[\mathcal{C}_{n}(j)\right]^{c}\right\}\right\} \cdot R_{n+m}^{-1} \cdot \Theta_{n}^{\pi}(m, k)\right] \frac{g_{n}(m)}{2^{n+m}} .
\end{aligned}
$$

Comparing (30) with (32), we have shown that the numerator in (27) is equal to the numerator in (28).

Theorem 5, together with Theorem 6 , justifies the correctness of our algorithm.

4.3. Computational complexity analysis In this section, we analyze the computational complexity ${ }^{1}$ of our algorithm. The first key component in our algorithm is to find the last recordbreaker and the associated path of the fBM. Once we have found the last record-breaker, to achieve an $\epsilon$ error bound, the complexity of the refinement step is bounded by $O\left(N(\epsilon) 2^{N(\epsilon)}\right)$. Note that the $O\left(N(\epsilon) 2^{N(\epsilon)}\right)$ complexity is nontrivial. We provide more details about the actual construction that allows us to achieve this complexity later in this section.

We use $\bar{N}$ to denote the last dyadic level we need to generate in order to determine the level of the last record-breaker. Note that in our algorithm, in order to apply the change-of-measure to propose the next record breaking level, we need to refine the dyadic approximation until the BCE condition is satisfied. Thus, $\bar{N}$ can be larger than $N$. We denote by $C_{\bar{N}}$ the associated computational complexity. The following theorem establishes that $C_{\bar{N}}$ has finite expectation.

TheOREm 7. For the cost of finding the last record-breaker in Algorithm 2, $C_{\bar{N}}$, we have

$$
\mathbb{E}\left[C_{\bar{N}}\right]<\infty
$$

${ }^{1}$ We refer to the computational complexity as the total number of uniform random variables we need to generate and the number of basic calculations. For example, the Cholesky decomposition of an $n \times n$ convariance matrix has a computational complexity of $O\left(n^{3}\right)$. 
Proof. In our algorithm, the computational cost comes from two main procedures. The first is refining the dyadic level at which the $\mathrm{BCE}$ condition is satisfied. The second is finding the next record-breaker or claiming that there is no more record-breaker given that the BCE condition holds. In what follows, for simplicity, we use $C$ to denote a generic constant, which may differ from line to line.

We first derive a bound for $\bar{N}$. Recall that $N$ is the level of the last record-breaker. We also recall that $\mathcal{N}_{\mathcal{E}}$ is the last level at which the BCE condition is violated. Note that we only need to check whether the BCE condition is violated at a specific level $n$ in Algorithm 3. Thus, if $\mathcal{N}_{\mathcal{E}}+1 \leq N$, the last level needed to find the last record-breaker is just $N$, i.e., $\bar{N}=N$. If $\mathcal{N}_{\mathcal{E}}+1>N$, we can stop the first time when BCE condition is satisfied after $N$, which is upper bounded by $\mathcal{N}_{\mathcal{E}}+1$. Therefore,

$$
\bar{N} \leq \max \left\{N, \mathcal{N}_{\mathcal{E}}+1\right\} .
$$

Secondly, we analyze the complexity of checking the BCE condition. Based on Algorithm 5, we need to calculate the conditional expectation until level $n+M_{n}=O\left(n+\log _{2}\left(\gamma_{n}\right)\right)$, whose computational complexity is upper bounded by $C 2^{3 n} \gamma_{n}^{3}$. Note that the BCE condition need to be checked at each refinement level. Thus the total complexity of checking the BCE condition is upper bounded by

$$
C \sum_{j=1}^{\mathcal{N}_{\mathcal{E}}+1} 2^{3 j} \gamma_{j}^{3}
$$

Thirdly, if the BCE condition is satisfied at level $n$, we shall apply the change-of-measure to propose a record-breaking path, i.e., call Algorithm 4. The computational complexity of the change-of-measure is upper bounded by

$$
C \sum_{m=1}^{\infty} g_{n}(m) \cdot 2^{3(n+m)} \leq C Z_{n}^{-1} \cdot \sum_{m=1}^{\infty} 2^{4(n+m)} \cdot \exp \left\{-\rho^{2} / 8 \cdot 2^{2(n+m) \delta}\right\} \leq C 2^{3 n} .
$$

We apply the change-of-measure at most $\bar{N}$ times and the last time we apply the change-of-measure is at level $\bar{N}$. Thus, the total complexity of the applying the change-of-measure is upper bounded by $C \bar{N} 2^{3 \bar{N}}$.

Combining the second part and the third part together, we have

$$
C_{\bar{N}} \leq C \sum_{j=1}^{\mathcal{N}_{\mathcal{E}}+1} 2^{3 j} \gamma_{j}^{3}+C \bar{N} 2^{3 \bar{N}}
$$

As $N$ and $\mathcal{N}_{\mathcal{E}}$ have finite moment generating functions, from (33), $\bar{N}$ also has a finite moment generating function. Then, $\mathbb{E}\left[\bar{N} 2^{3 \bar{N}}\right]<\infty$. We next show that the first term in (34) also has finite expectation.

$$
\begin{aligned}
\mathbb{E}\left[\sum_{j=1}^{\mathcal{N}_{\mathcal{E}}+1} 2^{3 j} \gamma_{j}^{3}\right] & =\mathbb{E}\left[\sum_{j=1}^{\infty} 2^{3 j} \gamma_{j}^{3} \cdot 1\left\{j \leq \mathcal{N}_{\mathcal{E}}+1\right\}\right] \\
& =\sum_{j=1}^{\infty} 2^{3 j} \mathbb{E}\left[\gamma_{j}^{3} \cdot 1\left\{j \leq \mathcal{N}_{\mathcal{E}}+1\right\}\right] \text { by Fubini's Theorem } \\
& \leq \sum_{j=1}^{\infty} 2^{3 j} \mathbb{E}\left[\gamma_{j}^{6}\right]^{1 / 2} \mathbb{P}\left(j \leq \mathcal{N}_{\mathcal{E}}+1\right)^{1 / 2} \text { by Cauchy-Schwartz inequality. }
\end{aligned}
$$


Recall that $\gamma_{n}$ is the maximal absolute value of the entries in the vector $\boldsymbol{\Sigma}_{n}^{-1} \boldsymbol{B}_{n}^{H}$, which follows multivariate normal distribution with mean $\mathbf{0}$ and covariance matrix $\boldsymbol{\Sigma}_{n}^{-1}$. Thus,

$$
\mathbb{E}\left[\gamma_{j}^{6}\right] \leq C \cdot 2^{j} \cdot 2^{6 H j}=C \cdot 2^{(1+6 H) j} .
$$

Then, similar to the proof of Theorem 3 , using the decay rate of $\mathbb{P}\left(\mathcal{N}_{\mathcal{E}} \geq j\right)$ proved in Lemma 6 , we obtain

$$
\sum_{j=1}^{\infty} 2^{3 j} \mathbb{E}\left[\gamma_{j}^{6}\right]^{1 / 2} \mathbb{P}\left(j \leq \mathcal{N}_{\mathcal{E}}+1\right)^{1 / 2}<\infty
$$

Hence, $\mathbb{E}\left[C_{\bar{N}}\right]<\infty$, i.e., our algorithm has finite expected computational complexity.

In our algorithm, once we have generated the fBM up to level $\bar{N}$, we can claim that we find the last record-breaker. To achieve the desired accuracy $\epsilon$, if $N(\epsilon)>\bar{N}$, we need to refine the dyadic approximation until the truncation level $N(\epsilon)$, conditioning on the event that the record will not be broken beyond $\bar{N}$. Note that $\bar{N}$ does not depend on $\epsilon$, and by Theorem $7, \mathbb{E}\left[C_{\bar{N}}\right]<\infty$. On the other hand, $N(\epsilon) \rightarrow \infty$ as $\epsilon \rightarrow 0$ (see (4)). Thus, $N(\epsilon)>\bar{N}$ eventually almost surely as $\epsilon \rightarrow 0$. To sample the fBM at the augmented time points $D_{N(\epsilon)} \backslash D_{\bar{N}}$ conditional on $\boldsymbol{B}_{\bar{N}}^{H}$, we next introduce a recursive construction that has complexity $O\left(2^{N(\epsilon)} \log \left(2^{N(\epsilon)}\right)\right)$. We comment that if we use Cholesky decomposition for this refinement step, the complexity is $O\left(2^{3 N(\epsilon)}\right)$. The recursive construction we employ here is based on the Gaussian bridge idea developed in Sottinen and Yazigi (2014). We summarize the main idea in the following lemma.

Lemma 8. Let $\left\{X_{t}\right\}_{t \geq 0}$ be a Gaussian process with covariance function $r(s, t)$. Then the distribution of $X_{t}$ conditional on that $X_{t_{k}}=y_{k}, k=1, \cdots, n$, is same as that of $X_{t}^{n}$, which is defined recursively as

$$
X_{t}^{0}=X_{t} \text { and } X_{t}^{k}=X_{t}^{k-1}-\frac{r_{k-1}\left(t, t_{k}\right)}{r_{k-1}\left(t_{k}, t_{k}\right)} \cdot\left(X_{t_{k}}^{k-1}-y_{k}\right), k=1, \cdots, n
$$

where

$$
r_{0}(s, t)=r(s, t) \text { and } r_{k}(s, t)=r_{k-1}(s, t)-\frac{r_{k-1}\left(s, t_{k}\right) \cdot r_{k-1}\left(t_{k}, t\right)}{r_{k-1}\left(t_{k}, t_{k}\right)}, k=1, \cdots, n
$$

In our case, we first sample $\boldsymbol{B}_{N(\epsilon)}^{H}$ without taking the conditioning using Davies-Harte method (Davies and Harte, 1987), which has complexity $O\left(2^{N(\epsilon)} \log \left(2^{N(\epsilon)}\right)\right.$ ). Then, for each $t \in D_{N(\epsilon)} \backslash D_{\bar{N}}$, we use the recursion in Lemma 8 to calculate the value of fBM. This has complexity $O\left(2^{3 \bar{N}}\right)$ as we are conditioning on $2^{\bar{N}}$ points of the Gaussian process. Thus, the total complexity can be upper bounded by $C 2^{N(\epsilon)} \log \left(2^{N(\epsilon)}\right)+C 2^{3 \bar{N}} 2^{N(\epsilon)}$ From the definition of $N(\epsilon)$ in $(4)$, we have $2^{N(\epsilon)}=C \epsilon^{-1 /(H-\delta)}$. Thus, the expected complexity of the refinement step is upper bounded by

$$
C \mathbb{E}\left[2^{N(\epsilon)} \log \left(2^{N(\epsilon)}\right)+2^{3 \bar{N}} 2^{N(\epsilon)}\right]=O\left(\epsilon^{-1 /(H-\delta)}\right) .
$$

Combining this with the complexity of finding the last record-breaker, the expected complexity of our algorithm to achieve a strong error bound $\epsilon$ is upper bounded by

$$
\mathbb{E}\left[C_{\bar{N}}\right]+O\left(2^{N(\epsilon)} \log \left(2^{N(\epsilon)}\right)\right)=O\left(\epsilon^{-1 /(H-\delta)}\right) \text { for any } \delta>0 .
$$

Lastly, we comment that Kühn and Linde (2002) show among a fairly general class of approximations, the optimal complexity is $\Theta(n(\epsilon))$, where $n(\epsilon)$ is the solution of equation $n^{-H} \sqrt{\log (n)}=\epsilon$. By simple calculation, we can show that $C_{1} \epsilon^{-1 / H} \leq n(\epsilon) \leq C_{2} \epsilon^{-1 / H} \cdot \log (1 / \epsilon)^{1 /(2 H)}$ for some constants $C_{1}, C_{2}>0$. As $\delta$ can be made arbitrary small, our algorithm is near optimal. 
5. $\epsilon$-Strong simulation of stochastic differential equation driven by fBM In this section, we extend the $\epsilon$-strong simulation algorithm for fBM to stochastic differential equations (SDEs) driven by fBM with $H>1 / 2$ via rough path theory. Consider a $d$-dimensional SDE

$$
\mathrm{d} \boldsymbol{Y}(t)=\boldsymbol{\mu}(\boldsymbol{Y}(t)) \mathrm{d} t+\boldsymbol{\sigma}(\boldsymbol{Y}(t)) \mathrm{d} \boldsymbol{B}^{H}(t), \boldsymbol{Y}(0)=\boldsymbol{y}(0),
$$

where $\boldsymbol{B}^{H}$ is a $d^{\prime}$-dimensional fBM (each component is an independent standard one-dimensional $\mathrm{fBM}), \boldsymbol{\mu}(\cdot): \mathbb{R}^{d} \rightarrow \mathbb{R}^{d}$ and $\boldsymbol{\sigma}(\cdot): \mathbb{R}^{d} \rightarrow \mathbb{R}^{d \times d^{\prime}}$ are vector fields corresponding to the drift and the volatility, respectively. For any fixed $\epsilon>0$, our goal is to construct a probability space, supporting both $\boldsymbol{Y}$ and a fully simulatable path $\hat{\boldsymbol{Y}}_{\epsilon}$ such that

$$
\sup _{t \in[0,1]}\left\|\boldsymbol{Y}(t)-\hat{\boldsymbol{Y}}_{\epsilon}(t)\right\| \leq \epsilon, \quad \text { a.s. }
$$

The construction of $\hat{\boldsymbol{Y}}_{\epsilon}(t)$ builds on our ability to estimate the driving fBM and its corresponding $\alpha$-Hölder norm. In particular, for any $1 / 2<\alpha<H$, the sample path of fBM is $\alpha$-Hölder continuous almost surely. In this case, by the rough path theory, the solution of SDE (35) can be defined path by path, and the mapping from $\boldsymbol{B}^{H}$ to $\boldsymbol{Y}$ is continuous under the $\alpha$-Hölder norm (Lyons, 1998). Therefore, if we can control the error of the simulated driving signals, by continuous mapping type of argument, we can also control the error of the simulated SDEs.

In what follows, we lay out the main idea of our algorithmic development. The theoretical foundation is provide in Section 5.1.

The construction of the approximated solution is based on simple Euler scheme. For dyadic discretization $D_{n}$, we define $\boldsymbol{Y}_{n}\left(t_{k}^{n}\right)$ via the recursion

$$
\begin{aligned}
\boldsymbol{Y}_{n}\left(t_{0}^{n}\right) & =0 \\
\boldsymbol{Y}_{n}\left(t_{k+1}^{n}\right) & =\boldsymbol{Y}^{(n)}\left(t_{k}^{n}\right)+\boldsymbol{\mu}\left(\boldsymbol{Y}_{n}\left(t_{k}^{n}\right)\right) \cdot \Delta_{n}+\boldsymbol{\sigma}\left(\boldsymbol{Y}_{n}\left(t_{k}^{n}\right)\right) \cdot\left(\boldsymbol{B}^{H}\left(t_{k+1}^{n}\right)-\boldsymbol{B}^{H}\left(t_{k}^{n}\right)\right), k=0,1, \ldots, 2^{n} .
\end{aligned}
$$

We then construct the whole path $\boldsymbol{Y}_{n}(t)$ via piecewise-constant interpolation,

$$
\boldsymbol{Y}_{n}(t)=\boldsymbol{Y}_{n}\left(t_{k}^{n}\right), t \in\left[t_{k}^{n}, t_{k+1}^{n}\right) .
$$

The challenge here is to choose an appropriate discretization level $N_{Y}(\epsilon)$, such that

$$
\left\|\boldsymbol{Y}_{N_{Y}(\epsilon)}-\boldsymbol{Y}\right\|_{\infty} \leq \epsilon \quad \text { a.s. }
$$

In Theorem 9 below, we establish that

$$
\left\|\boldsymbol{Y}_{n}-\boldsymbol{Y}\right\|_{\infty} \leq G \cdot \Delta_{n}^{2 \alpha-1},
$$

where $G$ can be characterized explicitly and is an increasing function with respect to the $\alpha$-Hölder norm of $\boldsymbol{B}^{H}$. Therefore, we shall first use the $\epsilon$-strong simulation algorithm we developed in Section 4 to find an upper bound for $\left\|\boldsymbol{B}^{H}\right\|_{\alpha}$. We can then upper bound $G$ by $\bar{G}$, and set

$$
N_{Y}(\epsilon)=\left\lceil\log _{2}\left(\epsilon^{-1} \bar{G} /(2 \alpha-1)\right)\right\rceil .
$$

The actual algorithm is summarized in Algorithm 6. Note that to achieve the accuracy of $\epsilon$, the expected computational complexity of Algorithm 6 is $O\left(\epsilon^{-1 /(2 \alpha-1)}\right)$.

We next provide two remarks about some extensions of our algorithm.

REMARK 3. For the efficiency of the algorithm, if we use the Euler scheme, then $\left\|\boldsymbol{Y}_{n}-\boldsymbol{Y}\right\|_{\infty} \leq$ $G \cdot \Delta_{n}^{2 \alpha-1}$ is near the optimal rate of convergence. If we use higher order approximation, we can achieve better convergence rate. For example, if we use the Milstein scheme, then we expect $\left\|\boldsymbol{Y}_{n}-\boldsymbol{Y}\right\|_{\infty} \leq C \cdot \Delta_{n}^{3 \alpha-1}$ for some $C>0$ (Davie, 2008). However, implementing these higher order schemes requires us to compute/estimate the iterated integrals of fBM, which is still largely an open problem. 


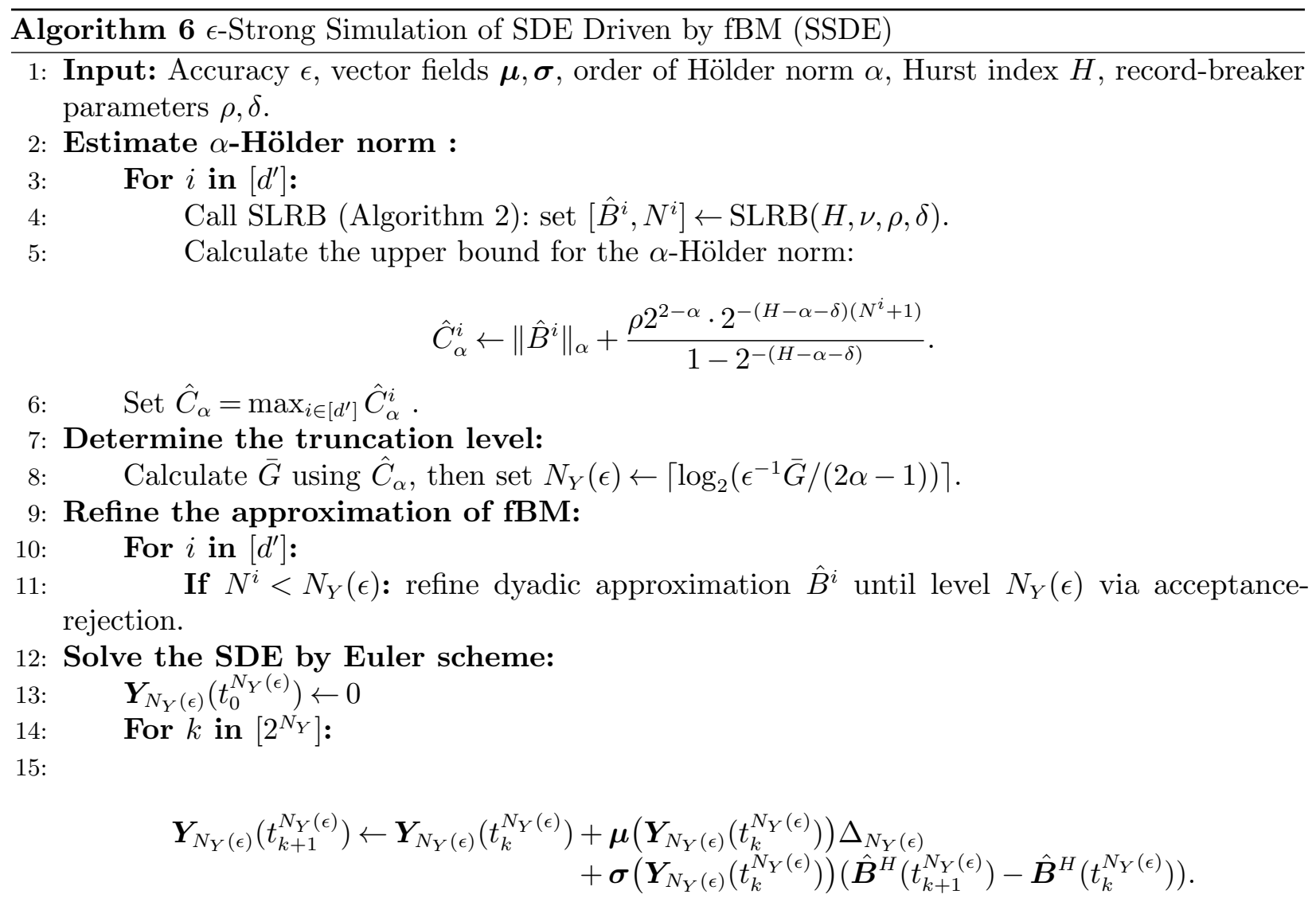

16: Output: Return $\boldsymbol{Y}_{N_{Y}(\epsilon)}(t)$, the piecewise constant interpolation of $\left\{\boldsymbol{Y}_{N_{Y}(\epsilon)}\left(t_{k}^{N_{Y}(\epsilon)}\right)\right\}_{k=0, \cdots, 2^{N_{Y}(\epsilon)}}$.

REMARK 4. For $H<1 / 2$, to define the SDE in a path-by-path sense and to apply continuous mapping argument, higher order iterated integrals of fBM need to be specified (Davie, 2008). In particular, we need to lift the fBM up to a rough path. Coutin and Qian (2002) construct a geometric rough path associated with fBM with Hurst index $H>1 / 4$, and develop a Skohorod integral representation of the geometric rough path. More recently, Nualart and Tindel (2011) develop a construction of the rough path above fBM using Volterra's representation for any $H \in(0,1)$. The key challenge remains as how to estimate the iterated integrals of fBM efficiently.

5.1. Rate of convergence of Euler scheme In this section, we present the details of the rate of convergence of Euler scheme for fBM driven SDEs. This result is an extension of Lejay (2010). In particular, we explicitly characterize the constant term in front of $\Delta_{n}^{2 \alpha-1}$ for the Euler scheme at dyadic level $n$. This is important for our algorithmic development, as we need to know $G$ in (36) to find the required discretization level $N_{Y}(\epsilon)$.

We first introduce a few notations to simplify the exposition. Consider the following multidimensional controlled differential equation system $\boldsymbol{y}$ driven by vector-valued signal $\boldsymbol{x}$

$$
\left[\begin{array}{c}
\mathrm{d} y^{1}(t) \\
\mathrm{d} y^{2}(t) \\
\vdots \\
\mathrm{d} y^{d}(t)
\end{array}\right]=\left[\begin{array}{cccc}
f_{11}(\boldsymbol{y}(t)) & f_{12}(\boldsymbol{y}(t)) & \cdots & f_{1 h}(\boldsymbol{y}(t)) \\
f_{21}(\boldsymbol{y}(t)) & f_{22}(\boldsymbol{y}(t)) & \cdots & f_{2 h}(\boldsymbol{y}(t)) \\
\vdots & \vdots & & \vdots \\
f_{d 1}(\boldsymbol{y}(t)) & f_{d 2}(\boldsymbol{y}(t)) & \cdots & f_{d h}(\boldsymbol{y}(t))
\end{array}\right] \cdot\left[\begin{array}{c}
\mathrm{d} x^{1}(t) \\
\mathrm{d} x^{2}(t) \\
\vdots \\
\mathrm{d} x^{h}(t)
\end{array}\right], \quad\left[\begin{array}{c}
y^{1}(0) \\
y^{2}(0) \\
\vdots \\
y^{d}(0)
\end{array}\right]=\left[\begin{array}{c}
y_{0}^{1} \\
y_{0}^{2} \\
\vdots \\
y_{0}^{d}
\end{array}\right],
$$


where $\boldsymbol{y}(t)=\left[y^{1}(t), \cdots, y^{d}(t)\right]^{\top}$ and $\boldsymbol{x}(t)=\left[x^{1}(t), \cdots, x^{h}(t)\right]^{\top}$. It is easy to see that SDE (35) can be written in form of (37). We only need to set

$$
\boldsymbol{f}(\cdot)=[\boldsymbol{\mu}(\cdot), \boldsymbol{\sigma}(\cdot)] \text { and } \mathrm{d} \boldsymbol{x}(t)=\left[\begin{array}{c}
\mathrm{d} t \\
\mathrm{~d} \boldsymbol{B}^{H}(t)
\end{array}\right],
$$

and then dimension $h=d^{\prime}+1$. From now on, all of our derivation will be based on the notations in (37). In the following of this section, we use $\boldsymbol{f}$ to denote the matrix $\left[f_{i j}\right]_{d \times h}$. Furthermore, we assume that $\boldsymbol{x}$ is $\alpha$-Hölder continuous with $1 / 2<\alpha \leq 1$, which is to say, $\|\boldsymbol{x}(t)-\boldsymbol{x}(s)\| \leq C_{\alpha}|t-s|^{\alpha}$ for some $C_{\alpha} \in(0, \infty)$. In what follows, we use $C_{\alpha}$ to denote a computable upper bound for the $\alpha$-Hölder norm of $\boldsymbol{x}$.

The solution to equation (37) is formally defined in terms of the Young integral. Formally, we have the following definition.

Definition 2. We say that $\boldsymbol{y}$ is a solution of equation (37) if for all $t \in[0,1]$,

$$
\boldsymbol{y}(t)=\boldsymbol{y}_{0}+\int_{0}^{t} \boldsymbol{f}(\boldsymbol{y}(s)) \mathrm{d} \boldsymbol{x}(s),
$$

where the integration is interpreted as Young integral.

In what follows, we first provide a brief introduction to Young integral. Then, we quantify the error of the Euler scheme.

Young integral is an extension of Riemann-Stieltjes integral for paths with finite $p$-variation for $1 \geq p<2$, but potentially infinite total variation. We say a continuous path $\boldsymbol{u} \in \mathbb{R}^{h}$ defined on $[0,1]$ has finite $p$-variation if

$$
\sup _{\Pi} \sum_{t_{i} \in \Pi}\left\|\boldsymbol{u}\left(t_{i+1}\right)-\boldsymbol{u}\left(t_{i}\right)\right\|^{p}<\infty
$$

where $\Pi=\left\{t_{i}\right\}_{i \geq 0}$ is a set of finite partitions of $[0,1]$.

Definition 3. (Young integral) Let $\boldsymbol{u}$ and $\boldsymbol{v}$ be continuous paths on $[0,1]$ with finite $p$-variation and $q$-variation respectively, such that $1 / p+1 / q>1$. Then the limit of Riemann sum as the mesh of the partition $|\Pi|$ goes to zero

$$
\lim _{|\Pi| \rightarrow 0} \sum_{t_{i} \in \Pi} \boldsymbol{v}\left(t_{i}\right) \cdot\left(\boldsymbol{u}\left(t_{i+1}\right)-\boldsymbol{u}\left(t_{i}\right)\right)
$$

exists and is unique. We use $\int_{0}^{1} \boldsymbol{v}(s) \mathrm{d} \boldsymbol{u}(s)$, to denote this limit and call it the Young integral of $\boldsymbol{v}$ with respect to $\boldsymbol{u}$.

A special case of finite $p$-variation path is $\alpha$-Hölder continuous path. In particular, if $\boldsymbol{u}$ is $\alpha$-Hölder continuous, i.e. $\|\boldsymbol{u}(s)-\boldsymbol{u}(t)\| \leq\|\boldsymbol{u}\|_{\alpha} \cdot|s-t|^{\alpha}$ for some $\|\boldsymbol{u}\|_{\alpha} \in(0, \infty)$, then $u$ has finite $p$-variation with $p=1 / \alpha$. In this case, we have the following Young-Lóeve estimate (Lejay (2010)).

TheOrem 8. (Young-Lóeve estimate.) Assume that the integrator $u$ and the integrand $v$ are Hölder continuous of exponents $\alpha$ and $\beta$ with $\alpha+\beta>1$, respectively. Then for any $s, t \in[0,1]$,

$$
\left\|\int_{s}^{t} \boldsymbol{v}(r) \mathrm{d} \boldsymbol{u}(r)-\boldsymbol{v}(s)(\boldsymbol{u}(t)-\boldsymbol{u}(s))\right\| \leq K(\alpha+\beta) \cdot\|\boldsymbol{u}\|_{\alpha} \cdot\|\boldsymbol{v}\|_{\beta} \cdot|t-s|^{\alpha+\beta}
$$

where $K(\alpha+\beta)=\left(1+\sum_{n \geq 1} n^{-(\alpha+\beta)}\right) \cdot h$. Moreover, for any finite partition $\Pi_{s, t}$ of $[s, t]$,

$$
\left\|\sum_{t_{i} \in \Pi_{s, t}} \boldsymbol{v}\left(t_{i}\right)\left(\boldsymbol{u}\left(t_{i+1}\right)-\boldsymbol{u}\left(t_{i}\right)\right)-\boldsymbol{v}(s)(\boldsymbol{u}(t)-\boldsymbol{u}(s))\right\| \leq K(\alpha+\beta) \cdot\|\boldsymbol{u}\|_{\alpha} \cdot\|\boldsymbol{v}\|_{\beta} \cdot|t-s|^{\alpha+\beta},
$$


For dyadic discretization points $D_{n}=\left\{t_{k}^{n}\right\}_{k=0, \cdots, 2^{n}}$, we define

$$
\boldsymbol{y}_{n}\left(t_{k+1}^{n}\right)=\boldsymbol{y}_{n}\left(t_{k}^{n}\right)+\boldsymbol{f}\left(\boldsymbol{y}_{n}\left(t_{k}^{n}\right)\right) \cdot\left(\boldsymbol{x}\left(t_{k+1}^{n}\right)-\boldsymbol{x}\left(t_{k}^{n}\right)\right), k=0,1, \cdots, 2^{n}-1 .
$$

Based on the values $\left\{\boldsymbol{y}_{n}\left(t_{k}^{n}\right)\right\}_{k=0, \cdots, 2^{n}}$, we further construct a path over [0,1] via piecewise constant interpolation, i.e.

$$
\boldsymbol{y}_{n}(t)=\boldsymbol{y}_{n}\left(t_{k}^{n}\right), t \in\left[t_{k}^{n}, t_{k+1}^{n}\right) \text {. }
$$

We call $\boldsymbol{y}_{n}$ an approximated solution of level $n$ via Euler scheme. Our goal is to control the uniform norm between the approximated solution $\boldsymbol{y}_{n}$ and exact solution $\boldsymbol{y}$.

To ensure the existence of solution of controlled differential equation (37) and control the approximation error, we impose the following smoothness condition on the vector field $\boldsymbol{f}$. We define $\nabla \boldsymbol{f}$ as a $d \times h \times d$ tensor with the $(i, j, k)$ element $\nabla f_{i j k}=\partial f_{i j} / \partial y_{k}$. We also define $\nabla^{2} \boldsymbol{f}$ as a $d \times h \times d \times d$ tensor with the $(i, j, k, l)$ element $\partial^{2} f_{i j} / \partial y_{k} \partial y_{l}$. With a little abuse of notation, we denote

$$
\|\boldsymbol{f}\|=\sup _{\boldsymbol{y} \in \mathbb{R}^{d}} \max _{i j}\left|f_{i j}(\boldsymbol{y})\right|, \quad\|\nabla \boldsymbol{f}\|=\sup _{\boldsymbol{y} \in \mathbb{R}^{d}} \max _{i j k}\left|\frac{\partial f_{i j}(\boldsymbol{y})}{\partial y_{k}}\right|, \text { and }\left\|\nabla^{2} \boldsymbol{f}\right\|=\sup _{\boldsymbol{y} \in \mathbb{R}^{d}} \max _{i j k l}\left|\frac{\partial^{2} f_{i j}(\boldsymbol{y})}{\partial y_{k} \partial y_{l}}\right| .
$$

Assumption 1. We assume that $\boldsymbol{f}(\cdot)$ is twice continuously differentiable, and

$$
\max \left\{\|\boldsymbol{f}\|,\|\nabla \boldsymbol{f}\|,\left\|\nabla^{2} \boldsymbol{f}\right\|\right\}<\infty .
$$

We also define the following constants. Recall that $C_{\alpha}$ is a computable upper bound for the $\alpha$-Hölder norm of $\boldsymbol{x}$ and the function $K(\cdot)$ is defined in Theorem 8. Let

$$
\begin{aligned}
& G_{1}^{*}=2 h \cdot\left\lceil\left(2 d h C_{\alpha} K(2 \alpha)\|\nabla \boldsymbol{f}\|\right)^{1 / \alpha}\right\rceil^{1-\alpha} \cdot\|\boldsymbol{f}\| \cdot C_{\alpha}, \quad G_{2}^{*}=d h \cdot K(2 \alpha) \cdot\|\nabla \boldsymbol{f}\| \cdot C_{\alpha} \cdot G_{1}^{*}, \\
& L=4 /\left(1-2^{1-2 \alpha}\right) \cdot\left(h C_{\alpha}\right)^{2}\|\nabla \boldsymbol{f}\| \cdot\|\boldsymbol{f}\|, \quad \omega=\left(h\|\boldsymbol{f}\| C_{\alpha} / L\right)^{1 / \alpha}, \\
& G_{1}=\left(L+h\|\boldsymbol{f}\| C_{\alpha}\right) \cdot\left(1+\omega^{-1}\right), \quad G_{2}=\max \left\{\left(2 \omega^{-\alpha}+\omega^{-1-\alpha}\right) \cdot\left(L+h\|\boldsymbol{f}\| C_{\alpha}\right), L\right\} .
\end{aligned}
$$

In addition, we define a sequence of constants $\left\{\Gamma_{k}\right\}_{k \geq 1}$ and $\left\{\Upsilon_{k}\right\}_{k \geq 1}$ via recursion

$$
\begin{aligned}
& \Gamma_{1}=2 G_{2}^{*}, \Upsilon_{1}=(4 \zeta)^{-1} \cdot \Gamma_{1} \\
& \Gamma_{k}=2\left(G_{2}^{*}+v \cdot \Upsilon_{k-1}\right), \Upsilon_{k}=(4 \zeta)^{-1} \cdot \Gamma_{k}+\Upsilon_{k-1}, k \geq 2
\end{aligned}
$$

where

$$
\begin{aligned}
& \zeta=h \cdot K(2 \alpha) \cdot C_{\alpha} \cdot\left(d \cdot\|\nabla \boldsymbol{f}\|+d^{2} \cdot\left\|\nabla^{2} \boldsymbol{f}\right\| \cdot\left(G_{1}^{*}+G_{1}\right)\right) \\
& v=C_{\alpha} \cdot\left(d^{2} h \cdot K(2 \alpha) \cdot\left\|\nabla^{2} \boldsymbol{f}\right\| \cdot\left(G_{1}^{*}+G_{1}\right)+d \cdot\|\nabla \boldsymbol{f}\|\right) .
\end{aligned}
$$

Under Assumption 1, the general theory of Young integral equation ensures the existence and uniqueness for the solution of equation (37). The following theorem characterizes the rate of convergence of the Euler scheme under the uniform norm and it provides the theoretical foundation for Algorithm 6. Its proof can be found in Appendix C.

TheOREM 9. Under Assumption 1,

$$
\left\|\boldsymbol{y}_{n}-\boldsymbol{y}\right\|_{\infty} \leq G \cdot \Delta_{n}^{2 \alpha-1}:=\left(\Upsilon_{\left\lceil(4 \zeta)^{1 / \alpha}\right\rceil}+G_{1}^{*}\right) \cdot \Delta_{n}^{2 \alpha-1} .
$$

REMARK 5. By the definition of $G$, it is easy to see that $G$ is an increasing function with respect to $C_{\alpha}$. 


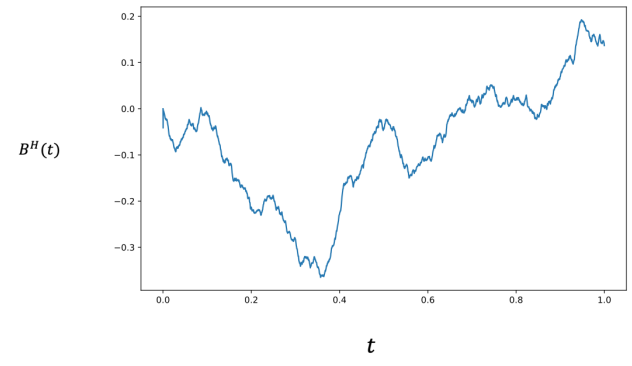

(a)

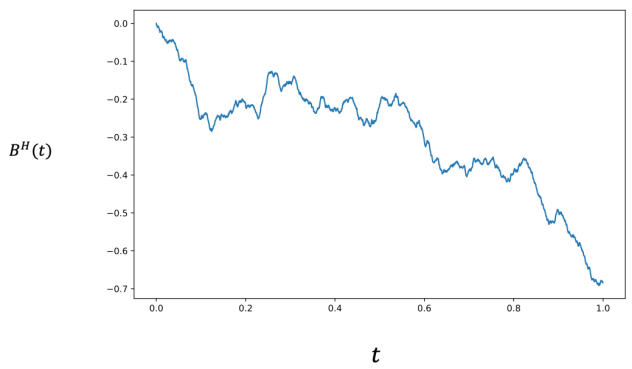

(b)

Figure 1. Two realizations of the $\epsilon$-strong simulation of fBM with $H=0.8$. We set $\epsilon=0.1, \rho=5, \delta=0.1$.

6. Numerical experiments In this section, we conduct some numerical experiments as a sanity check of the correctness of our algorithm. We also provide some discussions about how to choose the hyper-parameters $\rho$ and $\delta$ in actual implementations.

Figure 1 displays two realizations of a fBM with $H=0.8$ using Algorithm 1 with $\epsilon=0.1$.

We next study the effect of different values of $\rho$ and $\delta$. Recall that the record-breaking threshold takes the form $\rho \Delta_{n}^{H-\delta}$ for level $n$. $\delta$ determines the asymptotic decay rate of the bound and $\rho$ determines the scale of the bound. Thus, we would want both to be as small as possible when only considering truncation level, $N(\epsilon)$. Now, when also taking into account the time of the last record-breaker, $N$, we notice that larger values of $\rho$ and $\delta$ result in larger thresholds, under which, the records are less likely to be broken. These in turn lead to a smaller value of $N$. We also note that larger values of $\rho$ and $\delta$ lead to smaller stating level $N^{*}(\rho, \delta)$ in Algorithm 2. In the asymptotic sense, as $N$ and $N^{*}(\rho, \delta)$ do not depends on $\epsilon$, the values of $N$ and $N^{*}(\rho, \delta)$ do not affect the asymptotic behavior of our algorithms. This indicates that in theory, we should set $\rho$ and $\delta$ as small as possible. However, in practice, we do care about the "cost" of sampling $N$. Thus, in actual implementations, we will tune $\rho$ and $\delta$ to balance $N^{*}(\rho, \delta)$ and $N(\epsilon)$. Table 1 and 2 show the truncation level, the starting level, and the average level of the last record-breaker, $\left(N(\epsilon), N^{*}(\rho, \delta), \mathbb{E}[N]\right)$, for different choices of $\rho$ and $\delta$. Table 1 is for fBM with $H=0.8$ and Table 2 is for fBM with $H=0.45$. We make two observations from the tables. First, the level of the last record-breaker, $N$, is quite sensitive to our choice of $\rho$. For reasonably large values of $\rho$, e.g. $\rho \geq 2.5$, the record breaker rarely happens beyond level $n=1$. On the other hand, as we have discussed above, smaller values of $\rho$ lead to smaller values of truncation level $N(\epsilon)$. Second, the starting level $N^{*}(\rho, \delta)$ can be unreasonably large if $\rho$ and $\delta$ are not properly chosen. To sum up, as record-breaking is a rare event ${ }^{2}$, the benefits of shrinking the truncation level by choosing small $\rho$ and $\delta$ is very appealing. However, in practice, small values of $\rho$ and $\delta$ may lead to a large value of $N^{*}(\rho, \delta)$.

\begin{tabular}{|c|c|c|c|}
\hline$\delta$ & $\rho$ & 2.5 & 5 \\
\hline 0.1 & $(7,38,14)$ & $(9,21,1)$ & $(11,1,1)$ \\
\hline 0.2 & $(9,16,6)$ & $(11,6,1)$ & $(12,1,1)$ \\
\hline
\end{tabular}

TABle 1. $\left(N(\epsilon), N^{*}(\rho, \delta), \mathbb{E}[N]\right)$ under different choices of $\rho$ and $\delta$ when $H=0.8, \epsilon=0.1$.

\footnotetext{
${ }^{2}$ The corresponding probability decays double exponentially fast in $n$.
} 


\begin{tabular}{|c|c|c|c|}
\hline$\delta$ & 1 & 2.5 & 5 \\
\hline 0.1 & $(16,38,15+)$ & $(20,21,1)$ & $(23,1,1)$ \\
\hline 0.2 & $(24,16,8)$ & $(30,6,1)$ & $(31,1,1)$ \\
\hline
\end{tabular}

TABle 2. $\left(N(\epsilon), N^{*}(\rho, \delta), \mathbb{E}[N]\right)$ under different choices of $\rho$ and $\delta$ when $H=0.45, \epsilon=0.1$. (15+ means even at level 15 we still see record-breaker happening.)

Acknowledgments. JD and YC is supported by NSF Grant DMS-1720433. HN is supported by the EPSRC under the program grant EP/S026347/1 and by the Alan Turing Institute under the EPSRC grant EP/N510129/1

\section{References}

Abry P, Sellan F (1996) The wavelet-based synthesis for fractional Brownian motion proposed by F. Sellan and Y. Meyer: Remarks and fast implementation. Applied and Computational Harmonic Analysis 3(4):377-383.

Ayache A, Taqqu M (2003) Rate optimality of wavelet series approximations of fractional Brownian motion. Journal of Fourier Analysis and Applications 9(5):465-494.

Beskos A, Peluchetti S, Roberts G (2012) $\varepsilon$-Strong simulation of the Brownian path. Bernoulli 18(4):1223-1248.

Beskos A, Roberts G (2005) Exact simulation of diffusions. The Annals of Applied Probability 15(4):2422-2444.

Blanchet J, Chen X (2015) Steady-state simulation for reflected Brownian motion and related networks. The Annals of Applied Probability 25(6):3209-3250.

Blanchet J, Chen X, Dong J (2017) $\varepsilon$-Strong simulation for multidimensional stochastic differential equations via rough path analysis. The Annals of Applied Probability 27(1):275-336.

Blanchet J, Dong J (2015) Perfect sampling for infinite server and loss systems. Advances in Applied Probability 47(3):761-786.

Blanchet J, Sigman K (2011) On exact sampling of stochastic perpetuities. Journal of Applied Probability 48(A):165-182.

Blanchet J, Zhang F (2017) Exact simulation for multivariate Itó diffusions. arXiv preprint arXiv:1706.05124.

Chen N, Huang Z (2013) Localization and exact simulation of Brownian motion-driven stochastic differential equations. Mathematics of Operations Research 38(3):591-616.

Coutin L, Qian Z (2002) Stochastic analysis, rough path analysis and fractional Brownian motions. Probability theory and related fields 122(1):108-140.

Davie A (2008) Differential equations driven by rough paths: an approach via discrete approximation. Applied Mathematics Research eXpress 2008.

Davies R, Harte D (1987) Tests for Hurst effect. Biometrika 74(1):95-101.

Dieker A (2004) Simulation of fractional Brownian motion. Ph.D. thesis, Masters Thesis, Department of Mathematical Sciences, University of Twente.

Dieker A, Mandjes M (2003) On spectral simulation of fractional Brownian motion. Probability in the Engineering and Informational Sciences 17(3):417-434.

Dietrich C, Newsam G (1997) Fast and exact simulation of stationary Gaussian processes through circulant embedding of the covariance matrix. SIAM Journal on Scientific Computing 18(4):10881107.

Durbin J (1960) The fitting of time-series models. Revue de l'Institut International de Statistique 233-244.

Dzhaparidze K, Zanten HV (2004) A series expansion of fractional Brownian motion. Probability theory and related fields 130(1):39-55. 
Giles M (2008) Multilevel Monte Carlo path simulation. Operations Research 56(3):607-617.

Glynn P (2016) Exact simulation vs exact estimation. Proceedings of the Winter Simulation Conference, 2016, 193-205.

Höfling F, Franosch T (2013) Anomalous transport in the crowded world of biological cells. Reports on Progress in Physics 76(4):046602.

Huber M (2016) Nearly optimal Bernoulli factories for linear functions. Combinatorics, Probability and Computing 25(4):577-591.

Kühn T, Linde W (2002) Optimal series representation of fractional Brownian sheets. Bernoulli 8(5):669-696.

Laskin N (2000) Fractional market dynamics. Physica A: Statistical Mechanics and its Applications 287(3-4):482-492.

Lau W, A Erramilli JW, Willinger W (1995) Self-similar traffic generation: the random midpoint displacement algorithm and its properties. Proceedings IEEE International Conference on Communications ICC'95, volume 1, 466-472 (IEEE).

Lejay A (2010) Controlled differential equations as Young integrals: a simple approach. Journal of Differential Equations 249(8):1777-1798.

Levinson N (1946) The Wiener root mean square error criterion in filter design and prediction. Journal of Mathematics and Physics 25(1-4):261-278.

Liu Z, Blanchet J, Dieker A, Mikosch T (2019) On logrithmically optimal exact simulation of max-stable and related random fields on a compact set. Bernoulli 25(4A):2949-2981.

Lyons T (1998) Differential equations driven by rough signals. Revista Matemática Iberoamericana 14(2):215-310.

Meyer Y, Sellan F, Taqqu M (1999) Wavelets, generalized white noise and fractional integration: the synthesis of fractional Brownian motion. Journal of Fourier Analysis and Applications $5(5): 465-494$.

Norros I (1995) On the use of fractional Brownian motion in the theory of connectionless networks. IEEE Journal on selected areas in communications 13(6):953-962.

Norros I, Mannersalo P, Wang J (1999) Simulation of fractional Brownian motion with conditionalized random midpoint displacement. Advances in Performance Analysis 2(1):77-101.

Nualart D (2006) Fractional Brownian motion: stochastic calculus and applications. International Congress of Mathematicians, volume 3, 1541-1562 (Eur. Math. Soc.).

Nualart D, Tindel S (2011) A construction of the rough path above fractional Brownian motion using Volterra's representation. The Annals of Probability 39(3):1061-1096.

Pollock M, Johansen A, Roberts G (2016) On the exact and $\varepsilon$-strong simulation of (jump) diffusions. Bernoulli 22(2):794-856.

Rao C (1973) Linear statistical inference and its applications, volume 2 (Wiley New York).

Rhee C, Glynn P (2012) A new approach to unbiased estimation for SDE's. Proceedings of the Winter Simulation Conference, 2012, 17.

Sottinen T, Yazigi A (2014) Generalized Gaussian bridges. Stochastic Processes and Their Applications 124(9):3084-3105.

Wood A, Chan G (1994) Simulation of stationary Gaussian processes in $[0,1]^{d}$. Journal of computational and graphical statistics 3(4):409-432.

\section{Appendix A: Detailed Proofs for Theorems and Lemmas in Section 3}

\section{A.1. Proof of of the auxiliary lemmas for the proof of Theorem 1}

A.1.1. Proof of Lemma 1 According to the definitions of $a_{j}^{k}, c_{j}^{k}$ and $\boldsymbol{B}_{k}^{H}$, we have

$$
\begin{aligned}
\mathbb{V}_{k-1}\left(a_{j}^{k}-c_{j}^{k}\right)=\mathbb{V}\left(B^{H}\left(t_{2 j+1}^{k}\right) \mid \boldsymbol{B}_{k-1}^{H}\right) & =\mathbb{V}\left(B^{H}\left(t_{2 j+1}^{k}\right) \mid B^{H}\left(t_{0}^{k-1}\right), B^{H}\left(t_{1}^{k-1}\right), \cdots, B^{H}\left(t_{2^{k-1}}^{k-1}\right)\right) \\
& \leq \mathbb{V}\left(B^{H}\left(t_{2 j+1}^{k}\right) \mid B^{H}\left(t_{j}^{k-1}\right)\right) .
\end{aligned}
$$


where the inequality follows from the fact that for random variables $X, Y$ and $Z$, we have $\mathbb{E}[\mathbb{V}(X \mid Y, Z)] \leq \mathbb{E}[\mathbb{V}(X \mid Y)]$ and when the joint distribution of $(X, Y, Z)$ is multivariate Gaussian, the conditional variance $\mathbb{V}(X \mid Y, Z)$ and $\mathbb{V}(X \mid Y)$ are both constants.

The upper bound of conditional variance $\mathbb{V}\left(B^{H}\left(t_{2 j+1}^{k}\right) \mid B^{H}\left(t_{j}^{k-1}\right)\right)$ is based on the orthogonal bridge decomposition of Gaussian bridge (Sottinen and Yazigi (2014)), which is summarized in Lemma 8. Specifically, let $r(\cdot, \cdot)$ denote the covariance function of $B^{H}$, then the distribution of $B^{H}(t)$ conditional on $B^{H}\left(t_{j}^{k-1}\right)=y^{*}$ is same with that of

$$
B_{t_{j}^{k-1}, y^{*}}^{H}(t)=B^{H}(t)-\frac{r\left(t, t_{j}^{k-1}\right)}{r\left(t_{j}^{k-1}, t_{j}^{k-1}\right)} \cdot\left(B^{H}\left(t_{j}^{k-1}\right)-y^{*}\right) .
$$

By simple calculation, the covariance function of $B_{t_{j}^{k-1}, y^{*}}^{H}(t)$ is given by

$$
r_{t_{j}^{k-1}, y^{*}}(s, t)=r(s, t)-\frac{r\left(t, t_{j}^{k-1}\right) \cdot r\left(t_{j}^{k-1}, s\right)}{r\left(t_{j}^{k-1}, t_{j}^{k-1}\right)} .
$$

Recall that $\Delta_{k}=t_{2 j+1}^{k}-t_{j}^{k-1}=2^{-k}$, then we have

$$
\begin{aligned}
\mathbb{V}\left(B^{H}\left(t_{2 j+1}^{k}\right) \mid B^{H}\left(t_{j}^{k-1}\right)\right) & =r_{t_{j}^{k-1}, y^{*}}\left(t_{2 j+1}^{k}, t_{2 j+1}^{k}\right) \\
& =\left|t_{j}^{k-1}+\Delta_{k}\right|^{2 H}-1 / 4 \cdot\left(\left|t_{j}^{k-1}+\Delta_{k}\right|^{2 H}+\left|t_{j}^{k-1}\right|^{2 H}-\left|\Delta_{k}\right|^{2 H}\right)^{2} \cdot\left|t_{j}^{k-1}\right|^{-2 H} .
\end{aligned}
$$

In the next, we show that $\mathbb{V}\left(B^{H}\left(t_{2 j+1}^{k}\right) \mid B^{H}\left(t_{j}^{k-1}\right)\right)$ is no greater than $2 \cdot \Delta_{k}^{2 H}$, which is equivalent to

$$
|2 j+1|^{2 H}-1 / 4 \cdot\left(|2 j+1|^{2 H}+|2 j|^{2 H}-1\right)^{2} \cdot|2 j|^{-2 H} \leq 2,
$$

for arbitrary positive integers $j$. By calculation, the left-hand side of above inequality becomes

$$
\frac{1}{4} \cdot\left[2 \cdot|2 j+1|^{2 H}-\left(\frac{|2 j+1|^{4 H}}{|2 j|^{2 H}}+|2 j|^{2 H}\right)\right]-\frac{1}{4} \cdot|2 j|^{-2 H}+\frac{1}{2} \cdot\left(\frac{|2 j+1|^{2 H}}{|2 j|^{2 H}}+1\right) .
$$

Obviously, this quantity is no greater than 2. Thus, above all, we obtain

$$
\mathbb{V}_{k-1}\left(a_{j}^{k}-c_{j}^{k}\right) \leq \mathbb{V}\left(B^{H}\left(t_{2 j+1}^{k}\right) \mid B^{H}\left(t_{j}^{k-1}\right)\right) \leq 2 \cdot \Delta_{k}^{2 H}=2 \cdot 2^{-2 k H},
$$

which concludes the proof.

A.1.2. Proof of Lemma 2 Let $Y=\max _{1 \leq i \leq n}\left|X_{i}\right|$. For any $\eta>0$, by Jensen's inequality, we have

$$
\begin{aligned}
\exp \{\eta \mathbb{E}[Y]\} & \leq \mathbb{E}[\exp \{\eta Y\}]=\mathbb{E}\left[\max _{1 \leq i \leq n} \exp \left\{\eta\left|X_{i}\right|\right\}\right] \leq \sum_{i=1}^{n} \mathbb{E}\left[\exp \left\{\eta\left|X_{i}\right|\right\}\right] \\
& \leq \sum_{i=1}^{n} \mathbb{E}\left[\exp \left\{\eta X_{i}\right\}\right]+\mathbb{E}\left[\exp \left\{-\eta X_{i}\right\}\right] \leq 2 n \cdot \exp \left\{\eta^{2} \sigma^{2} / 2\right\}
\end{aligned}
$$

Hence, we obtain $\mathbb{E}[Y] \leq \log (2 n) / \eta+\eta \sigma^{2} / 2$ and the result follows by setting $\eta=\sqrt{2 \log (2 n)} / \sigma$. 
A.1.3. Proof of Lemma 3 In the following, we denote by

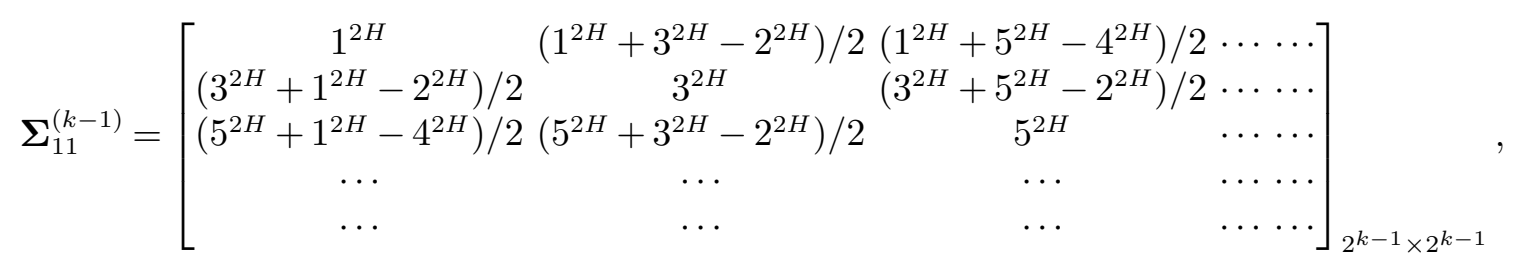

which is a $2^{k-1}$ by $2^{k-1}$ matrix with $(i, j)$-th entry $\left(|2 i-1|^{2 H}+|2 j-1|^{2 H}-|2 i-2 j|^{2 H}\right) / 2$. Recall the definition of $\boldsymbol{N}_{k-1}$ and $\boldsymbol{M}_{k-1}$, we have

$$
\begin{aligned}
& \left(\boldsymbol{N}_{k-1}-\boldsymbol{M}_{k-1}\right) \boldsymbol{\Sigma}_{22}^{(k-1)}\left(\boldsymbol{N}_{k-1}-\boldsymbol{M}_{k-1}\right)^{\top} \\
& =\boldsymbol{M}_{k-1} \boldsymbol{\Sigma}_{22}^{(k-1)} \boldsymbol{M}_{k-1}^{\top}-\boldsymbol{M}_{k-1}\left[\boldsymbol{\Sigma}_{12}^{(k-1)}\right]^{\top}-\boldsymbol{\Sigma}_{12}^{(k-1)} \boldsymbol{M}_{k-1}^{\top}+\boldsymbol{\Sigma}_{12}^{(k-1)} \cdot\left[\boldsymbol{\Sigma}_{22}^{(k-1)}\right]^{-1}\left[\boldsymbol{\Sigma}_{12}^{(k-1)}\right]^{\top} \\
& =\left(\boldsymbol{M}_{k-1} \boldsymbol{\Sigma}_{22}^{(k-1)} \boldsymbol{M}_{k-1}^{\top}-\boldsymbol{M}_{k-1}\left[\boldsymbol{\Sigma}_{12}^{(k-1)}\right]^{\top}-\boldsymbol{\Sigma}_{12}^{(k-1)} \boldsymbol{M}_{k-1}^{\top}+\boldsymbol{\Sigma}_{11}^{(k-1)}\right) \\
& \quad-\left(\boldsymbol{\Sigma}_{11}^{(k-1)}-\boldsymbol{\Sigma}_{12}^{(k-1)} \cdot\left[\boldsymbol{\Sigma}_{22}^{(k-1)}\right]^{-1}\left[\boldsymbol{\Sigma}_{12}^{(k-1)}\right]^{\top}\right) .
\end{aligned}
$$

Note that $\left(\boldsymbol{\Sigma}_{11}^{(k-1)}-\boldsymbol{\Sigma}_{12}^{(k-1)} \cdot\left[\boldsymbol{\Sigma}_{22}^{(k-1)}\right]^{-1}\left[\boldsymbol{\Sigma}_{12}^{(k-1)}\right]^{\top}\right) \cdot \Delta_{k}^{2 H}$ is the conditional covariance matrix

$$
\mathbb{C o v}\left[\left(B^{H}\left(t_{1}^{k}\right), B^{H}\left(t_{3}^{k}\right), \cdots, B^{H}\left(t_{2^{k}-1}^{k}\right)\right) \mid \boldsymbol{B}_{k-1}^{H}\right] .
$$

Hence, the diagonal entries in second part in (42) are nonnegative. In the next, we compute the diagonal entries of the first part in (42). We use $\xi_{i, j}$ and $\eta_{i, j}$ to denote the $(i, j)$-th entry of $\boldsymbol{\Sigma}_{22}^{(k-1)}$ and $\boldsymbol{\Sigma}_{12}^{(k-1)}$, respectively. After some calculation, we obtain that the $j$-th diagonal entries of $\boldsymbol{M}_{k-1} \boldsymbol{\Sigma}_{22}^{(k-1)} \boldsymbol{M}_{k-1}^{\top}$ and $\boldsymbol{M}_{k-1}\left[\boldsymbol{\Sigma}_{12}^{(k-1)}\right]^{\top}$ are

$$
\begin{aligned}
{\left[\boldsymbol{M}_{k-1} \boldsymbol{\Sigma}_{22}^{(k-1)} \boldsymbol{M}_{k-1}^{\top}\right]_{j, j} } & =1 / 4 \cdot\left(\xi_{j, j}+\xi_{j, j+1}+\xi_{j+1, j}+\xi_{j+1, j+1}\right), \\
{\left[\boldsymbol{M}_{k-1}\left[\boldsymbol{\Sigma}_{12}^{(k-1)}\right]^{\top}\right]_{j, j} } & =1 / 2 \cdot\left(\eta_{j, j}+\eta_{j+1, j}\right) .
\end{aligned}
$$

By plugging in expression of $\xi_{i, j}, \eta_{i, j}$ and $\boldsymbol{\Sigma}_{11}^{(k-1)}$, we obtain that the $j$-th diagonal entry of the first part in (42) is $1-2^{2 H-2}$, which is a constant. As a result, the $j$-th diagonal entry of $\boldsymbol{\Sigma}^{(k)}$ is upper bounded by

$$
\left[\boldsymbol{\Sigma}^{(k)}\right]_{j, j} \leq\left(1-2^{2 H-2}\right) \cdot \Delta_{k}^{2 H}<2 \cdot 2^{-2 k H}
$$

A.2. Proof of Theorem 2 We first prove that the sequence $\left\{B_{n}^{H}\right\}_{n \geq 1}$ is a Cauchy sequence in $\mathcal{C}([0,1])$ almost surely. Then its limiting process exists almost surely due to the completeness of $\mathcal{C}([0,1])$. Second, we show that the limiting process is a Gaussian process and has the same covariance structure as the fBM. As a result, the almost surely limit of $B_{n}^{H}$ is fBM.

Since the tail bound $2 \exp \left\{-\nu^{* 2} \cdot 2^{2 k \delta-2}\right\}$ established in Theorem 1 is summable, by Borel-Cantelli Lemma, we have

$$
\mathbb{P}\left(\max _{0 \leq j \leq 2^{k-1}-1}\left|a_{j}^{k}-b_{j}^{k}\right| \geq \rho \ell_{k}, \text { i.o. }\right)=0 .
$$

Hence, there exists a random variable $N$, which is finite almost surely, such that for all $k \geq N$, $\max _{0 \leq j \leq 2^{k-1}-1}\left|a_{j}^{k}-b_{j}^{k}\right| \leq \rho \ell_{k}$. Then for arbitrary $\epsilon>0$, when $n, m$ large enough, we have

$$
\left\|B_{n}^{H}-B_{m}^{H}\right\|_{\infty} \leq \sum_{k=n+1}^{m}\left\|B_{k}^{H}-B_{k-1}^{H}\right\|_{\infty} \leq \sum_{k=n+1}^{m} \max _{0 \leq j \leq 2^{k-1}-1}\left|a_{j}^{k}-b_{j}^{k}\right| \leq \rho \sum_{k=n+1}^{m} \ell_{k}<\epsilon .
$$


Thus, by definition, $\left\{B_{n}^{H}\right\}_{n \geq 1}$ is a Cauchy sequence in $\mathcal{C}([0,1])$ almost surely. Since $\mathcal{C}([0,1])$ is complete, there exists a stochastic process $X$ such that $\left\|B_{n}^{H}-X\right\|_{\infty}$ converge to 0 almost surely.

We next show that $X$ is indeed a fBM. Consider an arbitrary finite collection of time points $\left(t_{1}^{*}, \ldots, t_{m}^{*}\right) \in[0,1]$. For each $1 \leq i \leq m$, there exists a sequence of points $t_{n}^{n_{i}} \in D_{n}$ such that $t_{n}^{n_{i}} \rightarrow t_{i}^{*}$. Note that

$$
\begin{aligned}
\left|X\left(t_{i}^{*}\right)-B_{n}^{H}\left(t_{n}^{n_{i}}\right)\right| & \leq\left|X\left(t_{i}^{*}\right)-X\left(t_{n}^{n_{i}}\right)\right|+\left|X\left(t_{n}^{n_{i}}\right)-B_{n}^{H}\left(t_{n}^{n_{i}}\right)\right| \\
& \leq\left|X\left(t_{i}^{*}\right)-X\left(t_{n}^{n_{i}}\right)\right|+\left\|X-B_{n}^{H}\right\|_{\infty}
\end{aligned}
$$

and $B_{n}^{H}\left(t_{n}^{i_{n}}\right)$ is centered and Gaussian. It implies that $\left(X\left(t_{1}^{*}\right), \cdots, X\left(t_{m}^{*}\right)\right)$ is the strong limit of a sequence of centered Gaussian random vectors.

In what follows, we show that the strong limit of a sequence of $m$-dimensional centered Gaussian random vector is still centered and Gaussian. Let $\left\{\boldsymbol{Z}_{n}\right\}_{n \geq 1}$ be a sequence of centered Gaussian random vectors with covariance matrix $\boldsymbol{\Sigma}_{n}$, and $\boldsymbol{Z}$ be the strong limit of $\left\{\boldsymbol{Z}_{n}\right\}_{n \geq 1}$. Convergence almost surely implies that $\left\{\boldsymbol{Z}_{n}\right\}_{n \geq 1}$ converge to $\boldsymbol{Z}$ in distribution and corresponding characteristic functions $\phi_{n}(\boldsymbol{\eta})=\exp \left\{-\boldsymbol{\eta}^{\top} \boldsymbol{\Sigma}_{n} \boldsymbol{\eta} / 2\right\}$ also converge for any fixed $\boldsymbol{\eta} \in \mathbb{R}^{m}$. By setting $\boldsymbol{\eta}=\boldsymbol{e}_{i}$ and $\boldsymbol{e}_{i}+\boldsymbol{e}_{j}, 1 \leq i, j \leq m$, where $\boldsymbol{e}_{i}$ is the $i$-th standard basis of $\mathbb{R}^{m}$, we obtain that each element of $\boldsymbol{\Sigma}_{n}$ converges. Hence, $\boldsymbol{\Sigma}_{n}$ converges to some matrix $\boldsymbol{\Sigma}$. As a result, the characteristic function of $\boldsymbol{Z}$ takes form $\exp \left\{-\boldsymbol{\eta}^{\top} \boldsymbol{\Sigma} \boldsymbol{\eta} / 2\right\}$, which implies that $\boldsymbol{Z}$ is a centered Gaussian random vector.

The above analysis indicates that $\left(X\left(t_{1}^{*}\right), \ldots, X\left(t_{m}^{*}\right)\right)$ is itself also a centered Gaussian random vector and hence, $X$ is a centered Gaussian process. Moreover, by construction, the covariance matrix of $\left(B_{n}^{H}\left(t_{n}^{n_{1}}\right), \cdots, B_{n}^{H}\left(t_{n}^{n_{m}}\right)\right)$ is $\boldsymbol{\Sigma}^{H}\left(t_{n}^{n_{1}}, \ldots, t_{n}^{n_{m}}\right)$, where $\boldsymbol{\Sigma}^{H}$ is the covariance matrix function of fBM. Since $\boldsymbol{\Sigma}^{H}$ is continuous, we have $\boldsymbol{\Sigma}^{H}\left(t_{n}^{n_{1}}, \ldots, t_{n}^{n_{m}}\right) \rightarrow \boldsymbol{\Sigma}^{H}\left(t_{1}^{*}, \ldots, t_{m}^{*}\right)$, which implies that $X$ has same covariance matrix function as fBM. Thus $X$ is a fBM.

A.3. Proof of Theorem 3 The moment generating function of $N$ can be written as

$$
\mathbb{E}[\exp \{\eta N\}]=\mathbb{E}\left[\int_{0}^{\infty} 1\{\exp \{\eta N\} \geq u\} \mathrm{d} u\right]=\int_{0}^{\infty} \mathbb{P}(N \geq \log (u) / \eta) \mathrm{d} u
$$

We have

$$
\begin{aligned}
\mathbb{P}(N \geq \log (u) / \eta) & \leq \sum_{k=\lfloor\log (u) / \eta\rfloor}^{\infty} \mathbb{P}(\text { record is broken at level } k) \\
& \left.\leq \sum_{k=[\log (u) / \eta]}^{\infty} 2 \exp \left\{-\left(\nu^{*}\right)^{2} \cdot 2^{2 k \delta-2}\right\} \text { (by Theorem } 1\right) \\
& \leq C \exp \left\{-\left(\nu^{*}\right)^{2} \cdot u^{2 \delta / \eta}\right\},
\end{aligned}
$$

where $C$ is a constant sufficiently large. Since $\exp \left\{-\left(\nu^{*}\right)^{2} \cdot u^{2 \delta / \eta}\right\}$ is integrable, $\mathbb{E}[\exp \{\eta N\}]$ is finite.

Now for $n>N$, according to the representation (15), we have

$$
\left\|B^{H}-B_{n}^{H}\right\|_{\infty} \leq \rho \cdot \sum_{k=n+1}^{\infty} 2^{-(H-\delta) k}=\frac{\rho \cdot 2^{-(H-\delta)(n+1)}}{1-2^{-(H-\delta)}} .
$$

A.4. Proof of Lemma 4 We use $f_{k}(t)$ to denote $B_{k}^{H}(t)-B_{k-1}^{H}(t)$ and then by definition,

$$
\left\|B_{k}^{H}-B_{k-1}^{H}\right\|_{\alpha}=\left\|f_{k}\right\|_{\alpha}=\sup _{0 \leq s<t \leq 1} \frac{\left|f_{k}(s)-f_{k}(t)\right|}{|s-t|^{\alpha}} .
$$


Recall that $f_{k}\left(t_{j}^{k-1}\right)=0, f_{k}\left(t_{2 j+1}^{k}\right)=a_{j}^{k}-b_{j}^{k}$ and $f_{k}(t)$ is linear over intervals $\left[t_{2 j}^{k}, t_{2 j+1}^{k}\right]$ and $\left[t_{2 j+1}^{k}, t_{2 j+2}^{k}\right]$, where $j=0, \ldots, 2^{k-1}-1$. Note that $t_{2 j}^{k}=t_{j}^{k-1}$. Let

$$
\kappa=2^{k} \cdot \max _{0 \leq j \leq 2^{k-1}-1}\left|a_{j}^{k}-b_{j}^{k}\right| .
$$

Then $\kappa$ is the maximal slope of all linear pieces of $f_{k}(t)$. We make a discussion based on the locations of $s$ and $t$.

case 1: $|s-t| \leq 2^{-(k-1)}$. If there exists some $j^{*}$ such that $s, t \in\left[t_{2 j^{*}}^{k}, t_{2 j^{*}+2}^{k}\right]$, since $\kappa$ is the maximal slope, it is easy to show that $\left|f_{k}(s)-f_{k}(t)\right| \leq \kappa \cdot|s-t|$. Otherwise, there exists some $j^{*}$ such that $t_{2 j^{*}-1}^{k} \leq s<t_{2 j^{*}}^{k}<t \leq t_{2 j^{*}+1}^{k}$. Then we have

$$
\begin{aligned}
\left|f_{k}(s)-f_{k}(t)\right| & =\left|f_{k}(s)-f_{k}\left(t_{2 j^{*}}^{k}\right)+f_{k}\left(t_{2 j^{*}}^{k}\right)-f_{k}(t)\right| \leq\left|f_{k}(s)-f_{k}\left(t_{2 j^{*}}^{k}\right)\right|+\left|f_{k}\left(t_{2 j^{*}}^{k}\right)-f_{k}(t)\right| \\
& \leq 2 \kappa \cdot\left(\left|s-t_{2 j^{*}}^{k}\right|+\left|t-t_{2 j^{*}}^{k}\right|\right)=2 \kappa \cdot|s-t| .
\end{aligned}
$$

Hence, by definition, we obtain

$$
\frac{\left|f_{k}(s)-f_{k}(t)\right|}{|s-t|^{\alpha}} \leq 2 \kappa \cdot|s-t|^{1-\alpha} \leq 2 \kappa \cdot 2^{-(1-\alpha) k} \leq 2^{\alpha(k-1)+2} \cdot \max _{0 \leq j \leq 2^{k-1}-1}\left|a_{j}^{k}-b_{j}^{k}\right| .
$$

case 2: $|s-t|>2^{-(k-1)}$. In this case, there exist some $i<j$ such that $s \in\left[t_{i}^{k}, t_{i+1}^{k}\right]$ and $t \in\left[t_{j}^{k}, t_{j+1}^{k}\right]$. Then we have

$$
\begin{aligned}
\left|f_{k}(s)-f_{k}(t)\right| & =\left|f_{k}(s)-f_{k}\left(t_{i+1}^{k}\right)+f_{k}\left(t_{j}^{k}\right)-f_{k}(t)\right| \leq\left|f_{k}(s)-f_{k}\left(t_{i+1}^{k}\right)\right|+\left|f_{k}\left(t_{j}^{k}\right)-f_{k}(t)\right| \\
& \leq 2 \kappa \cdot\left(\left|s-t_{i+1}^{k}\right|+\left|t-t_{j}^{k}\right|\right) \leq 2^{-k+2} \cdot \kappa .
\end{aligned}
$$

Moreover, we have

$$
\frac{\left|f_{k}(s)-f_{k}(t)\right|}{|s-t|^{\alpha}} \leq \frac{2^{-k+2} \cdot \kappa}{2^{-\alpha(k-1)}}=2^{\alpha(k-1)+2} \cdot \max _{0 \leq j \leq 2^{k-1}-1}\left|a_{j}^{k}-b_{j}^{k}\right| .
$$

Above all, we obtain $\left\|B_{k}^{H}-B_{k-1}^{H}\right\|_{\alpha} \leq 2^{\alpha(k-1)+2} \cdot \max _{0 \leq j \leq 2^{k-1}-1}\left|a_{j}^{k}-b_{j}^{k}\right|$, which concludes the proof of Lemma 4.

A.5. Proof of Theorem 4 By the definition of $N$, for all $k>N$, we have $\max _{0 \leq j \leq 2^{k-1}-1} \mid a_{j}^{k}-$ $b_{j}^{k} \mid \leq \rho 2^{-(H-\delta) k}$. Then according to Lemma 4, we have

$$
\left\|B_{k}^{H}-B_{k-1}^{H}\right\|_{\alpha} \leq 2^{\alpha(k-1)+2} \cdot \rho 2^{-(H-\delta) k}=\rho 2^{2-\alpha} \cdot 2^{-(H-\alpha-\delta) k} .
$$

Hence, for $n>N$

$$
\left\|B^{H}-B_{n}^{H}\right\|_{\alpha} \leq \sum_{k=n+1}^{\infty}\left\|B_{k}^{H}-B_{k-1}^{H}\right\|_{\alpha} \leq \sum_{k=n+1}^{\infty} \rho 2^{2-\alpha} \cdot 2^{-(H-\alpha-\delta) k}=\frac{\rho 2^{2-\alpha} \cdot 2^{-(H-\alpha-\delta)(n+1)}}{1-2^{-(H-\alpha-\delta)}} .
$$

\section{Appendix B: Detailed Proofs for Theorems and Lemmas in Section 4}

B.1. An Auxiliary Lemma In this subsection, we prove an auxiliary lemma, which establishes an upper bound on $\Xi_{n}^{(m, k)}$, i.e., the conditional moment generating function of $\boldsymbol{\beta}^{\top} \boldsymbol{\alpha}_{n}(m, k)$. This lemma is used in the proofs of Lemma 5 and 6.

LEMma 9. Under the BCE condition, for $m \geq 1,1 \leq k \leq 2^{n+m-1}$ and $\theta>0$,

$$
\Xi_{n}^{(m, k)}(\theta) \leq \exp \left\{1 / 2 \cdot\left(\rho \ell_{n+m} \cdot \theta+\theta^{2} \cdot 2^{-2(n+m) H}\right)\right\}
$$


Proof of Lemma 9. We denote by

$$
\bar{\mu}_{n}(m, k)=\mathbb{E}\left[\boldsymbol{\beta}^{\top} \boldsymbol{\alpha}_{n}(m, k) \mid \boldsymbol{B}_{n}^{H}\right], \sigma_{n}^{2}(m, k)=\mathbb{V}\left(\boldsymbol{\beta}^{\top} \boldsymbol{\alpha}_{n}(m, k) \mid \boldsymbol{B}_{n}^{H}\right) .
$$

The key of the proof is to establish proper upper bounds for $\bar{\mu}_{n}(m, k)$ and $\sigma_{n}^{2}(m, k)$. We will first show that

$$
\sigma_{n}^{2}(m, k) \leq 2^{-2(n+m) H}
$$

Similar to the proof of Lemma 1, for Gaussian random variables, taking condition does not increase the variance. Hence, we have

$$
\begin{aligned}
& \mathbb{V}\left(\boldsymbol{\beta}^{\top} \boldsymbol{\alpha}_{n}(m, k) \mid \boldsymbol{B}_{n}^{H}\right) \leq \mathbb{V}\left(1 / 2 \cdot\left(B^{H}\left(t_{2 k-2}^{n+m}\right)+B^{H}\left(t_{2 k}^{n+m}\right)\right)-B^{H}\left(t_{2 k-1}^{n+m}\right)\right) \\
& \leq 2 \cdot \mathbb{V}\left(1 / 2 \cdot\left(B^{H}\left(t_{2 k-2}^{n+m}\right)-B^{H}\left(t_{2 k-1}^{n+m}\right)\right)+2 \cdot \mathbb{V}\left(1 / 2 \cdot\left(B^{H}\left(t_{2 k}^{n+m}\right)-B^{H}\left(t_{2 k-1}^{n+m}\right)\right)\right.\right.
\end{aligned}
$$

Since the fBM is a stationary process, we have

$$
\mathbb{V}\left(B^{H}\left(t_{2 k-1}^{n+m}\right)-B^{H}\left(t_{2 k-2}^{n+m}\right)\right)=\mathbb{V}\left(B^{H}\left(t_{2 k}^{n+m}\right)-B^{H}\left(\left(t_{2 k-1}^{n+m}\right)=\mathbb{V}\left(B^{H}\left(\Delta_{n+m}\right)\right)=\Delta_{n+m}^{2 H},\right.\right.
$$

which further implies that

$$
\sigma_{n}^{2}(m, k) \leq 2^{-2(n+m) H}
$$

Under the BCE condition, we have $\bar{\mu}_{n}(m, k) \leq \rho / 2 \cdot \ell_{n+m}$. Then the upper bound of the moment generating function $\Xi_{n}^{(m, k)}(\theta)$ holds.

B.2. Proof of Lemma 5 We prove the case when $\pi=+1$ only. The case when $\pi=-1$ follows analogously. To avoid confusion, we write $\Theta_{n}^{\pi}(m, k)$ and $\theta_{n}^{\pi}(m)$ when $\pi=+1$ as $\Theta_{n}^{+}(m, k)$ and $\theta_{n}^{+}(m)$ respectively.

Note that if there is a up-crossing record-breaker at level $n+m$ position $k$, then $\boldsymbol{\beta}^{\top} \boldsymbol{\alpha}_{n}(m, k)>$ $\rho \ell_{n+m}$. In this case, we have

$$
\begin{aligned}
\Theta_{n}^{+}(m, k) & =g_{n}(m)^{-1} \cdot 2^{n+m} \cdot \exp \left\{-\theta_{n}^{+}(m) \cdot \boldsymbol{\beta}^{\top} \boldsymbol{\alpha}_{n}(m, k)+\log \left(\Xi_{n}^{(m, k)}\left(\theta_{n}^{+}(m)\right)\right)\right\} \\
& \leq g_{n}(m)^{-1} \cdot 2^{n+m} \cdot \exp \left\{-\theta_{n}^{+}(m) \cdot \rho \ell_{n+m}\right\} \cdot \exp \left\{1 / 2 \cdot\left(\rho \theta_{n}^{+}(m) \ell_{n+m}+\theta_{n}^{+}(m)^{2} \cdot 2^{-2(n+m) H}\right)\right\} \\
& \leq g_{n}(m)^{-1} \cdot 2^{n+m} \cdot \exp \left\{-\rho / 2 \cdot \theta_{n}^{+}(m) \cdot 2^{-(n+m)(H-\delta)}+1 / 2 \cdot \theta_{n}^{+}(m)^{2} \cdot 2^{-2(n+m) H}\right\},
\end{aligned}
$$

where the second inequality follows from Lemma 9. Hence, by our choice of

$$
\theta_{n}^{+}(m)=\rho / 2 \cdot 2^{(n+m)(H+\delta)},
$$

we obtain

$$
\Theta_{n}^{+}(m, k) \leq g_{n}(m)^{-1} \cdot 2^{n+m} \cdot \exp \left\{-\rho^{2} / 8 \cdot 2^{2(n+m) \delta}\right\}=Z_{n} \leq 1
$$

for all $n>N^{*}(\rho, \delta)$. 
B.3. Proof of Lemma 6 We first consider the conditional expectation. Note that

$$
\bar{\mu}_{n}(m, k)=\mathbb{E}\left[\boldsymbol{\beta}^{\top} \boldsymbol{\alpha}_{n}(m, k) \mid \boldsymbol{B}_{n}^{H}\right]=(1 / 2,-1,1 / 2)^{\top} \boldsymbol{\Sigma}_{n}^{(m, k)} \boldsymbol{\Sigma}_{n}^{-1} \boldsymbol{B}_{n}^{H} .
$$

Since $\bar{\mu}_{n}(m, k)$ is the linear combination of Gaussian random variables, itself is also Gaussian. It is easy to see that $\mathbb{E}\left[\bar{\mu}_{n}(m, k)\right]=0$. For the variance, according to the decomposition of conditional variance, we have

$$
\mathbb{V}\left(\boldsymbol{\beta}^{\top} \boldsymbol{\alpha}_{n}(m, k)\right)=\mathbb{E}\left[\mathbb{V}\left(\boldsymbol{\beta}^{\top} \boldsymbol{\alpha}_{n}(m, k) \mid \boldsymbol{B}_{n}^{H}\right)\right]+\mathbb{V}\left(\mathbb{E}\left[\boldsymbol{\beta}^{\top} \boldsymbol{\alpha}_{n}(m, k) \mid \boldsymbol{B}_{n}^{H}\right]\right) .
$$

Thus we obtain

$$
\left.\mathbb{V}\left(\bar{\mu}_{n}(m, k)\right)=\mathbb{V}\left(\boldsymbol{\beta}^{\top} \boldsymbol{\alpha}_{n}(m, k)\right)\right)-\mathbb{E}\left[\mathbb{V}\left(\boldsymbol{\beta}^{\top} \boldsymbol{\alpha}_{n}(m, k) \mid \boldsymbol{B}_{n}^{H}\right)\right] \leq 2 \cdot 2^{-2(n+m) H},
$$

where the inequality follows from the proof of Lemma 9. For fixed $n, m, k$, we define the event

$$
\mathcal{E}_{n}(m, k)=\left\{\left|\bar{\mu}_{n}(m, k)\right|>\rho / 2 \cdot 2^{-(n+m)(H-\delta)}\right\} .
$$

Then we have

$$
\mathbb{P}\left(\mathcal{E}_{n}(m, k)\right) \leq C \exp \left\{-\rho^{2} / 8 \cdot 2^{2(n+m) \delta}\right\},
$$

where $C$ is some constant. Note that $\mathcal{E}_{n} \subseteq \cup_{m=1}^{\infty} \cup_{k=1}^{2^{n+m-1}} \mathcal{E}_{n}(m, k)$. Then we have

$$
\begin{aligned}
\mathbb{P}\left(\mathcal{E}_{n}\right) & \leq \sum_{m=1}^{\infty} 2^{n+m-1} \cdot \mathbb{P}\left(\mathcal{E}_{n}(m, k)\right) \\
& \leq C \cdot \sum_{m=1}^{\infty} 2^{n+m-1} \cdot \exp \left\{-\rho^{2} / 8 \cdot 2^{2(n+m) \delta}\right\} \leq C^{\prime} \cdot \exp \left\{-C^{\prime \prime} \cdot 2^{2 n \delta}\right\},
\end{aligned}
$$

where $C^{\prime}$ and $C^{\prime \prime}$ are some constants.

Then similar to the proof of Theorem 3, we obtain that the moment generating function of $\mathcal{N}_{\mathcal{E}}$, the last level where the BCE condition is violated, is finite everywhere.

B.4. Proof of Lemma 7 Recall that we have

$$
\bar{\mu}_{n}(m, k)=\mathbb{E}\left[\boldsymbol{\beta}^{\top} \boldsymbol{\alpha}_{n}(m, k) \mid \boldsymbol{B}_{n}^{H}\right]=(1 / 2,-1,1 / 2)^{\top} \boldsymbol{\Sigma}_{n}^{(m, k)} \boldsymbol{\Sigma}_{n}^{-1} \boldsymbol{B}_{n}^{H} .
$$

Here $\boldsymbol{\Sigma}_{n}$ is the covariance matrix of $\boldsymbol{B}_{n}^{H}$ and $\boldsymbol{\Sigma}_{n}^{(m, k)}$ is the covariance matrix of $\boldsymbol{\alpha}_{n}(m, k)$ and $\boldsymbol{B}_{n}^{H}$, which has form

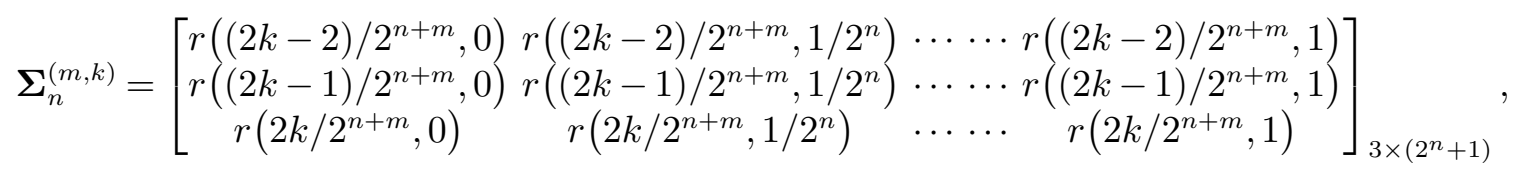

where $r(\cdot, \cdot)$ denotes the covariance function of fBM. By calculation, for $1 \leq j \leq 2^{n}+1$, the $j$-th entry of $(1 / 2,-1,1 / 2)^{\top} \boldsymbol{\Sigma}_{n}^{(m, k)}$ is

$$
\begin{aligned}
\frac{\Delta_{m+n}^{2 H}}{4} & \cdot\left(|2 k-2|^{2 H}+|2 k|^{2 H}-2|2 k-1|^{2 H}\right. \\
& \left.+\left|2 k-2-j 2^{m}\right|^{2 H}+\left|2 k-j 2^{m}\right|^{2 H}-2\left|2 k-1-j 2^{m}\right|^{2 H}\right) .
\end{aligned}
$$

We consider the function $\phi(x)=(x+2)^{2 H}+x^{2 H}-2 \cdot(x+1)^{2 H}$. Then we have $\phi^{\prime}(x)=2 H \cdot((x+$ $\left.2)^{2 H-1}+x^{2 H-1}-2 \cdot(x+1)^{2 H-1}\right)$. When $1 / 2<H<1$, by Jensen's inequality, $\phi^{\prime}(x) \leq 0$ for $x \geq 0$. 
Hence $\phi(x)$ is decreasing on $[0, \infty]$. Moreover, $\phi(x)$ is convex on $[0, \infty]$. As a result, for all positive integer $i$, we have

$$
0 \leq(i+2)^{2 H}+i^{2 H}-2 \cdot(i+1)^{2 H} \leq 2^{2 H}-2<2 .
$$

When $0<H<1 / 2$, by Jensen's inequality, $\phi^{\prime}(x) \geq 0$ for $x \geq 0$. Hence $\phi(x)$ is increasing on $[0, \infty]$. Moreover, $\phi(x)$ is concave on $[0, \infty]$. As a result, for all positive integer $i$, we have

$$
-2<2^{2 H}-2 \leq(i+2)^{2 H}+i^{2 H}-2 \cdot(i+1)^{2 H} \leq 0 .
$$

Hence, for all $H$ and $i$, we have || $2 i-\left.2\right|^{2 H}+|2 i|^{2 H}-2|2 i-1|^{2 H} \mid \leq 2$, which implies that the absolute value of any entry of $(1 / 2,-1,1 / 2)^{\top} \boldsymbol{\Sigma}_{n}^{(m, k)}$ is bounded by $\Delta_{n+m}^{2 H}=2^{-2(n+m) H}$. Recall that $\gamma_{n}$ denotes the maximal absolute value of the entries in vector $\boldsymbol{\Sigma}_{n}^{-1} \boldsymbol{B}_{n}^{H}$. Hence, we have

$$
\left|\bar{\mu}_{n}(m, k)\right|=\left|(1 / 2,-1,1 / 2)^{\top} \boldsymbol{\Sigma}_{n}^{(m, k)} \boldsymbol{\Sigma}_{n}^{-1} \boldsymbol{B}_{n}^{H}\right| \leq \gamma_{n} \cdot\left(2^{n}+1\right) \cdot 2^{-2(n+m) H} .
$$

\section{Appendix C: Convergence of the Euler scheme}

C.1. Auxiliary lemmas The proof of Theorem 9 relies on the several lemmas. The first one establishes the existence of the solution to equation (37).

Lemma 10. Under Assumption 1, the solution of equation (37) $\boldsymbol{y}$ exists. We also have the following estimates. For all $0 \leq s<t \leq 1$,

$$
\begin{aligned}
& \|\boldsymbol{y}(t)-\boldsymbol{y}(s)\| \leq G_{1}^{*} \cdot|t-s|^{\alpha} \\
& \|\boldsymbol{y}(t)-\boldsymbol{y}(s)-\boldsymbol{f}(\boldsymbol{y}(s)) \cdot(\boldsymbol{x}(t)-\boldsymbol{x}(s))\| \leq G_{2}^{*} \cdot|s-t|^{2 \alpha} .
\end{aligned}
$$

where $G_{1}^{*}$ and $G_{2}^{*}$ are defined in equation (40).

The next lemma is a counterpart of Lemma 10. It establishes similar properties for the solutions obtain by the Euler scheme.

Lemma 11. For all the dyadic discretization time points $t_{j}^{n}, t_{r}^{n} \in[0,1]$, we have the estimates

$$
\begin{aligned}
& \left\|\boldsymbol{y}_{n}\left(t_{j}^{n}\right)-\boldsymbol{y}_{n}\left(t_{r}^{n}\right)\right\| \leq G_{1} \cdot\left|t_{j}^{n}-t_{r}^{n}\right|^{\alpha} \\
& \left\|\boldsymbol{y}_{n}\left(t_{j}^{n}\right)-\boldsymbol{y}_{n}\left(t_{r}^{n}\right)-\boldsymbol{f}\left(\boldsymbol{y}_{n}\left(t_{r}^{n}\right)\right) \cdot\left(\boldsymbol{x}\left(t_{j}^{n}\right)-\boldsymbol{x}\left(t_{r}^{n}\right)\right)\right\| \leq G_{2} \cdot\left|t_{j}^{n}-t_{r}^{n}\right|^{2 \alpha} .
\end{aligned}
$$

where $G_{1}$ and $G_{2}$ are defined in equation (40).

We also need to define a restricted $\alpha$-Hölder norm. Specifically, given the dyadic partition $D_{n}$ and a path $\boldsymbol{x}(t)$, the restricted $\alpha$-Hölder norm on $D_{n}$ is defined as

$$
H_{\alpha}\left(\boldsymbol{x} \mid D_{n}\right)=\sup _{0 \leq i<j \leq 2^{n}} \frac{\left\|\boldsymbol{x}\left(t_{i}^{n}\right)-\boldsymbol{x}\left(t_{j}^{n}\right)\right\|}{\left|t_{i}^{n}-t_{j}^{n}\right|^{\alpha}} .
$$

Note that in the restricted $\alpha$-Hölder norm, we do not require that the path is well-defined on points outside of $D_{n}$. Intuitively, the restricted $\alpha$-Hölder norm measures the $\alpha$-Hölder continuity of the solution obtained via Euler scheme on $D_{n}$. We have the following lemma on the restricted $\alpha$-Hölder norm of $\boldsymbol{f}(\boldsymbol{y})-\boldsymbol{f}\left(\boldsymbol{y}_{n}\right)$ on $D_{n}$.

Lemma 12. For dyadic discretization $D_{n}$,

$$
\begin{gathered}
H_{\alpha}\left(\boldsymbol{f}(\boldsymbol{y})-\boldsymbol{f}\left(\boldsymbol{y}_{n}\right) \mid D_{n}\right) \leq\left(d \cdot\|\nabla \boldsymbol{f}\|+d^{2} \cdot\left\|\nabla^{2} \boldsymbol{f}\right\| \cdot\left(G_{1}^{*}+G_{1}\right)\right) \cdot H_{\alpha}\left(\boldsymbol{y}-\boldsymbol{y}_{n} \mid D_{n}\right)+ \\
d^{2} \cdot\left\|\nabla^{2} \boldsymbol{f}\right\| \cdot\left(G_{1}^{*}+G_{1}\right) \cdot\left\|\boldsymbol{y}(0)-\boldsymbol{y}_{n}(0)\right\| .
\end{gathered}
$$




\section{C.2. Proof of Theorem 9. Let}

$$
\mathcal{J}_{i} \equiv \int_{t_{i}^{n}}^{t_{i+1}^{n}}\left(\boldsymbol{f}(\boldsymbol{y}(s))-\boldsymbol{f}\left(\boldsymbol{y}\left(t_{i}^{n}\right)\right)\right) \mathrm{d} \boldsymbol{x}(s)
$$

By definition of Euler scheme and the solution of equation 37, we have

$$
\boldsymbol{y}_{n}\left(t_{i+1}^{n}\right)-\boldsymbol{y}\left(t_{i+1}^{n}\right)=\boldsymbol{y}_{n}\left(t_{i}^{n}\right)-\boldsymbol{y}\left(t_{i}^{n}\right)+\left(\boldsymbol{f}\left(\boldsymbol{y}_{n}\left(t_{i}^{n}\right)\right)-\boldsymbol{f}\left(\boldsymbol{y}\left(t_{i}^{n}\right)\right)\right) \cdot\left(\boldsymbol{x}\left(t_{i+1}^{n}\right)-\boldsymbol{x}\left(t_{i}^{n}\right)\right)-\mathcal{J}_{i},
$$

and furthermore, for all $0 \leq \ell<k \leq 2^{n}$,

$$
\left(\boldsymbol{y}_{n}\left(t_{k}^{n}\right)-\boldsymbol{y}\left(t_{k}^{n}\right)\right)-\left(\boldsymbol{y}_{n}\left(t_{\ell}^{n}\right)-\boldsymbol{y}\left(t_{\ell}^{n}\right)\right)=\sum_{i=\ell}^{k-1}\left(\boldsymbol{f}\left(\boldsymbol{y}_{n}\left(t_{i}^{n}\right)\right)-\boldsymbol{f}\left(\boldsymbol{y}\left(t_{i}^{n}\right)\right)\right) \cdot\left(\boldsymbol{x}\left(t_{i+1}^{n}\right)-\boldsymbol{x}\left(t_{i}^{n}\right)\right)-\sum_{i=\ell}^{k-1} \mathcal{J}_{i} .
$$

Using the Young-Lóeve estimate, we have the following bounds

$$
\begin{gathered}
\left\|\sum_{i=\ell}^{k-1}\left(\boldsymbol{f}\left(\boldsymbol{y}_{n}\left(t_{i}^{n}\right)\right)-\boldsymbol{f}\left(\boldsymbol{y}\left(t_{i}^{n}\right)\right)\right) \cdot\left(\boldsymbol{x}\left(t_{i+1}^{n}\right)-\boldsymbol{x}\left(t_{i}^{n}\right)\right)-\left(\boldsymbol{f}\left(\boldsymbol{y}_{n}\left(t_{\ell}^{n}\right)\right)-\boldsymbol{f}\left(\boldsymbol{y}\left(t_{\ell}^{n}\right)\right)\right) \cdot\left(\boldsymbol{x}\left(t_{k}^{n}\right)-\boldsymbol{x}\left(t_{\ell}^{n}\right)\right)\right\| \\
\leq h \cdot K(2 \alpha) \cdot H_{\alpha}\left(\boldsymbol{f}(\boldsymbol{y})-\boldsymbol{f}\left(\boldsymbol{y}_{n}\right) \mid D_{n}\right) \cdot C_{\alpha} \cdot\left|t_{k}^{n}-t_{\ell}^{n}\right|^{2 \alpha}
\end{gathered}
$$

where $K(2 \alpha)=1+\sum_{n=1}^{\infty} n^{-2 \alpha}$ and $C_{\alpha}$ is the $\alpha$-Hölder norm of $\boldsymbol{x}$. By Lemma 10, we also have

$$
\left\|\mathcal{J}_{i}\right\| \leq G_{2}^{*} \cdot\left|t_{i+1}^{n}-t_{i}^{n}\right|^{2 \alpha} .
$$

Note that

$$
\sum_{i=\ell}^{k-1}\left|t_{i+1}^{n}-t_{i}^{n}\right|^{2 \alpha} \leq\left|t_{k}^{n}-t_{\ell}^{n}\right| \cdot \Delta_{n}^{2 \alpha-1} \leq\left|t_{k}^{n}-t_{\ell}^{n}\right|^{\alpha} \cdot \Delta_{n}^{2 \alpha-1}
$$

Then we have

$$
\begin{aligned}
\|\left(\boldsymbol{y}_{n}\left(t_{k}^{n}\right)-\right. & \left.\boldsymbol{y}\left(t_{k}^{n}\right)\right)-\left(\boldsymbol{y}_{n}\left(t_{\ell}^{n}\right)-\boldsymbol{y}\left(t_{\ell}^{n}\right)\right) \| \\
& \leq h \cdot K(2 \alpha) \cdot H_{\alpha}\left(\boldsymbol{f}(\boldsymbol{y})-\boldsymbol{f}\left(\boldsymbol{y}_{n}\right) \mid D_{n}\right) \cdot C_{\alpha} \cdot\left|t_{k}^{n}-t_{\ell}^{n}\right|^{2 \alpha}+ \\
& \left(\boldsymbol{f}\left(\boldsymbol{y}_{n}\left(t_{\ell}^{n}\right)\right)-\boldsymbol{f}\left(\boldsymbol{y}\left(t_{\ell}^{n}\right)\right)\right) \cdot\left(\boldsymbol{x}\left(t_{k}^{n}\right)-\boldsymbol{x}\left(t_{\ell}^{n}\right)\right)+G_{2}^{*} \cdot\left|t_{k}^{n}-t_{\ell}^{n}\right|^{\alpha} \cdot \Delta_{n}^{2 \alpha-1} .
\end{aligned}
$$

By the definition of restricted $\alpha$-Hölder norm,

$$
\left\|\boldsymbol{f}\left(\boldsymbol{y}_{n}\left(t_{\ell}^{n}\right)\right)-\boldsymbol{f}\left(\boldsymbol{y}\left(t_{\ell}^{n}\right)\right)\right\| \leq H_{\alpha}\left(\boldsymbol{f}(\boldsymbol{y})-\boldsymbol{f}\left(\boldsymbol{y}_{n}\right) \mid D_{n}\right) \cdot\left|t_{\ell}^{n}\right|^{\alpha}+\left\|\boldsymbol{f}\left(\boldsymbol{y}_{n}(0)\right)-\boldsymbol{f}(\boldsymbol{y}(0))\right\|,
$$

combined with Lemma 12, we have

$$
\begin{aligned}
& H_{\alpha}\left(\boldsymbol{y}-\boldsymbol{y}_{n} \mid D_{n}\right) \leq h \cdot K(2 \alpha) \cdot\left(\left|t_{k}^{n}-t_{\ell}^{n}\right|^{\alpha}+\left|t_{\ell}^{n}\right|^{\alpha}\right) \cdot C_{\alpha} \cdot H_{\alpha}\left(\boldsymbol{f}(\boldsymbol{y})-\boldsymbol{f}\left(\boldsymbol{y}_{n}\right) \mid D_{n}\right)+ \\
& \qquad G_{2}^{*} \cdot \Delta_{n}^{2 \alpha-1}+C_{\alpha} \cdot\left\|\boldsymbol{f}\left(\boldsymbol{y}_{n}(0)\right)-\boldsymbol{f}(\boldsymbol{y}(0))\right\| \\
& \leq h \cdot K(2 \alpha) \cdot C_{\alpha} \cdot\left(d \cdot\|\nabla \boldsymbol{f}\|+d^{2} \cdot\left\|\nabla^{2} \boldsymbol{f}\right\| \cdot\left(G_{1}^{*}+G_{1}\right)\right) \cdot\left(\left|t_{k}^{n}-t_{\ell}^{n}\right|^{\alpha}+\left|t_{\ell}^{n}\right|^{\alpha}\right) \\
& \times H_{\alpha}\left(\boldsymbol{y}-\boldsymbol{y}_{n} \mid D_{n}\right)+G_{2}^{*} \cdot \Delta_{n}^{2 \alpha-1}+C_{\alpha} \cdot\left(d^{2} h \cdot K(2 \alpha) \cdot\left\|\nabla^{2} \boldsymbol{f}\right\| \cdot\left(G_{1}^{*}+G_{1}\right)+d \cdot\|\nabla \boldsymbol{f}\|\right) \\
& \times\left\|\boldsymbol{y}_{n}(0)-\boldsymbol{y}(0)\right\| .
\end{aligned}
$$

By the definition of $\zeta$ and $v$ in (41),

$$
H_{\alpha}\left(\boldsymbol{y}-\boldsymbol{y}_{n} \mid D_{n}\right) \leq \zeta\left(\left|t_{k}^{n}-t_{\ell}^{n}\right|^{\alpha}+\left|t_{\ell}^{n}\right|^{\alpha}\right) \cdot H_{\alpha}\left(\boldsymbol{y}-\boldsymbol{y}_{n} \mid D_{n}\right)+G_{2}^{*} \cdot \Delta_{n}^{2 \alpha-1}+v\left\|\boldsymbol{y}_{n}(0)-\boldsymbol{y}(0)\right\| .
$$


Let $T_{0}=0$ and $T_{1}=\max \left\{t \in D_{n}, t \leq(4 \zeta)^{-1 / \alpha}\right\}$. Then

$$
H_{\alpha}\left(\boldsymbol{y}-\boldsymbol{y}_{n} \mid D_{n} \cap\left[T_{0}, T_{1}\right]\right) \leq 2 G_{2}^{*} \cdot \Delta_{n}^{2 \alpha-1}+2 v\left\|\boldsymbol{y}_{n}(0)-\boldsymbol{y}(0)\right\|
$$

Since the Euler scheme and the exact solution have same initial value, $\left\|\boldsymbol{y}_{n}(0)-\boldsymbol{y}(0)\right\|=0$, then we have

$$
H_{\alpha}\left(\boldsymbol{y}-\boldsymbol{y}_{n} \mid D_{n} \cap\left[T_{0}, T_{1}\right]\right) \leq 2 G_{2}^{*} \cdot \Delta_{n}^{2 \alpha-1},
$$

and furthermore,

$$
\left\|\boldsymbol{y}_{n}\left(T_{1}\right)-\boldsymbol{y}\left(T_{1}\right)\right\| \leq 2 G_{2}^{*} \cdot(4 \zeta)^{-1} \cdot \Delta_{n}^{2 \alpha-1}
$$

Now we let $T_{2}=\max \left\{t \in D_{n}, t \leq 2(4 \zeta)^{-1 / \alpha}\right\}$. For $T_{1} \leq t_{\ell}^{n}<t_{k}^{n} \leq T_{2}$, we have

$$
H_{\alpha}\left(\boldsymbol{y}-\boldsymbol{y}_{n} \mid D_{n}\right) \leq \zeta\left(\left|t_{k}^{n}-t_{\ell}^{n}\right|^{\alpha}+\left|t_{\ell}^{n}-T_{1}\right|^{\alpha}\right) \cdot H_{\alpha}\left(\boldsymbol{y}-\boldsymbol{y}_{n} \mid D_{n}\right)+G_{2}^{*} \cdot \Delta_{n}^{2 \alpha-1}+v\left\|\boldsymbol{y}_{n}\left(T_{1}\right)-\boldsymbol{y}\left(T_{2}\right)\right\| .
$$

As a result,

$$
H_{\alpha}\left(\boldsymbol{y}-\boldsymbol{y}_{n} \mid D_{n} \cap\left[T_{1}, T_{2}\right]\right) \leq 2\left(G_{2}^{*}+v \cdot 2 G_{2}^{*} \cdot(4 \zeta)^{-1}\right) \cdot \Delta_{n}^{2 \alpha-1},
$$

and

$$
\left\|\boldsymbol{y}_{n}\left(T_{2}\right)-\boldsymbol{y}\left(T_{2}\right)\right\| \leq\left[2\left(G_{2}^{*}+v \cdot 2 G_{2}^{*} \cdot(4 \zeta)^{-1}\right) \cdot(4 \zeta)^{-1}+2 G_{2}^{*} \cdot(4 \zeta)^{-1}\right] \cdot \Delta_{n}^{2 \alpha-1}
$$

We need to repeat this procedure at most $k^{*}=\left\lceil(4 \zeta)^{1 / \alpha}\right\rceil$ times in order to cover the whole interval $[0,1]$ and we can obtain the a sequence of bounds

$$
\begin{aligned}
H_{\alpha}\left(\boldsymbol{y}-\boldsymbol{y}_{n} \mid D_{n} \cap\left[T_{k-1}, T_{k}\right]\right) & \leq \Gamma_{k} \cdot \Delta_{n}^{2 \alpha-1}, \\
\left\|\boldsymbol{y}_{n}\left(T_{k}\right)-\boldsymbol{y}\left(T_{k}\right)\right\| & \leq \Upsilon_{k} \cdot \Delta_{n}^{2 \alpha-1}
\end{aligned}
$$

where $\left\{\Gamma_{k}\right\}$ and $\left\{\Upsilon_{k}\right\}$ are defined via recursion (41). Note that $\left\{\Gamma_{k}\right\}$ and $\left\{\Upsilon_{k}\right\}$ are increasing sequences, and for any $t_{i}^{n} \in\left[T_{k-1}, T_{k}\right]$, we have $\left\|\boldsymbol{y}_{n}\left(t_{i}^{n}\right)-\boldsymbol{y}\left(t_{i}^{n}\right)\right\| \leq \Upsilon_{k} \cdot \Delta_{n}^{2 \alpha-1}$. Therefore,

$$
\sup _{t_{i}^{n} \in D_{n}}\left\|\boldsymbol{y}_{n}\left(t_{i}^{n}\right)-\boldsymbol{y}\left(t_{i}^{n}\right)\right\| \leq \Upsilon_{\left\lceil(4 \zeta)^{1 / \alpha}\right\rceil} \cdot \Delta_{n}^{2 \alpha-1} .
$$

Finally, for any $t \in[0,1]$, there exists $i$ such that $t_{i}^{n} \leq t<t_{i+1}^{n}$. Then we have

$$
\begin{aligned}
\left\|\boldsymbol{y}_{n}(t)-\boldsymbol{y}(t)\right\| & \leq\left\|\boldsymbol{y}_{n}\left(t_{i}^{n}\right)-\boldsymbol{y}_{n}(t)\right\|+\left\|\boldsymbol{y}_{n}(t)-\boldsymbol{y}(t)\right\|+\left\|\boldsymbol{y}\left(t_{i}^{n}\right)-\boldsymbol{y}(t)\right\| \\
& \leq \Upsilon_{\left\lceil(4 \zeta)^{1 / \alpha}\right\rceil} \cdot \Delta_{n}^{2 \alpha-1}+G_{1}^{*} \cdot \Delta_{n}^{\alpha} \leq\left(\Upsilon_{\left\lceil(4 \zeta)^{1 / \alpha}\right\rceil}+G_{1}^{*}\right) \cdot \Delta_{n}^{2 \alpha-1} .
\end{aligned}
$$

\section{C.3. Proof of the auxiliary lemmas}

C.3.1. Proof of Lemma 10 Let $\omega=\left(2 d h C_{\alpha} K(2 \alpha)|\nabla \boldsymbol{f}|\right)^{-1 / \alpha}$ and $T_{k}=k \omega, k=0,1,2, \cdots$. Then the union of $\left[T_{k}, T_{k+1}\right]$ where $k=0, \cdots,\left\lceil\omega^{-1}\right\rceil$, covers $[0,1]$. From now on, we use $H_{\alpha,[s, t]}(\boldsymbol{u})$ to denote the $\alpha$-Hölder norm of $u$ on interval $[s, t]$. To be precise,

$$
H_{\alpha,[s, t]}(\boldsymbol{u})=\sup _{s \leq s^{\prime}<t^{\prime} \leq t} \frac{\left\|\boldsymbol{u}\left(s^{\prime}\right)-\boldsymbol{u}\left(t^{\prime}\right)\right\|}{\left|s^{\prime}-t^{\prime}\right|^{\alpha}} .
$$


We introduce this new notation because in our later proof, we need to consider the $\alpha$-Hölder norm of $\boldsymbol{u}$ defined on some subintervals of $[0,1]$. According to the property of Young integral, we have that for all $s, t \in\left[T_{k}, T_{k+1}\right]$,

$$
\begin{aligned}
\left\|\int_{s}^{t} \boldsymbol{f}(\boldsymbol{y}(u)) \mathrm{d} \boldsymbol{x}(u)\right\| & \leq\left|\boldsymbol{f}(\boldsymbol{y}(s))^{T}(\boldsymbol{x}(t)-\boldsymbol{x}(s))\right|+d \cdot K(2 \alpha) \cdot H_{\alpha,\left[T_{k}, T_{k+1}\right]}(\boldsymbol{f}(\boldsymbol{y})) \cdot C_{\alpha} \cdot|s-t|^{\alpha} \\
& \leq\left(h \cdot\|\boldsymbol{f}\| \cdot C_{\alpha}+d h \cdot K(2 \alpha) \cdot C_{\alpha} \cdot\|\nabla \boldsymbol{f}\| \cdot H_{\alpha,\left[T_{k}, T_{k+1}\right]}(\boldsymbol{y}) \cdot|s-t|^{\alpha}\right) \cdot|s-t|^{\alpha} .
\end{aligned}
$$

Since $\boldsymbol{y}$ is the solution of equation (37), it is easy to see that

$$
H_{\alpha,\left[T_{k}, T_{k+1}\right]}(\boldsymbol{y}) \leq h \cdot\|\boldsymbol{f}\| \cdot C_{\alpha}+d h \cdot K(2 \alpha) \cdot C_{\alpha} \cdot\|\nabla \boldsymbol{f}\| \cdot H_{\alpha,\left[T_{k}, T_{k+1}\right]}(\boldsymbol{y}) \cdot \omega^{\alpha},
$$

which further implies that

$$
\sup _{0 \leq k \leq\left\lfloor\omega^{-1}\right\rfloor} H_{\alpha,\left[T_{k}, T_{k+1}\right]}(\boldsymbol{y}) \leq 2 h \cdot\|\boldsymbol{f}\| \cdot C_{\alpha} .
$$

Now we turn to bound $H_{\alpha,[0,1]}(\boldsymbol{y})$. For any $s, t \in[0,1]$, we assume that $T_{i} \leq s<T_{i+1} \leq \cdots T_{j} \leq t<T_{j+1}$. Then we have

$$
\begin{aligned}
\|\boldsymbol{y}(s)-\boldsymbol{y}(t)\| & \leq\left\|\boldsymbol{y}(s)-\boldsymbol{y}\left(T_{i+1}\right)\right\|+\sum_{\ell=i+1}^{j-1}\left\|\boldsymbol{y}\left(T_{\ell}\right)-\boldsymbol{y}\left(T_{\ell+1}\right)\right\|+\left\|\boldsymbol{y}\left(T_{j}\right)-\boldsymbol{y}(t)\right\| \\
& \leq \sup _{0 \leq k \leq\left\lfloor\omega^{-1}\right\rfloor} H_{\alpha,\left[T_{k}, T_{k+1}\right]}(\boldsymbol{y}) \cdot\left(\left|s-T_{i+1}\right|^{\alpha}+\sum_{\ell=i+1}^{j-1}\left|T_{\ell}-T_{\ell+1}\right|^{\alpha}+\left|T_{j}-t\right|^{\alpha}\right) .
\end{aligned}
$$

Since $\alpha<1$, using Jensen's inequality and the number of covering subintervals, we have

$$
\left|s-T_{i+1}\right|^{\alpha}+\sum_{\ell=i+1}^{j-1}\left|T_{\ell}-T_{\ell+1}\right|^{\alpha}+\left|T_{j}-t\right|^{\alpha} \leq\left\lceil\omega^{-1}\right\rceil^{1-\alpha} \cdot|s-t|^{\alpha} .
$$

So we have $H_{\alpha,[0,1]}(\boldsymbol{y}) \leq 2 h \cdot\left\lceil\omega^{-1}\right\rceil^{1-\alpha} \cdot|\boldsymbol{f}| \cdot C_{\alpha}$, which is the first bound.

For another bound, note that according to Young-Lóeve estimate, for any partition $\Pi$ of $[s, t]$, we have

$$
\left\|\sum_{t_{i} \in \Pi} \boldsymbol{f}\left(\boldsymbol{y}\left(t_{i}\right)\right) \cdot\left(\boldsymbol{x}\left(t_{i+1}\right)-\boldsymbol{x}\left(t_{i}\right)\right)-\boldsymbol{f}(\boldsymbol{y}(s)) \cdot(\boldsymbol{x}(t)-\boldsymbol{x}(s))\right\| \leq d h \cdot K(2 \alpha) \cdot\|\nabla \boldsymbol{f}\| \cdot H_{\alpha,[0,1]}(\boldsymbol{y}) \cdot C_{\alpha}|s-t|^{2 \alpha} .
$$

Let the partition mesh goes to zero, we obtain the result.

C.3.2. Proof of Lemma 11 For notational convenience, in this proof, we use $\boldsymbol{x}_{k}$ to denote $\boldsymbol{x}\left(t_{k}^{n}\right)$ and $\boldsymbol{y}_{k}$ to denote $\boldsymbol{y}_{n}\left(t_{k}^{n}\right)$. We also write $y_{k}^{i}$ as the $i$-th element of $\boldsymbol{y}_{k}$. For each $0 \leq j \leq \ell \leq 2^{n}$, let $\boldsymbol{I}_{j \ell}=\boldsymbol{y}_{\ell}-\boldsymbol{y}_{j}-\boldsymbol{f}\left(\boldsymbol{y}_{j}\right) \cdot\left(\boldsymbol{x}_{j+1}-\boldsymbol{x}_{j}\right)$, and $I_{j \ell}^{i}$ denote the $i$-th element of $\boldsymbol{I}_{j \ell}$. We first show that for all $t_{r}^{n}, t_{j}^{n} \in[0,1]$, whenever $\left|t_{r}^{n}-t_{j}^{n}\right| \leq \omega$, then $\left\|\boldsymbol{I}_{j r}\right\| \leq L\left|t_{r}^{n}-t_{j}^{n}\right|^{2 \alpha}$. By the definition of Euler scheme, $\boldsymbol{I}_{j r}=0$, if $r-j=0,1$. For $r-j \geq 2$, we prove this lemma via induction. Suppose that the claim holds true for all pairs $p, q$ with $q-p<r-j$. Let $\ell \in[j, r)$ be the largest integer such that $\left|t_{j}^{n}-t_{\ell}^{n}\right| \leq 1 / 2 \cdot\left|t_{j}^{n}-t_{r}^{n}\right|$. Then we have $\left|t_{\ell+1}^{n}-t_{r}^{n}\right| \leq 1 / 2 \cdot\left|t_{j}^{n}-t_{r}^{n}\right|$. By the inductive hypothesis, $\left\|\boldsymbol{I}_{j \ell}\right\| \leq L\left|t_{\ell}^{n}-t_{j}^{n}\right|^{2 \alpha}$ and hence, we have

$$
\left|y_{\ell}^{i}-y_{j}^{i}\right| \leq\left|I_{j \ell}^{i}\right|+\left|\sum_{k=1}^{h} f_{i k}\left(\boldsymbol{y}_{j}\right)\left(x_{\ell}^{k}-x_{j}^{k}\right)\right| \leq L\left|t_{\ell}^{n}-t_{j}^{n}\right|^{2 \alpha}+h \cdot\|\boldsymbol{f}\| \cdot C_{\alpha}\left|t_{\ell}^{n}-t_{j}^{n}\right|^{\alpha} .
$$


Furthermore, since $\left|t_{\ell}^{n}-t_{j}^{n}\right| \leq \omega=\left(h|\boldsymbol{f}| C_{\alpha} / L\right)^{1 / \alpha}$, we have $\left\|\boldsymbol{y}_{\ell}-\boldsymbol{y}_{j}\right\| \leq 2 h \cdot\|\boldsymbol{f}\| \cdot C_{\alpha}\left|t_{\ell}-t_{j}\right|^{\alpha}$. Note that for $j \leq \ell \leq r$,

$$
I_{j r}^{i}=I_{j \ell}^{i}+I_{\ell r}^{i}+\sum_{k=1}^{h}\left(f_{i k}\left(\boldsymbol{y}_{\ell}\right)-f_{i k}\left(\boldsymbol{y}_{j}\right)\right)\left(x_{r}^{k}-x_{\ell}^{k}\right) .
$$

Then we have

$$
\begin{aligned}
\left|I_{j r}^{i}\right| & \leq\left|I_{j \ell}^{i}\right|+\left|I_{\ell r}^{i}\right|+h\|\nabla \boldsymbol{f}\| \cdot\left\|\boldsymbol{y}_{\ell}-\boldsymbol{y}_{j}\right\| \cdot C_{\alpha}\left|t_{r}^{n}-t_{\ell}^{n}\right|^{\alpha} \\
& \leq\left|I_{j \ell}^{i}\right|+\left|I_{\ell r}^{i}\right|+2 h^{2}\|\nabla \boldsymbol{f}\| \cdot\|\boldsymbol{f}\| \cdot C_{\alpha}^{2} \cdot\left|t_{r}^{n}-t_{j}^{n}\right|^{2 \alpha}
\end{aligned}
$$

Similarly, we have

$$
\left|I_{\ell r}^{i}\right| \leq\left|I_{\ell, \ell+1}^{i}\right|+\left|I_{\ell+1, r}^{i}\right|+2 h^{2}\|\nabla \boldsymbol{f}\| \cdot\|\boldsymbol{f}\| \cdot C_{\alpha}^{2} \cdot\left|t_{r}^{n}-t_{j}^{n}\right|^{2 \alpha} .
$$

Since $I_{\ell, \ell+1}^{i}=0$, we get

$$
\left|I_{j r}^{i}\right| \leq\left|I_{j \ell}^{i}\right|+\left|I_{\ell+1, r}^{i}\right|+\left(2 h C_{\alpha}\right)^{2}\|\nabla \boldsymbol{f}\| \cdot\|\boldsymbol{f}\| \cdot\left|t_{r}^{n}-t_{j}^{n}\right|^{2 \alpha} .
$$

By applying the inductive hypothesis again, we obtain

$$
\begin{aligned}
\left|I_{j r}^{i}\right| & \leq L\left(\left|t_{j}-t_{\ell}\right|^{2 \alpha}+\left|t_{\ell+1}-t_{r}\right|^{2 \alpha}\right)+\left(2 h C_{\alpha}\right)^{2}\|\nabla \boldsymbol{f}\| \cdot\|\boldsymbol{f}\| \cdot\left|t_{r}^{n}-t_{j}^{n}\right|^{2 \alpha} \\
& \leq\left(2^{1-2 \alpha} L+\left(2 h C_{\alpha}\right)^{2}\|\nabla \boldsymbol{f}\| \cdot\|\boldsymbol{f}\|\right) \cdot\left|t_{r}^{n}-t_{j}^{n}\right|^{2 \alpha} \\
& =L \cdot\left|t_{r}^{n}-t_{j}^{n}\right|^{2 \alpha}
\end{aligned}
$$

where $L=\left(1-2^{1-2 \alpha}\right)^{-1} \cdot\left(2 h C_{\alpha}\right)^{2}\|\nabla \boldsymbol{f}\| \cdot\|\boldsymbol{f}\|$. Hence, by induction, we finish the proof. Recall the definition of $\boldsymbol{I}_{j r}$, we also have that if $\left|t_{r}^{n}-t_{j}^{n}\right| \leq \omega$,

$$
\left\|\boldsymbol{y}_{r}-\boldsymbol{y}_{j}\right\| \leq\left\|\boldsymbol{I}_{j r}\right\|+h\|\boldsymbol{f}\| C_{\alpha}\left|t_{r}^{n}-t_{j}^{n}\right|^{\alpha} \leq\left(L+h\|\boldsymbol{f}\| C_{\alpha}\right) \cdot\left|t_{r}^{n}-t_{j}^{n}\right|^{\alpha} .
$$

Now we come back to the proof of Lemma 11. For $t_{r}^{n}, t_{j}^{n} \in[0,1]$ with $\left|t_{r}^{n}-t_{j}^{n}\right| \leq \omega$, we already obtain the conclusion. Otherwise, we can decompose the interval $\left[t_{r}^{n}, t_{j}^{n}\right]$ as

$$
t_{r}^{n}=t_{k_{0}}^{n}<t_{k_{1}}^{n}<t_{k_{2}}^{n}<\cdots<t_{k_{m}}^{n}=t_{j}^{n}
$$

such that $\left|t_{k_{i+1}}^{n}-t_{k_{i}}^{n}\right| \leq \omega$ or $k_{i+1}-k_{i}=1, i=0,1, \cdots m-1$. In either case, it is easy to see that

$$
\left\|\boldsymbol{y}_{k_{i+1}}-\boldsymbol{y}_{k_{i}}\right\| \leq\left(L+h\|\boldsymbol{f}\| C_{\alpha}\right) \cdot\left|t_{k_{i+1}}^{n}-t_{k_{i}}^{n}\right|^{\alpha}
$$

and hence

$$
\left\|\boldsymbol{y}_{j}-\boldsymbol{y}_{r}\right\| \leq\left(L+h\|\boldsymbol{f}\| C_{\alpha}\right) \cdot\left|t_{j}^{n}-t_{r}^{n}\right|^{\alpha} \cdot m \leq\left(L+h\|\boldsymbol{f}\| C_{\alpha}\right) \cdot\left(1+\omega^{-1}\right) \cdot\left|t_{j}^{n}-t_{r}^{n}\right|^{\alpha} .
$$

Furthermore, we have

$$
\begin{aligned}
\left\|\boldsymbol{I}_{j r}\right\| & \leq\left\|\boldsymbol{y}_{j}-\boldsymbol{y}_{r}\right\|+h\|\boldsymbol{f}\| C_{\alpha}\left|t_{j}^{n}-t_{r}^{n}\right|^{\alpha} \\
& \leq\left(2+\omega^{-1}\right) \cdot\left(L+h\|\boldsymbol{f}\| C_{\alpha}\right) \cdot\left|t_{j}^{n}-t_{r}^{n}\right|^{\alpha} \\
& \leq\left(2 \omega^{-\alpha}+\omega^{-1-\alpha}\right) \cdot\left(L+h\|\boldsymbol{f}\| C_{\alpha}\right) \cdot\left|t_{j}^{n}-t_{r}^{n}\right|^{2 \alpha}
\end{aligned}
$$

where we use $\left|t_{j}^{n}-t_{r}^{n}\right| \geq \omega$ in the last inequality. Hence we conclude the proof of Lemma 11. 
C.3.3. Proof of Lemma 12 Based on Taylor's expansion, for $t_{i}^{n}, t_{j}^{n} \in D_{n}$, we have

$$
\begin{aligned}
& \left\|\left[\boldsymbol{f}\left(\boldsymbol{y}\left(t_{i}^{n}\right)\right)-\boldsymbol{f}\left(\boldsymbol{y}\left(t_{j}^{n}\right)\right)\right]-\left[\boldsymbol{f}\left(\boldsymbol{y}_{n}\left(t_{i}^{n}\right)\right)-\boldsymbol{f}\left(\boldsymbol{y}_{n}\left(t_{j}^{n}\right)\right)\right]\right\| \\
= & \| \int_{0}^{1} \nabla \boldsymbol{f}\left(\boldsymbol{y}\left(t_{i}^{n}\right)+\tau \cdot\left[\boldsymbol{y}_{n}\left(t_{i}^{n}\right)-\boldsymbol{y}\left(t_{i}^{n}\right)\right]\right)^{\top}\left[\boldsymbol{y}\left(t_{i}^{n}\right)-\boldsymbol{y}_{n}\left(t_{i}^{n}\right)\right] \mathrm{d} \tau \\
& -\int_{0}^{1} \nabla \boldsymbol{f}\left(\boldsymbol{y}\left(t_{j}^{n}\right)+\tau \cdot\left[\boldsymbol{y}_{n}\left(t_{j}^{n}\right)-\boldsymbol{y}\left(t_{j}^{n}\right)\right]\right)^{\top}\left[\boldsymbol{y}\left(t_{j}^{n}\right)-\boldsymbol{y}_{n}\left(t_{j}^{n}\right)\right] \mathrm{d} \tau \| \\
\leq & \left\|\int_{0}^{1} \nabla \boldsymbol{f}\left(\boldsymbol{y}\left(t_{i}^{n}\right)+\tau \cdot\left[\boldsymbol{y}_{n}\left(t_{i}^{n}\right)-\boldsymbol{y}\left(t_{i}^{n}\right)\right]\right)^{\top}\left[\left(\boldsymbol{y}\left(t_{i}^{n}\right)-\boldsymbol{y}_{n}\left(t_{i}^{n}\right)\right)-\left(\boldsymbol{y}\left(t_{j}^{n}\right)-\boldsymbol{y}_{n}\left(t_{j}^{n}\right)\right)\right] \mathrm{d} \tau\right\| \\
& +\left\|\int_{0}^{1} \nabla \boldsymbol{f}\left(\boldsymbol{y}\left(t_{j}^{n}\right)+\tau \cdot\left[\boldsymbol{y}_{n}\left(t_{j}^{n}\right)-\boldsymbol{y}\left(t_{j}^{n}\right)\right]\right)-\nabla \boldsymbol{f}\left(\boldsymbol{y}\left(t_{i}^{n}\right)+\tau \cdot\left[\boldsymbol{y}_{n}\left(t_{i}^{n}\right)-\boldsymbol{y}\left(t_{i}^{n}\right)\right]\right)^{\top}\left[\boldsymbol{y}\left(t_{j}^{n}\right)-\boldsymbol{y}_{n}\left(t_{j}^{n}\right)\right] \mathrm{d} \tau\right\| .
\end{aligned}
$$

We use $A$ and $C$ to denote the two parts in above inequality. Recall the definition of restricted $\alpha$-Hölder norm, we have

$$
A \leq d \cdot\|\nabla \boldsymbol{f}\| \cdot H_{\alpha}\left(\boldsymbol{y}-\boldsymbol{y}_{n} \mid D_{n}\right) \cdot\left|t_{i}^{n}-t_{j}^{n}\right|^{\alpha}
$$

For the second term, by mean value theorem, it is easy to have

$$
\begin{aligned}
C & \leq d^{2} \cdot\left\|\nabla^{2} \boldsymbol{f}\right\| \cdot\left(H_{\alpha}\left(\boldsymbol{y} \mid D_{n}\right)+H_{\alpha}\left(\boldsymbol{y}_{n} \mid D_{n}\right)\right) \cdot\left|t_{i}^{n}-t_{j}^{n}\right|^{\alpha} \cdot\left\|\boldsymbol{y}\left(t_{j}^{n}\right)-\boldsymbol{y}_{n}\left(t_{j}^{n}\right)\right\| \\
& \leq d^{2} \cdot\left\|\nabla^{2} \boldsymbol{f}\right\| \cdot\left(G_{1}^{*}+G_{1}\right) \cdot\left|t_{i}^{n}-t_{j}^{n}\right|^{\alpha} \cdot\left\|\boldsymbol{y}\left(t_{j}^{n}\right)-\boldsymbol{y}_{n}\left(t_{j}^{n}\right)\right\|,
\end{aligned}
$$

where the second inequality follows from Lemma 10 and 11. Note that

$$
\begin{aligned}
\left\|\boldsymbol{y}\left(t_{j}^{n}\right)-\boldsymbol{y}_{n}\left(t_{j}^{n}\right)\right\| & \leq\left\|\boldsymbol{y}(0)-\boldsymbol{y}_{n}(0)\right\|+\left\|\left(\boldsymbol{y}\left(t_{j}^{n}\right)-\boldsymbol{y}_{n}\left(t_{j}^{n}\right)\right)-\left(\boldsymbol{y}(0)-\boldsymbol{y}_{n}(0)\right)\right\| \\
& \leq\left\|\boldsymbol{y}(0)-\boldsymbol{y}_{n}(0)\right\|+H_{\alpha}\left(\boldsymbol{y}-\boldsymbol{y}_{n} \mid D_{n}\right) \cdot\left|t_{j}^{n}\right|^{\alpha}
\end{aligned}
$$

Then we have

$$
\begin{gathered}
H_{\alpha}\left(\boldsymbol{f}(\boldsymbol{y})-\boldsymbol{f}\left(\boldsymbol{y}_{n}\right) \mid D_{n}\right) \leq\left(d \cdot\|\nabla \boldsymbol{f}\|+d^{2} \cdot\left\|\nabla^{2} \boldsymbol{f}\right\| \cdot\left(G_{1}^{*}+G_{1}\right)\right) \cdot H_{\alpha}\left(\boldsymbol{y}-\boldsymbol{y}_{n} \mid D_{n}\right)+ \\
d^{2} \cdot\left\|\nabla^{2} \boldsymbol{f}\right\| \cdot\left(G_{1}^{*}+G_{1}\right) \cdot\left\|\boldsymbol{y}(0)-\boldsymbol{y}_{n}(0)\right\|,
\end{gathered}
$$

which concludes the proof of Lemma 12.

Appendix D: Applications In this section, we show how to combine our convergence rate result of the midpoint displacement construction (Theorem 1) and $\epsilon$-strong simulation algorithm with other advanced simulation techniques.

D.1. Application to Multilevel Monte Carlo We start with a brief introduction of the MLMC framework (Giles, 2008). Our objective is to estimate $\alpha=\mathbb{E}\left[g\left(B^{H}\right)\right]$, where $g$ is an $L$ Lipschitz continuous functional of the fBM path with respect to the uniform norm. Here, we define a function $g$ to be $L$-Lipschitz continuous, $L \in(0, \infty)$, if for any $x, y \in \mathcal{C}([0,1])$,

$$
|g(x)-g(y)| \leq L\|x-y\|_{\infty} .
$$

The MLMC estimator takes the following form

$$
\hat{\alpha}_{K}=\sum_{k=0}^{K} \frac{1}{r_{k}} \sum_{i=1}^{r_{k}} D_{k}(i)
$$


where $D_{k}(i)$ 's are i.i.d. copies of some properly defined level differences. For example, $D_{k}(i) \stackrel{d}{=}$ $g\left(B_{k}^{H}\right)-g\left(B_{k-1}^{H}\right)$. Assuming $g\left(B_{-1}^{H}\right)=0$, then

$$
\mathbb{E}\left[\hat{\alpha}_{K}\right]=\mathbb{E}\left[g\left(B_{K}^{H}\right)\right],
$$

which implies the bias of the estimator (43) only depends on the bias at the highest level $K$. On the other hand,

$$
\mathbb{V}\left(\hat{\alpha}_{K}\right)=\sum_{k=0}^{K} \frac{1}{r_{k}} \mathbb{V}\left(D_{k}\right)
$$

i.e. the variance depends on the variance at different levels. Thus, by using appropriate coupling to create the level differences, $D_{k}$ 's, and smartly allocating the computational budget, $r_{k}$ 's, we can achieve substantial computational cost reduction comparing to naive Monte Carlo method ${ }^{3}$. The main obstacles in applying MLMC are 1 ) how to construct $D_{k}$ 's and 2) how to calculate $\mathbb{V}\left(D_{k}\right)$ (or upper bound it).

For 1), using the midpoint displacement construction, we can set $D_{k}=g\left(B_{k}^{H}\right)-g\left(B_{k-1}^{H}\right)$.

For 2), we first note that

$$
\begin{aligned}
\mathbb{V}\left(D_{k}\right) & \leq \mathbb{E}\left[\left(g\left(B_{k}^{H}\right)-g\left(B_{k-1}^{H}\right)\right)^{2}\right] \\
& \leq L^{2} \cdot \mathbb{E}\left[\left\|B_{k}^{H}-B_{k-1}^{H}\right\|_{\infty}^{2}\right]=L^{2} \cdot \int_{0}^{\infty} \mathbb{P}\left(\left\|B_{k}^{H}-B_{k-1}^{H}\right\|_{\infty}>\sqrt{t}\right) \mathrm{d} t
\end{aligned}
$$

In Theorem 1 , by setting $\nu=0$, for any fixed $\delta \in(0, H)$, we have

$$
\mathbb{P}\left(\left\|B_{k}^{H}-B_{k-1}^{H}\right\|_{\infty}>2 \nu^{*} \ell_{k}\right) \leq 2 \exp \left\{-\left(\nu^{*}\right)^{2} \cdot 2^{2 k \delta}-2\right\} .
$$

Subsequently, let $2 \nu^{*} \ell_{k}=\sqrt{t}$ and then we obtain that

$$
\begin{aligned}
\mathbb{V}\left(D_{k}\right) & \leq 16 L^{2} \ell_{k}^{2} \cdot \int_{0}^{\infty} \nu^{*} \exp \left\{-\left(\nu^{*}\right)^{2} \cdot 2^{2 k \delta-2}\right\} \mathrm{d} \nu^{*} \\
& \leq\left(16 L^{2} \int_{0}^{\infty} \nu^{*} \exp \left\{-\left(\nu^{*}\right)^{2} \cdot 2^{2 \delta-2}\right\} \mathrm{d} \nu^{*}\right) \cdot \Delta_{k}^{2(H-\delta)}=O\left(\Delta_{k}^{2(H-\delta)}\right) .
\end{aligned}
$$

Lastly, we study the computational complexity. According to the analysis in Section 4.3, we have $\mathcal{C}\left(D_{k}\right)=O\left(\Delta_{k}^{-1} \log \left(\Delta_{k}^{-1}\right)\right)$, where the recursive Gaussian bridge based method is used. Then, for a given mean square error (MSE) bound $\epsilon^{2}$, we can set $K=C_{1} \log (1 / \epsilon)$, such that $\mathbb{E}\left[\hat{\alpha}_{K}\right]-\alpha=$ $O\left(\Delta_{K}^{H-\delta}\right)=O(\epsilon)$. We can also set $r_{k}=C_{2} \Delta_{k}^{2(H-\delta)} \epsilon^{-2} \log (1 / \epsilon)$, such that $\mathbb{V}\left(\hat{\alpha}_{K}\right)=O\left(\epsilon^{2}\right)$. With our choice of $K$ and $r_{k}$, the total computational cost of $\hat{\alpha}_{K}$ is

$$
\sum_{k=0}^{K} r_{k} \mathcal{C}\left(D_{k}\right)=O\left(\epsilon^{-2} \log (1 / \epsilon) \sum_{k=1}^{K} \Delta_{k}^{2(H-\delta)-1} \log \left(\Delta_{k}^{-1}\right)\right){ }^{4}
$$

When $2(H-\delta)>1$, the cost is $O\left(\epsilon^{-2} \log (1 / \epsilon)\right)$; otherwise the cost is $O\left(\epsilon^{-1 /(H-\delta)} \log (1 / \epsilon)^{2}\right)$. Note that the computational cost for the naive Monte Carlo estimator is $O\left(\epsilon^{-2-1 /(H-\delta)} \log (1 / \epsilon)\right)$.

${ }^{3}$ By naive Monte Carlo method, we mean generating i.i.d. copies of $g\left(B_{K}^{H}\right)$.

${ }^{4}$ Here, we treat the cost of generating the last record-breaker as a constant. This is because $N$ does not depend on $\epsilon$ and $N \leq N(\epsilon)$ almost surely as $\epsilon \rightarrow 0$. 
D.2. Application to Unbiased Estimation One important application of $\epsilon$-strong simulation algorithm is to build unbiased estimators for expectations involving functionals of the sample path (Beskos et al., 2012), or to build exact simulation algorithm for the corresponding stochastic processes at a finite collection of time points (Beskos and Roberts, 2005; Chen and Huang, 2013). Blanchet and Zhang (2017) extend the algorithm developed in Blanchet et al. (2017) to construct exact simulation algorithm for multidimensional SDEs. Pollock et al. (2016) study SDEs with jumps and provides a comprehensive discussion on $\epsilon$-strong and exact simulation. See also Glynn (2016) for an extensive review of recent development in exact simulation and unbiased estimation algorithms.

In this section, we consider the task of estimating the expectation $\mathbb{E}[g(X)]$, where $X$ denotes the sample path of a continuous time stochastic process defined on $[0,1]$ (e.g. fBM or SDEs driven by $\mathrm{fBM})$ and $g$ is a positive and $L$-Lipschitz continuous functional defined on $\mathcal{C}([0,1])$. We further assume that give any realization of $X, g(X)$ can be evaluated exactly. Our goal is to construct unbiased estimators for $\mathbb{E}[g(X)]$.

Note that unbiased estimation in this setting can be highly nontrivial, as sampling $X$ exactly is infeasible, and approximation through discretization introduces bias. We next explain how to apply the $\epsilon$-strong simulation algorithm with its tolerance-enforced property to achieve unbiased estimation as in Beskos et al. (2012).

Let $T$ be a positive random variable with a strictly positive density $p(t)$ on $[0, \infty)$, and is independent of $X$. We then define

$$
\mathcal{Z}:=\frac{1\{g(X)>T\}}{p(T)}
$$

Note that

$$
\mathbb{E}[\mathcal{Z}]=\mathbb{E}[\mathbb{E}[\mathcal{Z} \mid X]]=\mathbb{E}\left[\int_{0}^{\infty} 1\{g(X)>t\} \frac{p(t)}{p(t)}\right]=\mathbb{E}[g(X)]
$$

i.e., $\mathcal{Z}$ is an unbiased estimator of $g(X)$.

To sample $\mathcal{Z}$, we first sample $T$. Then, given $T=t$, we only need to determine whether $g(X)>t$. This can be achieved by sequentially updating $\epsilon$ and corresponding $\epsilon$-strong approximation $\hat{X}_{\epsilon}$ until we find the $\epsilon$ such that $g\left(\hat{X}_{\epsilon}\right)>t+\epsilon L$ or $g\left(\hat{X}_{\epsilon}\right)<t-\epsilon L$. Note that if $g\left(X_{\epsilon}\right)>t+\epsilon L, g(X)>t$. Likewise, if $g\left(\hat{X}_{\epsilon}\right)<t-\epsilon L, g(X)<t$. 